Florida International University FIU Digital Commons

3-24-2010

\title{
Principal Component Analysis and Assessment of Language Network Activation Patterns in Pediatric Epilepsy
}

Xiaozhen You

Florida International University, xyou001@fiu.edu

DOI: $10.25148 /$ etd.FI10041626

Follow this and additional works at: https://digitalcommons.fiu.edu/etd

\section{Recommended Citation}

You, Xiaozhen, "Principal Component Analysis and Assessment of Language Network Activation Patterns in Pediatric Epilepsy" (2010). FIU Electronic Theses and Dissertations. 176.

https://digitalcommons.fiu.edu/etd/176 


\section{FLORIDA INTERNATIONAL UNIVERSITY \\ Miami, Florida}

\section{PRINCIPAL COMPONENT ANALYSIS AND ASSESSMENT OF LANGUAGE NETWORK ACTIVATION PATTERNS IN PEDIATRIC EPILEPSY}

A dissertation submitted in partial fulfillment of the requirements for the degree of DOCTOR OF PHILOSOPHY

in

BIOMEDICAL ENGINEERING

by

Xiaozhen You

2010 
To: Dean Amir Mirmiran

College of Engineering and Computing

This dissertation, written by Xiaozhen You, and entitled Principal Component Analysis and Assessment of Language Network Activation Patterns in Pediatric Epilepsy, having been approved in respect to style and intellectual content, is referred to you for judgment.

We have read this dissertation and recommend that it be approved.

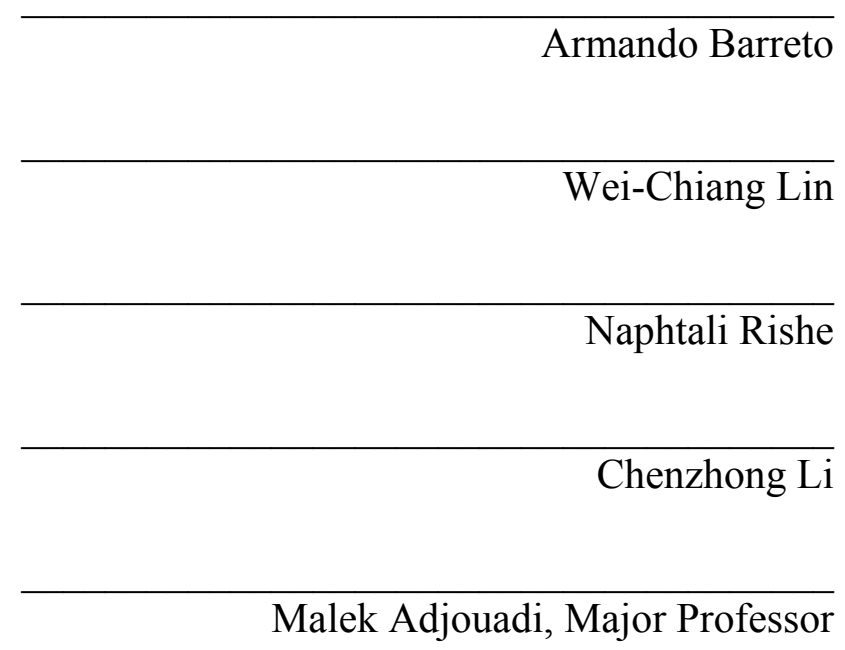

Date of Defense: March 24, 2010

The dissertation of Xiaozhen You is approved.

\begin{tabular}{r} 
Dean Amir Mirmiran \\
College of Engineering and Computing \\
\hline Interim Dean Kevin O'Shea \\
University Graduate School
\end{tabular}

Florida International University, 2010 


\section{DEDICATION}

I dedicate this dissertation to God, my best friend, my savior, Jesus Christ.

I can do all things through Jesus Christ who strengthens me.

Philippians 4:13 


\section{ACKNOWLEDGMENTS}

I thank my family though they are far away across the ocean, my spiritual family in IMIM, and my heavenly Father who has watched over me since I was in the womb. I'm very grateful to have my advisor, Dr. Malek Adjouadi. There are no words to explain my gratitude for his unconditional support, guidance, and friendship. I have great admiration and sympathy for Dr. Adjouadi who has been relentless in teaching me the meaning of being a Ph.D. student. I also want to acknowledge Dr. William Gaillard who provided the resources for this research, support in editing, and clinical information consulting. I thank Dr. Byron Bernal who gave important insights about the clinical value of this project and spent considerable time in evaluating the activation maps. I thank Magno for being a great brother in Christ, for helping me go through the process of publishing and writing the dissertation, and providing the encouragement to overcome obstacles. I also thank Melvin, Mohammed, Jin for their assistance on my research tasks. I am greatful to Ononihu, Theresa, Arlene, Steve and Ross for their encouragements during my race towards the finishing line.

Last, but definitely not least, I appreciate the support provided by the National Science Foundation under grants HRD-0833093, CNS-0426125, CNS-0520811, CNS0540592, IIS-0308155, and the Dissertation Year Fellowship provided by FIU graduate school. 


\author{
ABSTRACT OF THE DISSERTATION \\ PRINCIPAL COMPONENT ANALYSIS AND ASSESSMENT OF \\ LANGUAGE NETWORK ACTIVATION PATTERNS IN PEDIATRIC EPILEPSY \\ by
}

Xiaozhen You

Florida International University, 2010

Miami, Florida

\title{
Professor Malek Adjouadi, Major Professor
}

This dissertation establishes a novel data-driven method to identify language network activation patterns in pediatric epilepsy through the use of the Principal Component Analysis (PCA) on functional magnetic resonance imaging (fMRI). A total of 122 subjects' data sets from five different hospitals were included in the study through a web-based repository site designed here at FIU. Research was conducted to evaluate different classification and clustering techniques in identifying hidden activation patterns and their associations with meaningful clinical variables. The results were assessed through agreement analysis with the conventional methods of lateralization index (LI) and visual rating. What is unique in this approach is the new mechanism designed for projecting language network patterns in the PCA-based decisional space.

Synthetic activation maps were randomly generated from real data sets to uniquely establish nonlinear decision functions (NDF) which are then used to classify any new fMRI activation map into typical or atypical. The best nonlinear classifier was obtained on a 4D space with a complexity (nonlinearity) degree of 7 . Based on the significant association of language dominance and intensities with the top eigenvectors of 
the PCA decisional space, a new algorithm was deployed to delineate primary cluster members without intensity normalization. In this case, three distinct activations patterns (groups) were identified (averaged kappa with rating 0.65, with LI 0.76) and were characterized by the regions of: 1) the left inferior frontal Gyrus (IFG) and left superior temporal gyrus (STG), considered typical for the language task; 2) the IFG, left mesial frontal lobe, right cerebellum regions, representing a variant left dominant pattern by higher activation; and 3) the right homologues of the first pattern in Broca's and Wernicke's language areas. Interestingly, group 2 was found to reflect a different language compensation mechanism than reorganization. Its high intensity activation suggests a possible remote effect on the right hemisphere focus on traditionally leftlateralized functions.

In retrospect, this data-driven method provides new insights into mechanisms for brain compensation/reorganization and neural plasticity in pediatric epilepsy. 


\section{TABLE OF CONTENTS}

CHAPTER

PAGE

I. INTRODUCTION

1.1 Motivation

1.2 Research Problem

1.3 Significance of the Study

1.4 Structure of the Research

II. METHODOLOGY

2.1 Introduction

2.2 General Analysis Method

2.3 Data and Subjects

2.4 Software Tools

2.5 Conventional Methods

2.6 Statistical Analysis

2.7 Challenges of the Study

III. PRACTICAL MERITS OF THE PCA IN LANGUAGE FMRI ANALYSIS 31

3.1. Introduction 31

3.2. Method 32

$\begin{array}{lll}3.3 & \text { Findings } & 33\end{array}$

IV. INTEGRATING NONLIEAR CLASSIFIERS WITH PCA 40

4.1 Introduction $\quad 40$

$\begin{array}{lll}4.2 & \text { Method } & 42\end{array}$

4.3 Results 47

4.4 Discussion $\quad 59$

V. DISTANCE METHOD ON PCA-BASED DECISIONAL SPACE 63

$\begin{array}{lll}5.1 & \text { Introduction } & 63\end{array}$

$\begin{array}{lll}5.2 & \text { Method } & 65\end{array}$

$\begin{array}{lll}5.3 & \text { Results } & 67\end{array}$

$\begin{array}{lll}5.4 & \text { Discussion } & 78\end{array}$

VI. SUB-PATTERNS OF LANGUAGE NETWORK REORGANIZATION IN PEDIATRIC LOCALIZATION RELATED EPILESPY 88

$\begin{array}{lll}6.1 & \text { Introduction } & 88\end{array}$

$\begin{array}{lll}6.2 \text { Method } & 92\end{array}$

$\begin{array}{llr}6.3 & \text { Results } & 95\end{array}$

$\begin{array}{lll}6.4 & \text { Discussion } & 101\end{array}$

$\begin{array}{ll}\text { VII. CONCLUSIONS } & 106\end{array}$

LIST OF REFERENCES 111

$\begin{array}{ll}\text { VITA } & 121\end{array}$ 


\section{LIST OF TABLES}

TABLE

PAGE

Table 2. 1 Patient and subject distribution by Institution and scanner type $(*)$.......................... 18

Table 4. 1: NDF performance evaluation in percentage values ................................................. 49

Table 4. 2 : SVM performance evaluation in percentage values ................................................ 49

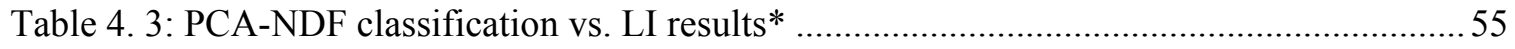

Table 4. 4: Resulting Pattern Distribution by Raters ...............................................................5

Table 4. 5: PCA Classification vs. Visual Rating Results of Three Raters....................................58

Table 5. 1: Distribution of LRE vs. control subjects in typical groups........................................ 73

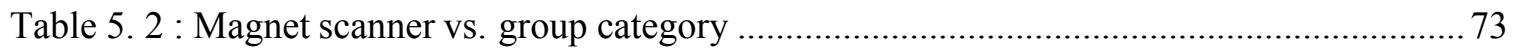

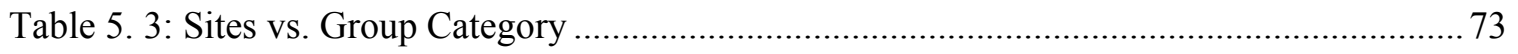

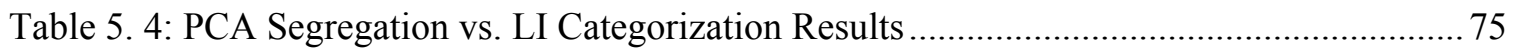

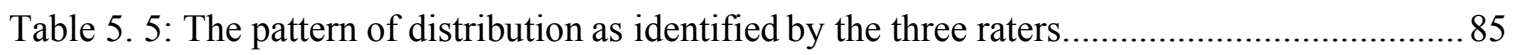

Table 5. 6: PCA Separation vs. Visual Rating results of Three Raters......................................... 86

Table 5. 7: Comparative results of second round classification using the modified Euclidean

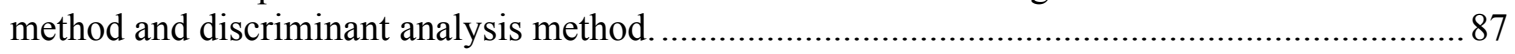

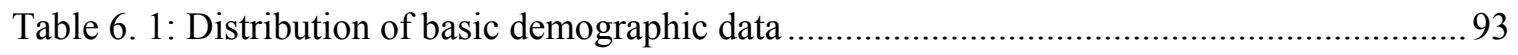

Table 6. 2: Activation location, size, peak values and commonality significance value for

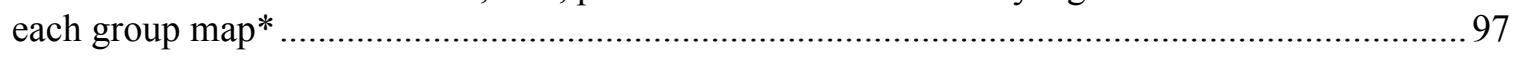

Table 6. 3: Profile of clinical factors of three groups divided by PCA method .............................. 99

Table 6. 4 : Distribution of handedness across three groups with regard to seizure focus * ....... 100 


\section{LIST OF FIGURES}

FIGURE

PAGE

Figure 2. 1: Language fMRI: Possible patterns of activation. Anterior and lateral dots represent approximate frontal language areas (Broca); posterior and lateral dots represent approximate temporal language areas (Wernicke); parasagittal small dots represent left, right or bilateral non-canonical activations. In addition, no-activation and noise (pseudo activation) are considered.

Figure 2. 2: GUI Snapshots of the Image Rating Components.

Figure 3. 1: Cumulative eigenvalues of the PCA among the 122 subjects. Note the top two eigenvectors provide $80 \%$ of the eigenvalues. 34

Figure 3. 2: The 3D representation of top two big eigenvectors $u 1$ and $u 2$ (a) Gray scale coded 2D array representation of the first eigenvector $\mathrm{u}$ 1. Slices are oriented in radiological convention: left hemisphere on the right side. (b) Representation of the second eigenvector u2.

Figure 3. 3:The zero line in the second eigenvector axis provides intuitively a rough decision line between typical $(>0)$ and atypical groups $(<0)$. Note that every data point that is on the right side of this decision line are actually left dominant $(\mathrm{LI}>0.2)$.

Figure 3. 4: The zero line in the first eigenvector axis is determined to provide a consistent decision line between higher intensity typical group $(<0)$ and atypical group $(>0)$.

Figure 3. 5: The zero line in the first eigenvector axis is determined to provide a consistent decision line between lower intensity typical group $(<0)$ and atypical group $(>0)$.

Figure 3. 6 The zero line in the first eigenvector axis is determined to provide a consistent decision line between higher intensity group $(>0)$ and lower intensity groups $(<0)$ within all the subjects that are typical.

Figure 3. 7: Visual rating distribution vs. PCA's top three eigenvectors. Black circle is rated as left dominant; red square is rated as bilateral; green star is rated as right dominant; blue diamond is rated as other (null activation or noise).

Figure 3. 8: LI distribution vs. PCA's top three eigenvectors. Black circle is left dominant as LI value indicates; red square is bilateral; green star is right dominant.

Figure 4. 1: fMRI language activation patterns in typical and atypical (Bilateral and Right) categories: Anterior and lateral dots represent expressive language areas (Broca); posterior and lateral dots represent receptive language areas (Wernicke). Note, null-activation and noise (pseudo activation) are not considered.

Figure 4. 2: Normalized projected training basis on the 3 leading eigenvectors feature space, depicting the relationships of 200 synthetic activation maps (100 typical and 100 atypical) on the 3D-feature space. 
Figure 4. 3: NDF Classifier performance for different combinations of complexity degree and the number of dimensions considered.

Figure 4. 4: Select axial cuts for illustrating mean activation patterns using NDF classifier. Brain oriented in radiological convention: left hemisphere on the right side. (a) Typical group: notice the strong left lateralization of anterior (Broca) and posterior (Wernicke) clusters. (b) Atypical group: notice the strong activation in right Broca's area....

Figure 4. 5:Selected axial cuts for mean activation patterns by SVM classifier. (a) Typical group. (b) Atypical group. Note the small difference in the patterns with respect to those shown in Figure 4.4, especially with the atypical group.

Figure 4. 6: Distribution of PCA-NDF classification results in LI of Broca's and Wernicke's areas (in intensity value). Lateralization agreement was found in 102 of 122 subjects between PCA-NDF and the LI method.

Figure 4. 7: Distribution of PCA-NDF classification results in LI of Broca's and Wernicke's areas (in voxel count or activation extent). Lateralization agreement was found in 99 of 122 subjects between PCA-NDF and the LI method.

Figure 4. 8: Distribution of PCA-NDF classification results in LI of Broca's and Wernicke's areas (averaged magnitude and extent). Lateralization agreement was found in 101 of 122 subjects between PCA-NDF and the LI method.

Figure 4. 9: Selected axial cuts for one false positive case of pattern activations where a mismatch is found between the results of the LI (magnitude) versus the results of the PCA-NDF.

Figure 4. 10: Selected axial cuts for a mismatch case where visual rating categorized as pattern 1 (strong left lateralized both in Broca's and Wernicke's areas), which is classified as typical while the PCA-NDF classified it as atypical.

Figure 5. 1: The dendrogram of the Euclidian distance matrix of the PCA suggesting there are at least three subgroups within the subjects.

Figure 5. 2: Considering only the typical subjects that satisfied the condition e $2>0$, this plot reflects the subjects' distribution based on intensity. The red squares are those subjects whose intensities are higher than the mid- point of the intensity range of the analyzed population's means; green diamonds are the ones that are lower than the mean activation intensity of these typical subjects.

Figure 5. 3: Primary clusters using the two dominant eigenvectors of the PCA. These two dominant eigenvectors are used to select three primary clusters based on the following decision rules: cluster 1: the most condensed cluster region with 32 data points; cluster 2: with ten data points; cluster 3: with five data points. The undecided region, with 75 data points, is the remaining region outside these three clusters. 
Figure 5. 4: Clusters distribution achieved using two eigenvectors. Note, data points corresponding to former undecided subjects are now assigned to one of the primary clusters based on the distance method...

Figure 5. 5:Mean activation maps for each final cluster/group. 2D array of selected axial cuts color coded for activation intensities. Higher activations are in red color. Brain oriented in radiological convention: left hemisphere on the right side. The number below the image is the slice number. (a) Mean activation map for group 1. (b) Mean activation map for group 2. The $z$ value range is higher than (a). This explains the better definition of Supplementary Motor Area (SMA). (c) Mean activation map for group 3 with an atypical right hemisphere dominant response.

Figure 5. 6: Distribution of PCA clustering results in LI of frontal and temporal language areas. "Strong right lateralized" is determined when both the LI of Broca's and Wernicke's areas are lower than -0.2. "Strong left lateralized" is determined when both the LI of Broca's and Wernicke's areas are higher than 0.2. The gray area is an atypical region including strong right lateralized and atypical bilateral. 7 out of 9 members of cluster 3 are inside the strong right dominant region.

Figure 5. 7: The linear discriminant function of PCA separation results using Euclidean Distance method.

Figure 5. 8: Classification results of undecided subjects using discriminant analysis method. Note that we circled the 6 mismatch cases between discriminant Analysis Method and our PCA-Based method.

Figure 6. 1: 2D array of selected axial cuts of the mean activation maps for each group overlaid on the MNI standard brain. Higher activations are in yellow color. Brain is oriented in radiological convention: right hemisphere on the left side. (a) Mean activation map for group 1 with strong left lateralization of anterior (Broca) and posterior (Wernicke) clusters. (b) Mean activation map for group 2 with higher mean intensity range than (a), which explains the better definition of Supplementary Motor Area (SMA). (c) Mean activation map for group 3 with an atypical right hemisphere dominant response, particularly the anterior (Broca) cluster. Different intensity threshold (90\% of the energy) was used for visualization purpose.

Figure 6. 2: Commonality significance map of each group. All three groups have the highest significance value higher than 0.8 and group 1 (a) has the least variance among the group members in the activated area, while group 3 (c) has the largest variance....

Figure 6. 3: Second level t-test comparing the mean map of group 1 to group 2. Note the high $t$ values (significant level $\mathrm{p}<0.01$ ) in the shared activated area, which is in the left IFG and MFG.

Figure 6. 4: Clinical Factor distribution among three groups. The percentage of patients in each group based on handedness, seizure focus and seizure etiology findings. Handedness was different among the three groups, and between group 1 vs. group 3, and between group $(1+2)$ vs. group 3. $(\mathrm{p}<0.0167$ Holm's sequential Bonferroni correction $)$ 


\title{
CHAPTER I
}

\author{
Introduction
}

\subsection{Motivation}

This chapter introduces first all the relevant practical issues that motivated the research foundation of this dissertation. Relevant factors for each of these issues are contextually summarized. The structure of the research and the scope of each of the chapters are then described to highlight the continuity of the tasks that have been undertaken toward a practical solution in the classification of Functional Magnetic Resonance Imaging (fMRI)-based language brain activity patterns in pediatric epilepsy.

\subsubsection{Importance of fMRI in Presurgical Evaluation for Epilepsy Population}

Research indicates that $25 \%$ of children with epilepsy do not respond to standard medications and may benefit from neurosurgery (Liegeois et al., 2006). Doctors make pre-surgery evaluations of pediatric epilepsy patients using data from MRI and EEG scans, as well as neuropsychological, neuropsychiatric, and clinical assessments. The purpose of neurosurgery in pediatric epilepsy patients is to significantly reduce the frequency of future seizures.

fMRI allows the observation of the spatio-temporal behavior of the brain activation during a given task based on the Blood-Oxygen-Level Dependent (BOLD) principle (Ogawa et al., 1990; Kwong et al., 1992; Turner et al., 1997). Through fMRI technology, it is now possible to observe brain activity patterns that reveal specific brain networks involved in the execution of a given task, related to either physical or mental 
activities (Gaillard, 2004). Precisely, noninvasive fMRI has played an important role in neurosurgical treatment for pediatric epilepsy population, since it helps identifying brain areas related to ictal or inter-ictal activity (Medina et al., 2005). In other words, fMRI has become a valuable tool for surgical planning in terms of (a) identifying functional areas for language, speech, and motor that need to be preserved, and in (b) understanding the reorganization of brain functions (e.g. language) due to tumors, structural lesions or serious brain dysfunctions such as seizures (Holloway et al., 2000). Therefore, the fMRI modality has been used extensively for brain mapping and it has become a powerful noninvasive resource for studying brain plasticity and to delimit critical brain areas to keep during resection (Binder et al., 1996; Yetkin et al., 1998).

\subsubsection{Brain Plasticity in Terms of Language Networks}

Brain plasticity is the ability of the brain to compensate for functional loss by recruiting new brain areas and reorganizing neuronal networks. Brain language networks are perhaps the most critical parts of the brain for their potential at eliciting new insight into brain plasticity.

One of the most important factors in pre-surgery evaluations is the assessment of whether language skills can be preserved after surgery. Brain language networks are known to involve the communication between canonical Brodmann Areas called Broca's and Wernicke areas (Gaillard et al., 2003). Though most left localized seizure foci and tumors affect the language behavior, it is observed that language reorganization exists during the language network's developmental stage (Liegeois et al., 2004; Szaflarski et al., 2006). It is especially interesting that, for children with left hemisphere focal brain 
injury, they rarely show speech and language impairment, which reveals the plasticity of the developing brain. Thus, pediatric epileptic patients constitute an adequate population for examining plasticity of language organization. This population will also be the one that will benefit the most once the activation patterns of language network reorganization are better understood.

\subsection{3 fMRI's Role for Language Reorganizations}

It is commonly known that language dominance is generally left hemisphere specialized. Several investigators have described reorganization of language networks from canonical areas to distinct locations, either in the same or the contra-lateral hemisphere due to the effect of structural lesions (e.g. stroke) or functional processes (e.g. epilepsy) (Liegeois et al.,2004; Szaflarski et al., 2006; Gaillard, 2004). The factors associated with the brain plasticity in terms of language networks include location of the lesion, its size, etiology, and age at seizure onset. Language lateralization for the pediatric epilepsy population cannot be reliably predicted based only on structural imaging and clinical data. However, fMRI has been recognized not only as a noninvasive, childfriendly method, but also a reliable modality to visualize the effects of lesions and seizure on language distribution in typical developing children. Therefore, it is now possible to rely on fMRI to identify patterns of language reorganizations, such as intra-hemisphere compensation, which is reorganization within the damaged hemisphere, and interhemisphere compensation, which is by recruiting atypical brain regions, or reorganize to homologous regions of the other hemisphere.

The fMRI-based approaches have been used with different paradigms to assess 
the language network behavior in terms of location and intensity of activation. The Auditory Description Decision Task (ADDT) has especially been found to reveal the subject's language area in a well-defined manner (Gaillard et al., 2004). The ADDT also serves as a good probe for receptive language areas specifically located in the middle and superior temporal gyrus, and in the inferior and middle frontal gyrus of the dominant hemisphere (Gaillard et al., 2004; Reale et al., 2007).

\subsubsection{Challenge for fMRI's General Processing Software}

The fMRIB software library (FSL) is a type of general processing software for language fMRI. It's used to perform data processing and statistical analysis to obtain the intra-subject fMRI brain activation maps. FSL often uses general linear methods (GLM) (Smith et al., 2004; Penny et al., 2007) to identify active or inactive brain regions. Moreover, on post-processing fMRI data sets, some statistical indicators are calculated, such as the Z-value. This indicator is a statistical value which defines the brain activation strength at a given voxel. For visualization purposes, a $\mathrm{Z}$ threshold value is applied to the activation map obtaining a " $\mathrm{Z}$ activation map" at a given $\mathrm{p}$ value, where $\mathrm{p}$ is the confidence probability to reject the hypothesis that the voxel inside the $\mathrm{Z}$ activation map is not activated. The activation map obtained is thus affected by the threshold parameters selected (such as the $\mathrm{Z}$ and $\mathrm{p}$ values), the coefficients chosen in the software itself, and the selected paradigm model.

The activation pattern is obtained based on statistical relationship, and there is no

golden rule in deciding whether it is related to the language network. Additionally, it takes up to 40 minutes to process one set of fMRI data. Furthermore, FSL has the 
options to process group analysis, which is called second level analysis but it requires the use of standard paradigms and acquisition parameters. This requirement is not practical when dealing with various sources of subject data. Due to these requirements, as well as to the algorithm complexity, the computational time and the memory requirements of FSL are quite demanding for processing groups of 4D fMRI data sets.

\subsubsection{Limitations of Statistical Methods and Models in fMRI}

In the last decade, there have been three major non-inferential statistical methods used for functional imaging: principal component analysis (PCA), independent component analysis (ICA) and scaled subprofile modeling (SSM) (Petersson et al., 1999). Every strategy for data analysis starts with data exploration and model selection. Model selection is a very complex process. It is impossible to cover all possible explanatory variable selection, and at the same time, make the best statistical inference for a dynamic growing population.

PCA is a non-inferential method, which means that it does not depend on a particular model selection. The PCA has been thoroughly explored in this dissertation focusing on the language activation pattern detection in a mixed growing population at the fMRI multisite at Florida International University (FIU) (http://mricate.fiu.edu/MAIN/).

Before statistical inference, PCA can point out the source of variability that might have been ignored at the beginning; it can also serve to validate that the model selected can adequately account for the systematic variability in the data after inference(Petersson et al., 1999). A voxel-by-voxel approach of PCA is proposed as an alternative to the 
region of interest (ROI) based methods, which has regionally specific hypotheses and can potentially lead to undetected effects. Our investigation also reveals that the PCA is a good mechanism for post-processing fMRI data using a large mixed population.

In contrast to PCA, the ICA spatial component (patterns) has to be not only orthogonal, but also statistically independent with few large value voxels in each component. However, the temporal component in ICA is not constrained to be orthogonal which is different from PCA's component scores ( $E$ matrix) as explained in Chapters 3. Therefore, ICA was not used as a second level analysis mechanism in this dissertation. Moreover, ICA was not used in our study, since in order to perform temporal ICA, the spatial dimensions need to be reduced so they are smaller than the time dimension. In other words, the length of the 3D activation map, aligned in one column, has to be reduced to less than the number of subjects that are included in the analysis process.

The SSM is designed for ROI-based analysis as a non-inferential multivariate method (Moeller et al., 1987). It requires the input data to be log-transformed, and then the variability of the data is decomposed into three components: global scale factor, group mean profile, and subprofile scaling factor. These component scores are similar to the ones used in PCA. SSM stands out for estimating the global effects. However, SSM was suggested to be less susceptible to artefactual decreases than other methods. No statistical model can back SSM for theoretical comparisons (Petersson et al., 1999). On the other hand, PCA may be valuable for the subspace transformation (Huber, 1985), and the investigation of the unexplained structure in the normalized residual image.

\subsubsection{Statement of Research Motives and Foundation}


Most group statistical analyses of fMRI data sets look for "commonality," under the assumption of homogeneity of the sample. However, inter-subject variance may be expected to increase in large "normal", or otherwise heterogeneous groups. In such cases, certain deviant patterns may share a common feature forming small categorical subgroups otherwise hidden within the main pooling statistical procedure. These deviant patterns may be of importance, both in normal and patient groups. The fMRI deviant language patterns may be separated by expert inspection. During this inspection, a clinical expert writes comments and rates brain activity lateralization after reviewing the activation map shown on the computer. These patterns may also be separated by means of the laterality index (LI), which is a numerical coefficient that reflects the asymmetry of distribution of activated voxels with respect to the brain midline (Wilke and Lidzba, 2007). However, the LI lacks spatial and graphical information. Expert rating may not be the optimal choice for interpreting such complex paradigms, since it's inable to scale as the number of data sets increases.

For the reasons aforementioned, there is a need for a mathematical analysis method and subsequent assessment tool for discriminating deviant spatial patterns of fMRI activation in a mixed population of control and epileptic patients. This dissertation has accomplished the design and implementation of a method as means to explore new research avenues pertaining to language network evolution on patients with epilepsy or brain lesions. This accomplishment was due to the support of a pediatric multisite consortium and a web application that provides hospitals the ability to electronically submit data consistent of 4D fMRI data sets along with clinical information on the subjects. This multisite is now increasing in the number of participants, currently 
involving 15 health institutions across America. Now as an enhancement for the multisite, a data-driven mechanism is provided to identify variant activation patterns among the uploaded population as well as statistical inference.

\subsection{Research Problem}

This dissertation focuses on developing an integrated method combining PCA, LI, clinical rating and nonlinear classifiers in order to find valid language network activation patterns in the fMRI data sets at the inter-subject level. Specific aims include: developing an easily implemented algorithm for performing standardization on the 4D fMRI data set for each subject, describing PCA-based procedures to identify sub-groups of distinct activation patterns in control and epileptic subjects, and comparing sub-groups with common clusters of activation based on the PCA algorithm results to different LI and expert clinical categorization. Additionally, the research aims at automating clinical assessment forms for fMRI language network activation patterns, in order to save human rating time and increase rating confidence. Based on the clinical info forms, statistical analysis can then be easily performed to extract population features and to examine factors that affect the language reorganization ability and to examine the correlation between certain atypical activation patterns and key factors such as seizure onset, age, and gender. The ultimate objective is to elicit new understanding of the language networks in epileptic populations by providing evidence for the language reorganization and brain plasticity in order to help doctors gauge the risk of excising a seizure focus. 


\subsection{Significance of the Study}

According to the Epilepsy Foundation, epilepsy affects more than 3 million individuals in the population of the United States alone. Epilepsy is considered the third most common neurological disorder after Alzheimer and stroke; it is as prevalent as cerebral palsy, sclerosis and Parkinson's disease, combined (Epilepsy fact Sheet: Epilepsy Foundation - http://www.epilepsyfoundation.org/about/factsfigures.cfm). Among the epileptic patients, $30 \%$ have a poor response to medications, and up to $10 \%$ are potential candidates for epilepsy surgery. With this population in mind, this study is therefore designed to investigate language networks and how they can be affected by epileptic seizures.

An automated data driven method is consequently proposed to objectively identify activation patterns and perform group analysis that will overcome the subjectivity and potential human errors by relying solely on expert rating. Once the different patterns of language organization are identified in a statistically significant population, it will be more meaningful to then assess risk for language deficits when planning for epilepsy surgery.

Furthermore, the design of the proposed method can be extended to identify variant brain activation patterns to deal with other brain abnormalities, such as Alzheimer, dementia and autism. This extension is possible because the PCA is implemented using non-normalized inter-subject intensity, as well as the way classification is performed using a unique decisional space that can manage a different number of dimensions. 


\subsection{Structure of the Research}

In structuring this dissertation, a description of the methodology used during the research process is introduced in Chapter 2. This chapter discusses the importance of the multisite repository to collect the language fMRI data of children with epilepsy, as well as normal controls from several institutions across North America. An overview of the software used to process and analyze the fMRI data sets is provided. This includes the general aspects explored in using (a) FSL for generating activation maps; (b) MATLAB for encoding certain standard correction scripts and for implementing different clustering/classification algorithms such as the PCA, nonlinear decision functions (NDF), and support vector machines (SVM); (c) STATA statistic software for carrying out poststatistical analysis. Finally, the challenges of the study, such as the limitations imposed by the conventional methods, data acquisition, data presentation, and the difficulty of making statistical inferences on clinical forms will also be addressed in this chapter.

Chapter 3 presents the results of the different experimental stages when the PCA eigensystem was explored and associated with the characteristics of fMRI language dominance considering intensities, as well as their spatial relationship. Conventional methods (LI and visual rating) are also visited. These initial developments are what lead to the new algorithms that were developed and are presented later in Chapters 4 through 6.

Chapter 4 looks into general classification techniques, introducing synthetic activation maps based on the real fMRI data pool. This chapter discusses the results of utilizing general nonlinear classifiers to classify any new fMRI activation map into two categories, typical or atypical, which is the common notion of analyzing language 
patterns. The nonlinear classifiers used in this chapter are based on a new implementation of nonlinear decision functions. The results obtained are contrasted to the wellestablished SVM for performance comparison purposes.

Chapter 5 describes the algorithm that leads to defining the initial three primary clusters found using the PCA-based decisional space and ultimately the three groups found through the inclusion of the modified Euclidean distance method. Fleiss kappa and general kappa coefficients analysis were used to assess the agreement levels between the classification of the three groups and the conventional methods (LI and visual rating).

Chapter 6 focuses on the language activation patterns that were found through the PCA-based method in Chapter 5 and their clinical relevance. It also describes the calculation of the group significance maps. It introduces the results of the patients' clinical variables analyses through STATA software. Then, clinical interpretations of the atypical language groups are made in this chapter based on the associations of the clinical variables and activation patterns in order to understand the language compensation mechanism among the epilepsy population.

Finally, Chapter 7 concludes the dissertation by providing a retrospective assessment of the merits of the proposed data-driven method within the context of a large heterogeneous population of pediatric epilepsy population, a population that is made possible through a multisite pediatric network in childhood epilepsy. 


\title{
CHAPTER II
}

\author{
Methodology
}

\subsection{Introduction}

This chapter provides a general description of the methods and essential elements that were useful for this study. It also explains the different impacts of conventional methods such as ROI-based LI and Visual Rating.

Language networks can reorganize and move from their canonical locations to distinct ones, either in the same or contra-lateral hemisphere, as a consequence of structural or functional lesions due to damaged or resected tissue. With BOLD fMRI, it becomes possible to observe brain activity patterns and seek precise anatomical localization of brain activity that represents the execution of a given task. Therefore, the first step is to project all fMRI slices onto a standard brain to achieve theoretical uniformity in analysis and assessment. This will result in viable classification measures and decision metrics. PCA is an objective and efficient multivariate analysis tool for the statistical investigation of our multidimensional data sets. As a data driven method, it creates a decisional space which is robust in clustering data sets, assessing the relevance of each dimension, and determining prime activation patterns in large multidimensional data sets. Different classification techniques, NDF and SVM were used to assess the data from different perspectives.

The multi-site fMRI repository established at Center for Advanced Techonlogy and Education (CATE) provides the source of data for pediatric epilepsy language 
network study. This consortium involves more than 15 institutions across North America. Different paradigms were stored in the consortium. Over 120 subjects who performed ADDT were processed as the population pool from this site. Each fMRI dataset was standardized with same voxel size, orientation, and dimensions. Standard MNI brain was preferred as a standard space for language activation analysis across subjects.

The PCA is performed on activation maps generated by FSL to carry out group analysis using standard MNI brain tempaltes that would delineate specific language regions. The activation map of each subject is overlaid on the standard MNI brain, thresholded for visualization purposes, and then submitted for specialists' assessment to rate the laterality in language network related areas. Furthermore, the LI is calculated for the Broca's area and the Wernicke's area separately on each subject, and a correlation between LI statistics and PCA grouping result are evaluated. The PCA group analysis results are thus validated by both the clinical rating and the LI correlations. The programming code to support preprocessing tasks was written in MATLAB.

A user-friendly GUI was created for data preprocessing to standardize each fMRI dataset, andto analyze inter-subject activation patterns. Statistical analysis was performed through STATA software. For instance, the kappa agreement test was applied to different grouping sources, either from PCA-based methods or from human rating, or LI. The Fisher exact test was also used to test the effect of different sites and scanners. ANOVA was applied to analyze the significance of age, gender, and seizure onset on atypical language activation patterns and on the ability for language reorganization. 


\subsection{General Analysis Method}

\subsubsection{PCA}

PCA as a non-inferential method has been the fundamental method for the language activation pattern detection in a mixed growing population from our fMRI multisite. Though PCA is commonly used as a dimensional reduction process, it plays an important role in multivariate statistical analysis. It can also be used as an exploratory tool to identify patterns and build models for a given multivariate population. Since PCA can identify spatio-temporal patterns, its results could potentially reveal the functional connectivity among different brain areas over time (Friston et al., 1993).

\subsubsection{General Theories and Applications of PCA}

PCA can be used in 4D fMRI at a single subject level to detect activations. Each volume is placed in a row vector, as a single multivariate observation, each column represents a voxel. Then all the rows (scans) are stacked into a data matrix X. The covariance matrix of $\mathrm{X}$, which is proportional to $\mathrm{XX}^{\prime}, \mathrm{X}^{\prime}$, being the transpose of $\mathrm{X}$, reveals the first order relationship between each pair of voxels. In order to get the PCA of XX', the step by step approach considered in this dissertation is similar to the singular value decomposition (SVD) of $\mathrm{X}$, which generates three objects: eigenvalues (singular values) that express the power or importance each eigenvector holds; component score (subject loading or small eigenvectors $\mathrm{E}$ in our 3D activation maps in second level group analysis) which shows the temporal pattern (inter subjects' variance pattern in our second level analysis); principal components (PCs), which represents the spatial patterns (eigen image or big eigenvectors $U$ in this dissertation). These three components are sorted 
according to their respective eigenvalues, such that the first component is to express the most variability across all images. $\mathrm{XX}^{\prime}$ is proportional to the sample voxel-by-voxel covariance matrix while $\mathrm{XX}^{\prime}$ is proportional to scan-by-scan (in terms of 4D fMRI PCA) or subject-by-subjects (in terms of 2nd level inter-subjects' 3D activation map analysis) covariance matrix. Correspondingly, the PCs are voxel-by-voxel eigenvectors while component scores (subject loadings) are scan-by-scan (or inter-subject) eigenvectors. They are correlated by X with the same eigenvalues. So the PCs and component scores should be interpreted concurrently. Empirical observations also suggest that clever use of key eigenvectors could be used to detect patterns that otherwise would have been unaccounted for in the data sets.

\subsubsection{Limitations of PCA}

PCA's results are sensitive to outliers and the type of preprocessing that was done

on the data sets. The PCs can change from accounting for the covariance to correlation if the image data is normalized on dividing by the standard deviation image (Joliffe and Morgan, 1992). This is also the reason why in this dissertation, normalization of intensity is later generally avoided so we can look into the effect of the original features embedded in the data sets to account for inter subject variability.

\subsubsection{Nonlinear Decision Functions}

NDF in their generalized formulation optimized with the gradient descent algorithm, as adopted in this dissertation, allows the user to select any degree of complexity or nonlinearity (r) and any number of dimensions (n) in seeking that optimal decision function with the highest accuracy in classification. The data-driven mechanism 
using NDF was found to be effective at classifying typical from atypical language networks activation patterns, even from a heterogeneous population often acquired with different acquisition parameters. The integration of PCA with the NDF classification paradigm results in a data-driven method that is both accurate and computationally appealing (within few seconds in processing time after the weights of the decision function are initially generated in the training phase). This approach could promote objective assessments of large data sets and can serve to interrogate data for a multitude of clinical variables. The main caveat is that these functions may not converge to a minimum-error stopping condition (Tou and Gonzalez, 1974; Jain et al., 2000; Duda et al., 2001) and care should be taken to avoid such pitfalls.

\subsubsection{Support Vector Machines}

The well known SVM approach maps input vectors to a higher dimensional space and seek an optimal separating hyper-plane to identify two different classes (Burges, 1998; Cristianini and Shawe-Taylor, 2000; Boser et al., 1992). SVM relies on a limited amount of kernels, such as Radial Basis Function (RBF), polynomial, etc, which don't perform well for certain problems of complex mapping, but often tend to minimize the classification risk by maximizing the inter-class marginal distances. Such an approach is used as a comparative measure to gauge the merits of the aforementioned NDFs.

\subsection{Data and Subjects}

The primary barrier to identifying factors that modulate developmental plasticity of brain networks in relation to surgical outcome lies in the difficulty of studying a sufficient 
number of patients with atypical brain activations to guide prognostic indicators of outcome (Gaillard et al., 2002, 2007; Yuan et al., 2006). The only realistic solution is to thus build a multisite in support of a research platform for the cohesive study of the human brain, bringing in synergy several hospitals and academic institutions that share the same objectives. Consequently, FIU, in partnership with hospitals with renown pediatric epilepsy programs, built a multisite repository for pediatric epilepsy data (http://mricate.fiu.edu) in order to investigate the effects of epilepsy on the brain functionality (Lahou et al., 2006). The ultimate aim is to collect data from a large population in order to identify types and subtypes of the heterogeneous expression of language networks and relate them to clinical variables such as age of brain injury, age of epilepsy onset, underlying etiology, and location of seizure focus.

Each dataset is de-identified to guarantee patient confidentiality. Procedures were followed in accordance with local institutional review board requirements; all parents gave written informed consent and children assent. Typically developing control subjects were required to be right handed and free of any current or past neurological or psychiatric disease. All patients used in this research satisfy the inclusion criteria: 1 . Patients undergoing epilepsy evaluation; 2. Patients' age between 3 and 18 years old. Subjects are excluded due to the refusal of consent or if a parent or patient requests for study termination.

This research evaluates subjects regardless of race or gender since epilepsy affects all genders and races across nations. As pointed out in Chapter 1, since epilepsy is more prevalent in children, this research focuses on pediatric patients. 


\subsubsection{Data collection}

The data collection involved a total of 133 fMRI data sets with their corresponding anatomical T1 MRIs. Null activation was observed in 11 data sets, even under modified $\mathrm{p}=0.1$ uncorrected condition, and as such, these data sets were excluded from further consideration. This lack of activation could be due to the subjects that were not following the instructions required in performing the given language task. Therefore, 122 data sets were considered valid for this study, and were distributed as follows: sixty four control and fifty eight children with LRE (see Table 2.1).

Table 2. 1 Patient and subject distribution by Institution and scanner type (*)

\begin{tabular}{|c|c|c|c|c|c|c|}
\hline Subjects & \multicolumn{2}{|l|}{ Institution } & Scanner & TR & $\begin{array}{l}\text { Voxel Size } \\
(\mathrm{mm})\end{array}$ & Num \\
\hline \multirow{5}{*}{ LRE } & $\mathrm{HSC}$ & $\begin{array}{c}\text { Hospital for Sick Children, } \\
\text { Toronto, Ca }\end{array}$ & $\begin{array}{c}\text { GE } \\
1.5 \mathrm{~T}\end{array}$ & 2 & $3.44 \times 3.44 \times 5$ & 19 \\
\cline { 2 - 7 } & $\mathrm{MCH}$ & $\begin{array}{c}\text { Miami Children's Hospital, } \\
\text { Miami,FL, USA }\end{array}$ & $\begin{array}{c}\text { Phillips Intera } \\
1.5 \mathrm{~T}\end{array}$ & 2 & $3.75 \times 3.75 \times 8$ & 10 \\
\cline { 2 - 7 } & $\mathrm{CNMC}$ & $\begin{array}{c}\text { Children's National Medical } \\
\text { Center, Washington,DC }\end{array}$ & $\begin{array}{c}\text { Siemens Trio } \\
3 \mathrm{~T}\end{array}$ & 2 & $3.44 \times 3.44 \times 4$ & 14 \\
\cline { 2 - 7 } & $\mathrm{BCCH}$ & $\begin{array}{c}\text { BC Children's Hospital, } \\
\text { Vancouver, Ca }\end{array}$ & $\begin{array}{c}\text { Siemens } \\
\text { Avanto } 1.5 \mathrm{~T}\end{array}$ & 3 & $3.44 \times 3.44 \times 3.5$ & 4 \\
\cline { 2 - 7 } & $\mathrm{CHOP}$ & $\begin{array}{c}\text { Children's Hospital of } \\
\text { Philadelphia, PA, USA }\end{array}$ & $\begin{array}{c}\text { Siemens Trio } \\
3 \mathrm{~T}\end{array}$ & 3 & $3.0 \times 3.0 \times 3.0$ & 11 \\
\hline \multirow{2}{*}{ Control } & $\mathrm{CNMC}$ & $\begin{array}{c}\text { Children's National Medical } \\
\text { Center, Washington,DC }\end{array}$ & $\begin{array}{c}\text { Siemens Trio } \\
3 \mathrm{~T}\end{array}$ & 3 & $3.0 \times 3.0 \times 3.0$ & 64 \\
\hline
\end{tabular}

\subsubsection{Image acquisition and Paradigm}

For all the participating institutions, each subject was asked to perform an auditory description decision task (a word definition task) which was designed to activate both temporal (Wernicke's area) and inferior frontal (Broca's area) cortex (Gaillard et al., 2007). The task required comprehension of a phrase, semantic recall, and a semantic 
decision. Each institution had unique acquisition parameters that were subsequently corrected and standardized. The block design paradigm consisted of $100(\mathrm{TR}=3 \mathrm{sec})$ or $150(\mathrm{TR}=2 \mathrm{sec})$ time-points, with experimental and baseline periods alternating every 30 seconds for five cycles, totaling five minutes.

During the "on" period, the participant listened to a definition of an object followed by a noun. Participants were instructed to press a button each time they judged that the description matched the noun. For instance, "a long yellow fruit is a banana" (true response) or "something you sit on is spaghetti" (Not true). Definitions occurred every three seconds. Matching pairs were pseudo-randomly distributed (70\% true responses, and 30\% foils). During baseline, the subject listened to the task definitions presented in reverse speech. The participant was instructed to press a button each time he/she heard a tone that followed the auditory string ( $70 \%$ true responses, $30 \%$ foils). The baseline was designed to control for first and second order auditory processing, attention, and motor response, while engaging the broad language processing network on an individual basis necessary for effective pre-surgical evaluation (Gaillard et al., 2007; Mbwana et al., 2009). Four age appropriate levels of difficulty were available $(4-6,7-9,10-12,>12)$.

The difficulty level was achieved by manipulating the task vocabulary based on word frequency normative data derived from reading materials (Carroll et al., 1971).

\subsubsection{Data Preprocessing}

The participating institutions provided the anatomical and fMRI data sets using distinct file formats and use different magnets. Orientation and field of view were thus corrected and standardized. Data sets were also matched into Neuroimaging Informatics 
Technology Initiative (NIFTI) format using the transversal view and radiology convention, and were finally mapped into the standard Montreal Neurological Institute (MNI) brain with $3 \times 3 \times 3(\mathrm{~mm} 3)$ voxel size and resolution of $61 \times 73 \times 61$ (axial $\times$ coronal x sagittal).

A set of scripts in MATLAB (The MathWorks, Inc.) was developed to perform the needed correction and standardization for group analysis. FSL was used to perform the pre- and post-processing required for obtaining the 3D activation maps (Jenkinson et al., 2002; Jenkinson and Smith, 2001; Rowe and Hoffmann, 2006; Woolrich et al., 2001). The data pre-processing was performed using MCFLIRT (Jenkinson et al., 2002); brain extraction using BET (Smith 2002); spatial smoothing using Gaussian kernel of FWHM $8 \mathrm{~mm}$; intra-subject mean-based intensity normalization of all volumes by the same factor; high pass temporal filtering (Gaussian- weighted least square fitting (LSF) straight line fitting, with sigma $=120.0 \mathrm{sec})$. Time- series statistical analysis was carried out using fMRIB improved linear model (FILM) with local autocorrelation correction (Woolrich et al., 2001). Post-processing was per- formed using fMRI Expert Analysis tool (FEAT) generating Z (Gaussianized T/F) statistic images thresholded using clusters determined by $Z>2.3$ and a (corrected) cluster significance threshold of $\mathrm{p}=0.05$ (Forman et al., 1995; Friston et al., 1994; Worsley et al., 1992). Registration to highresolution and standard images was carried out using FLIRT (Jenkinson et al., 2002).

\subsection{Software Tools}

We used a wide variety of software tools to support this research work. This section describes the software applications and packages that were used as fundamental 
pieces of software for data analysis, in two major steps: fMRI activation map generation and standardization; and in algorithm development.

\subsubsection{FSL}

The FSL version 4.0, developed by University of Oxford, UK, is the major software used to pre- and post- process the 4D fMRI data sets. FSL is an open source library which provided the necessary tools for analyzing fMRI data in this dissertation. These tools were used on the fMRI raw data sets to generate the activation maps for the ADDT paradigms emphasized in this dissertation. Specifically, the fMRI Expert Analysis Tool (FEAT) inside FSL was used in order to generate 3D activation maps from the 4D volumes, including the process of motion correction, alignment, filtering, model design, brain activation map results, and anatomical co-registration results (Jenkinson and Smith, 2001; Jenkinson et al., 2002; Woolrich et al., 2001). FSL was installed in LINUX environment. The procedures for processing the 4D fMRI dataset were straightforward. Moreover, the final report of operations was presented in HTML format. Therefore, FSL is user friendly for us to access the image post-processing results just by clicking on the file named: report-poststats.html. After clicking on the activation map images a new page is open to show the localization of the calculated activation clusters.

\subsubsection{Implementation Procedures}

Each dataset undergoes three processing stages in FSL: (1) Spatial processing consisting of alignment, smoothing, standardization of the 4D fMRI data sets, and temporal filtering; (2) Statistical analysis for identifying the location of the activated voxels and associated voxel clusters within the probability of confidence (p-value); (3) 
Brain co-registration for co-registering the activation map to the subject anatomical brain (native space) and also with the standard brain MNI152 (standard space). The rendered/overlaid images can be found in renderedzstat1.png.

In our second level analysis, we need to extract the folder which contains the threshold image for each individual, and correct their rotation in the case where the plane of exam is not in the standard view. The main components used in this dissertation are stats/zstat1 which contains the $Z$ statistic image $(Z=$ Gaussianised $T / F)$, and reg/example func2standard that are related to the registration of the low resolution fMRI data to the standard image.

\subsubsection{Algorithms and Models Used}

Different methodologies can be used in order to identify the voxels which are activated for the given task. One methodology is based on the pre- known homodynamic response function observed to define the model. This dissertation utilizes GLM which has been used by FSL to generate brain activation maps (Penny et al., 2007). In general, the GLM algorithm is based on modeling the data as a sum of certain factors. For fMRI raw data we can observe how each voxel's intensity varies independently for each factor in the presence of additive independent and equally distributed noise. The whole process consists in performing an analysis of variance separately for each voxel (univariate) in the time series fMRI dataset. Another approach is a model free method with no previous known condition, such as Probabilistic Independent Component Analysis (PICA) (Beckmann and Smith, 2004) and Bayesian network (Marrelecet al., 2004). However, ICA is more prone to focus more on the spatial aspects of the fMRI data and it is difficult to interpret the results, since each independent component does not have a clearly defined 
physical or physiological process as origin (Petersson et al., 1999; Guillen, et al., 2008). In contrast to PCA, the ICA spatial component (patterns) has to be orthogonal as well as statistically independent with few large value voxels in each component. However, the temporal component in ICA is not constrained to be orthogonal which differs from PCA's component scores ( $E$ matrix) as described in latter chapters. Therefore ICA has not been used for second level analysis in this dissertation.

\subsubsection{MATLAB}

MATLAB (http://www.mathworks.com/) has been used in a wide range of fields, and its easy-to-implement GUI has become a ubiquitous tool in the experimental research stage of many scientists and engineers. MATLAB is also being used to develop different tools to analyze functional Neuroimaging (Nielsen and Hansen, 2000; Yoo et al., 2004), EEG/MRI (Weber et al., 2004), especially with the advent of the enriched image processing toolbox available in MathWorks Version 6.0 or higher. Besides the script for implementations of PCA algorithms, SVM, Nonlinear Decision Functions, MATLAB scripts were also written to solve the challenges imposed by the fact that anatomical MRI data are often acquired with different views and different orientations. In this case, the motion correction and alignment algorithms from FSL will not be able to perform correctly. Therefore, MATLAB scripts for these corrections were developed before the processing with FSL is accomplished.

\subsubsection{Misc Software}

There are some software packages that were used to aid certain functions, such as format changes, Standard MNI brain template matrix, etc. 


\subsubsection{MRIcro}

The MRIcro (www.mricro.com) is a free public application with the following functions modules: view medical images, create 3D ROIs, provide Brodmann areas and anatomical templates, rendering and creating animations, overlay statistical maps or images, converting medical images formats (Rorden, 2004). It has also been used to analyze MRI and fMRI images, identifying ROIs and overlaying functional maps to anatomical MRI images. In this dissertation, due to the fact that different institutions may use different scanner and settings, fMRI scans can be converted to Analyze format from raw DICOM and MOSAIC DICOM format. Moreover, in order to generate different ROI mask for ROI-based analysis, such as LI calculation and synthetic maps generation, the Brodmann areas $(\mathrm{BA})$ were extracted from the MRIcro provided BA templates (Rorden and Brett, 2000). The templates can also be used to reduce the dimensional size of the fMRI data sets as input for PCA.

\subsubsection{Xmedcon}

Xmedcon is another public neuroimaging application for format conversion and visualization of medical images (Nolf et al., 2003). This tool can read and write most common medical image formats and 3D raw image data. Xmedcon can interface directly with AMIDE to support importing Analyze (SPM), DICOM 3.0 file formats, etc (Loening and Gambhir, 2001). It was used in this dissertation for the conversion between NIFTI and ANALYZE fMRI files.

\subsubsection{ImageJ}

The ImageJ is a Java source code-based tool that can be freely downloaded in the public domain. It is an image processing program developed at National Institute of 
Mental Health_(http://rsbweb.nih.gov/ij/features.html). It supports standard image processing functions, like display, edit, analyze, process, save and print 8-bit, 16-bit and 32-bit images, contrast, sharpening, smoothing, edge detection, filtering, etc (Abramoff et al., 2004). It was used in the early stage of this study to read and write DICOM and ANALYZE files, to evaluate the effect of reslicing the $3 \mathrm{D}$ activation maps into the standard 3mm MNI brain dimensions.

\subsection{Conventional Methods}

Investigation about language activation patterns has been mostly focused on lateralization/dominance, either through calculation of LI or clinical expert's visual rating on the $3 \mathrm{D}$ activation maps. In this dissertation, different LI calculation algorithms were tested and compared with the PCA methods; a MS-Access based visual rating system was developed to allow clinicians to rate the activation maps in a faster and easier manner.

\subsubsection{Laterality Index}

The LI is a coefficient used to indicate the asymmetry of activation patterns on the brain corresponding to a certain language task. Most previous studies of group separation by language dominance have been performed using ROI-based LI. In contrast to visual rating, the ROI measures provide absolute criteria for laterality: $\mathrm{LI} \geq 0.2$ is left; $\mathrm{LI} \leq-0.2$ is right; $|\mathrm{LI}|<0.2$ is bilateral. These criteria correspond to visual Left, Right, and Bilateral dominance. The regions employed can be hemispheric (Springer et al., 1999; Binder et al., 1996), which was the traditional notion of laterality. ROI can also be sub 
regions in the frontal and temporal lobes or any specific small region, such as specific functional Brodmann area (Ramsey et al., 2001; Gaillard et al., 2002; Spreer et al., 2002).

There are several variants of these methods: one is to count the number of voxels which exceed a set threshold in each region, another is to determine the mean $t$ score of voxels within a region, and a third is to multiply the number of activated voxels by their $\mathrm{t}$ score. However, voxel counting neglects important information of the activation intensity. In addition, voxel summation method is sensitive to statistical outliers (Price et al., 2005; Wilke and Schmithorst, 2006; Wilke and Lidzba, 2007). Recent development of LI technique is the Bootstrap method (Wilke and Schmithorst, 2006), through which the LI tends to be Gaussian distributed. First, on both hemispheres a mask is created and applied to get an activation population, and then an activation subset is defined by multiplying a fraction to the activation population. The fraction size used is chosen randomly, then the LI is calculated through predefined number of cycles and the average LI is obtained. In this dissertation, different ways of calculating LI for the same subjects were tried and compared. LI calculated through Bootstrap was used as additional information to compare results after dataset clustering using the PCA method (Wilke and Schmithorst, 2006).

Therefore, each LI was calculated using Eq.2.1 using a bootstrap method for each subject, where $\mathrm{V}$ denotes the activation magnitude or voxel count.

$$
L I=\frac{\sum V_{\text {Left }}-\sum V_{\text {Right }}}{\sum V_{\text {Left }}+\sum V_{\text {Right }}}
$$


For the sake of this work we have defined as strong right dominant for those cases in which both frontal and temporal language areas are right, left dominance if both regions are left, or one left and the other NA or bilateral, and uni-bilateral if only one area is bilateral (Fernandez et al., 2001; Gaillard et al., 2002, 2004). The specific regions were determined by Broca's area (BA 44,45,47) and Wernicke's area (BA 21,22,39), which were extracted from BA templates provided by MRIcro (Rorden and Brett, 2000).

\subsubsection{Visual Rating}

Another common way of determining language dominance is by submitting each subject's activation map to clinical experts who are blinded to subject identity and who would then score the laterality of activation in language network related areas. Each subject's activation map will be first thresholded for visualization clarity and then overlaid on top of the brain template. We developed this Access-based rating tool where all images are scored to one of a predetermined set of language network patterns representing differing combinations of activations in canonical frontal and temporal regions as given in Figure 2.1 (Gaillard et al., 2004).

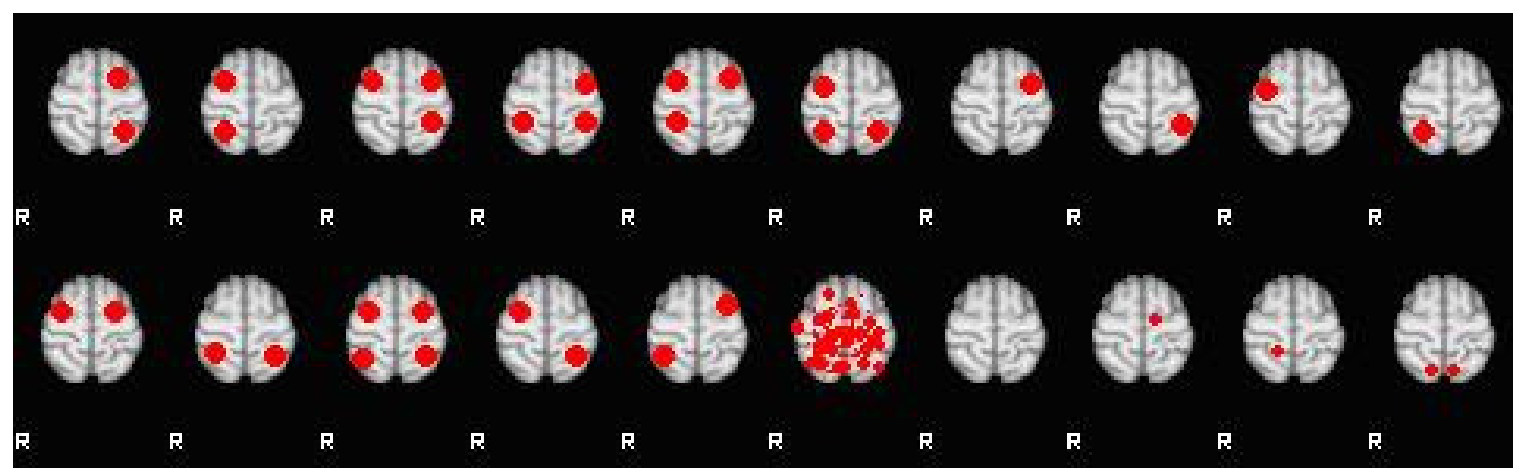

Figure 2. 1: Language fMRI: Possible patterns of activation. Anterior and lateral dots represent approximate frontal language areas (Broca); posterior and lateral dots represent approximate temporal language areas (Wernicke); parasagittal small dots represent left, right or bilateral non-canonical activations. In addition, no-activation and noise (pseudo activation) are considered. 
In addition, cases of no-activation and noise (pseudo activation) are considered as a special category. Figure 2.2 shows the rating system where there are more systematical entries with detailed regions of interest categorizations on the activation maps (Guillen, 2008). Each subject's activation map, consisting of an arrangement of 61 axial slices, was thresholded by $\mathrm{Z}>2.3$ and overlaid on top of the brain template classified with regard to overall hemispheric lateralization, language frontal lateralization (inferior frontal gyrus -IFG, Brodmann area [BA] 44,45,47, plus mid frontal gyrus -MFG, [BA] 9,46 ), and temporal language lateralization (primarily middle and superior temporal gyrus and supramarginal gyrus, BA 21,22,39). All images were scored to one of a predetermined set of language network patterns representing differing combinations of activation in canonical frontal and temporal regions. Then the identified rater provides the level of confidence in the rating. Raters can also type relevant comments on their observations. Pressing on the "done" button will save the reviewed studies.

\subsection{Statistical Analysis}

STATA is statistical data analysis software used for our statistical analysis section. Fisher exact test was applied to assess the site/scanner independence as well as the significance of association of other factors. The association of clinical factors with the group distribution was analyzed using either Fisher exact test for categorical data or ANOVA and t-test for continuous data. In our study we found more than two subgroups of activation patterns, thus pair wise comparisons of groups were performed. The Holm's sequential Bonferroni procedure was then applied to correct for the probability of a Type I error (alpha $=0.05)$. 


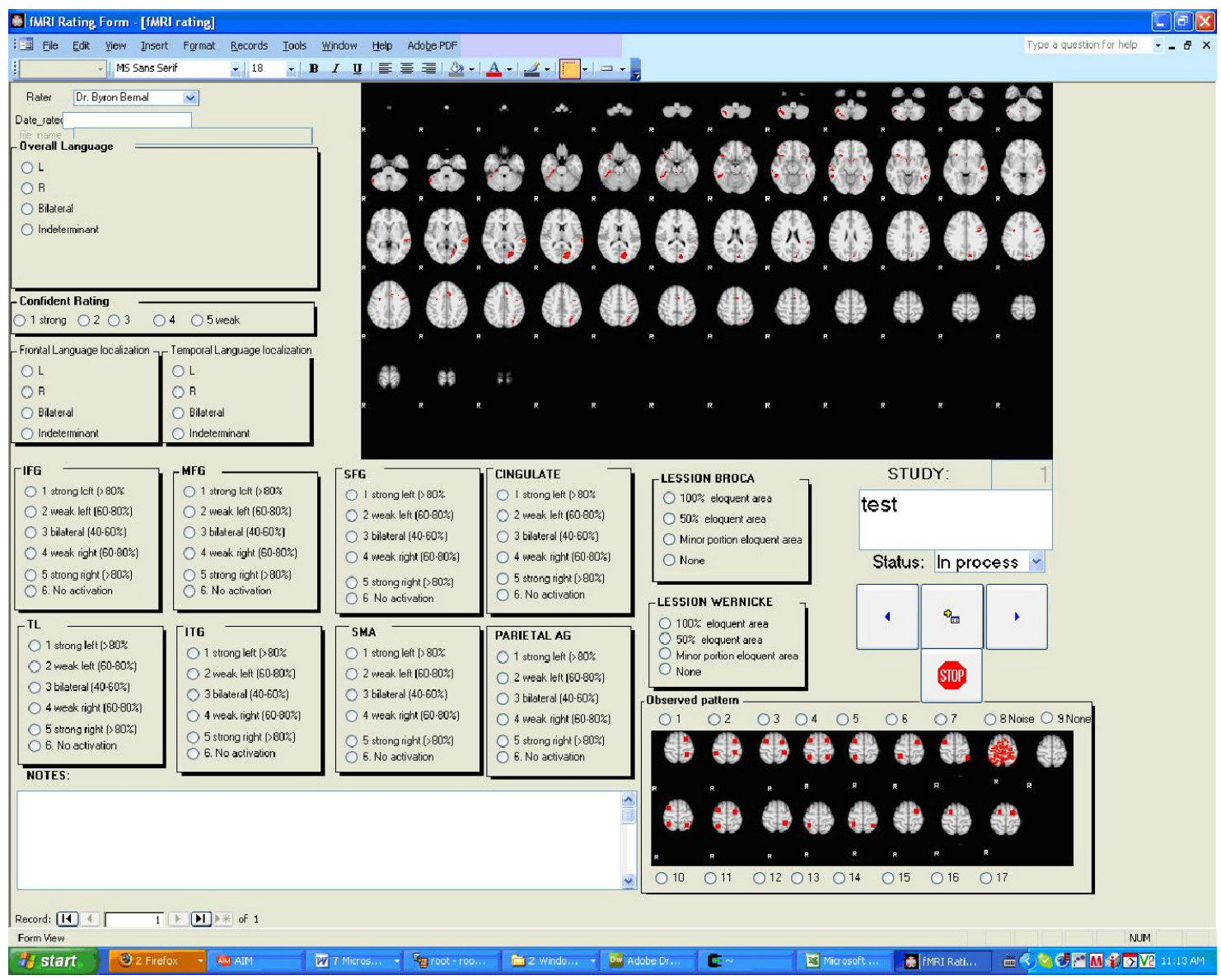

Figure 2. 2: GUI Snapshots of the Image Rating Components.

\subsection{Challenges of the Study}

\subsubsection{Acquisition Parameters Formats}

Since this is a multisite study, it's difficult to constrain all the data sets uploaded into the consortium to be of the same format and with same acquisition parameters. Different hospitals collect the anatomical and fMRI data sets using distinct file formats, plane of exam, view orientation, slicing, voxel size, repetition time (TR), and number of time points. In addition, data were obtained from either 1.5 or 3.0 Tesla magnets. Thus the orientation, slice number, voxel size and field of view have to be corrected, which is a 
time-consuming task. These issues also need inspection control over the individual data sets before we included them into the study.

\subsubsection{Clinical Information Collection and Interpretation}

Each participating institution submits the required forms in terms of three distinct types of information: (1) Medical Information, (2) Neuropsychological information, and (3) Imaging information. The Appendices in Guillen's dissertation provides the Medical Information forms suggested by the medical specialists which are important for our clinical variable analysis (Guillen, 2008). As we can be observed from the complexity of these clinical forms, it's not only time consuming but also difficult to perform data mining and interpret the correlations between the different entries. Thus we propose the multimedia Access rating tool to assess fMRI brain activation maps in a systematic way, making it more computationally efficient

\subsubsection{Limitations of the Conventional Methods}

The limitation of LI is that the actual value is affected by the defined ROI activation, threshold used, intensity range, and noise outliers (Wilke and Schmithorst, 2006; Wilke and Lidzba, 2007). For visual rating, the subjectivity is the major disadvantage since the same activation map can be categorized into different pattern or rating certainty when viewed at different time by different rater. Moreover, the processing time is another constraining factor for using this method, as the data size of the consortium keeps growing ( exceeding currently more than 100 subjects), and with the different paradigms contemplated, visual rating becomes impractical for our purposes. 


\section{CHAPTER III}

\section{Practical Merits of the PCA in Language FMRI Analysis}

\subsection{Introduction}

FMRI is capable of describing brain activity at a subject level for clinical purposes (first level analysis), such as when it's utilized for pre-surgical mapping. However, the majority of research is conducted at group level (second level analysis). Subjects are normalized using a standard anatomic atlas to ascertain the inter-subject commonality utilizing complex statistical methods. Atypical language activation pattern analysis is of significant clinical relevance in neuroscience research, especially when surgical interventions are deemed necessary. Epilepsy patient populations provide a means for validating these methods because of known heterogeneity of language dominance.

PCA is a valuable mathematical method used for the exploration and analysis of multidimensional data sets. It is further accepted that the principal components, which are orthogonal, are the linear combinations that maximize the variability of data sets (Jollife, 2002). These characteristics of PCA are incorporated in our method to transform the fMRI activation map from spatial space into eigenvector feature space.

Here, we propose a new configuration and application of the PCA for fMRI language activation pattern recognition among a heterogeneous population. The top eigenvectors are proposed to objectively automate the recognition of ROI among fMRI data sets. The potential for using eigenvectors to separate and classify fMRI language 
activation patterns is examined in this chapter. 122 subjects' fMRI activation maps from the multisite were processed. Different numbers of top eigenvectors were examined in comparison to their spatial distributions of LI and their respective visual ratings.

\subsection{Method}

The 3D activation maps generated through FSL were $\mathrm{Z}$ (Gaussianised $\mathrm{T} / \mathrm{F}$ ) statistic images with the resolution of $61 \times 73 \times 61$. As indicated earlier, there were 122 valid 3D data sets included in the PCA process.

Previous publications have reported PCA as the core analysis method for Scale Sub- profile Model (SSM), which was presented as a PCA approach for modeling regional patterns of brain function (Moeller et al., 1987; Smith et al., 2006; Alexander and Moeller, 1994). The relationship between the so-called subject loading and regional covariance pattern (eigen-image) has been widely proved (Turk and Pentland, 1991). According to the concept and merit of subject loading, we performed PCA on our 122 fMRI activation maps without masking and without intensity normalization. The following are the detailed steps in our proposed algorithm:

Step 1. Each individual's 3D dataset was transformed into a 1D dataset with $n$ voxels, where $n$ is defined by $M \times N \times L$, where $M, N$ and $L$ are the resolutions of the activation map image in the $x, y$ and $z$ axes, respectively. The whole population of subjects was organized on a $2 \mathrm{D}$ matrix $X$, where each subject contributes a specific column in the matrix. The mean value for each voxel across all subjects, which composes the mean vector $(\mathrm{m})$, was computed. 
Step 2. Each activation map was centered by subtracting the mean vector for all subjects. The covariance matrix $C_{x}$ was then calculated from Eq.3.1.

$$
C_{x}=\Psi^{T} \Psi
$$

Where $\Psi=\left[\Phi_{1} \Phi_{2} . . \Phi_{\mathrm{n}}\right]$ and each $\Phi$ is defined as $\Phi=x_{i}-m_{i}, i=1,2 \ldots k$ with $x_{i}$ being the vector containing the activation of a given subject.

Step 3. MATLAB's eigen-function was used to compute the eigenvector matrix $(E)$ of the covariance matrix $\left(C_{x}\right)$. Then the eigenvectors were sorted by the corresponding eigenvalues. Each subject was represented by a row vector $e_{. i}=\left[e_{1 i} . . e_{j i}\right]$ where $j$ corresponded to the eigenvector being used. Notice that the $E$ matrix here is equivalent to the subject loading matrix as in SSM and the $U$ matrix calculated in Eq.3.2 is equivalent to the regional covariance pattern, but instead of "regional", our $U$ is the covariance patterns of the whole $3 D$ brain region such that $\left|u_{i}\right|=1$.

$$
U=\Psi E
$$

\subsection{Findings}

The findings revealed in this chapter provide solid evidence of the merits of PCA in this research. Moreover, they also pave the foundation of the data-driven algorithm newly proposed in this dissertation, where a PCA-based decisional space is designed to identify subgroups of the mixed population in the consortium.

\subsubsection{Eigenvalues}

The process of choosing the top two eigenvectors is based on the cumulated eigenvalues of the PCA as shown in Figure 3.1. In other words, the first two 
eigenvectors carry significant information about intensity differences and overall lateralization of the activation (note that the sum of the first two most significant eigenvalues is around $80 \%$ of the total sum as seen in Figure 3.1, which means that the mean square error is $20 \%$ ).

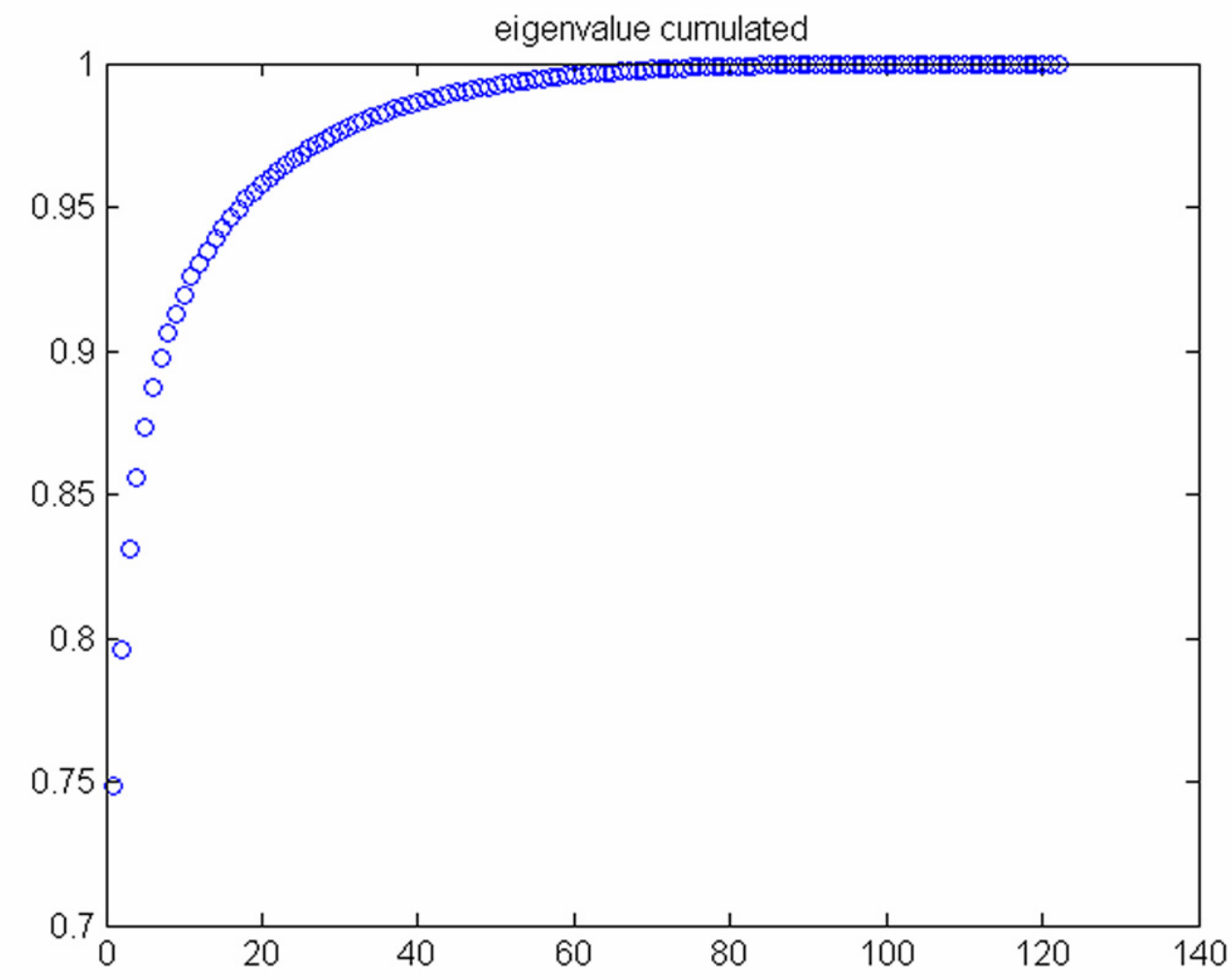

Figure 3. 1: Cumulative eigenvalues of the PCA among the 122 subjects. Note the top two eigenvectors provide $80 \%$ of the eigenvalues.

\subsubsection{Leading Eigenvectors Identifying ROI}

From the actual data of 122 subjects, scaled fMRI activation maps were aligned into 2D columns to perform PCA. As illustrated in Figure 3.2, the first 2 eigenvectors are adequate to reveal the regions of interest. Notice the strong bipolar value of anterior 
(Broca) and posterior (Wernicke) clusters. As a result, fMRI activation maps can later be masked with language areas (Broca's and Wernicke's) to reduce the image dimensions as will be demonstrated in Chapter 4.

\subsubsection{Merit of Second Eigenvector with General Language Dominance}

With all the 122 subjects considered, it was determined that the second eigenvector as the $\mathrm{x}$ axis tends to separate typical from atypical when the overall LI is used as the $\mathrm{y}$ axis (as in Figure 3.3). As we can see from this figure, the zero line in the second eigenvector axis provides intuitively a rough decision line between typical $(>0)$ and atypical groups $(<0)$. Note that every data point that is on the right side of this decision line is actually left dominant ( $\mathrm{LI}>0.2$ ). Since the mean of the second eigenvector values for those globally atypical $(\mathrm{LI}<0.2)$ is -0.0814 , and since the mean of the second eigenvector values for those globally right dominant $(\mathrm{LI}<-0.2)$ is -0.1051 , the -0.1 value (an approximate in between these two means) was chosen as a threshold criteria for primary cluster 3. These experimental evaluations will be detailed in Chapter 5 .

\subsubsection{Merit of First Eigenvector to Dominance and Intensity}

It was determined that when considering any two groups in the population, either higher intensity typical vs. atypical, or lower intensity typical vs. atypical, or even higher intensity typical vs. lower intensity typical, the zero line of the first eigenvector is sufficient to separate them as given in Figures 3.4 through 3.6. Higher activation intensity is defined as higher than the mid-point of the analyzed population's mean intensity range. On the other hand, lower activation intensity is defined as lower than the mean of the analyzed population's intensity. 


\subsubsection{Relationship of Top Eigenvectors with Visual Rating}

The visual rating distribution uses the first three eigenvectors as the axes as depicted in Figure 3.7. The figure shows a degree of disparity between the PCA and the visual rating and that the four categories are potentially separable based on their distribution using the PCA.

\subsubsection{Relationship of Top Eigenvectors with LI}

The correlation between the PCA and the LI suggest a potential for using eigenvectors to separate and classify fMRI language activation patterns as depicted in Figure 3.8 which has a smaller degree of disparity between the PCA and LI and where the 3 patterns are separable based on their distribution using the PCA.

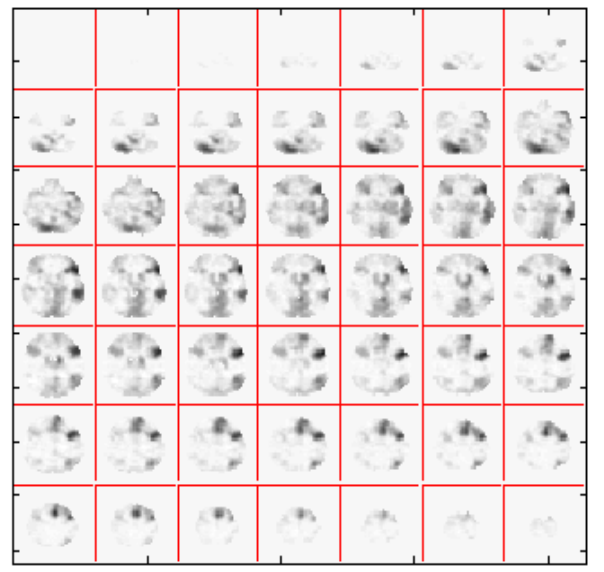

(a)
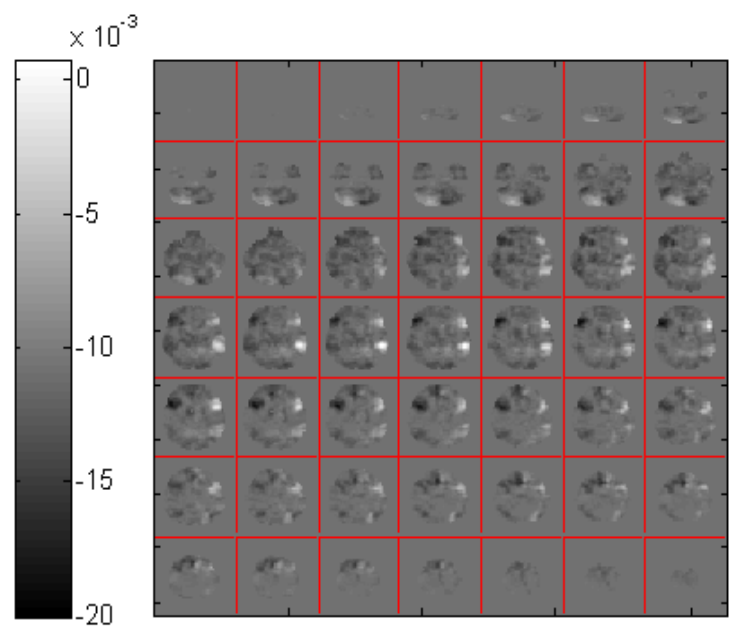

(b)

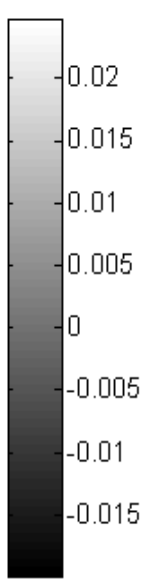

Figure 3. 2: The 3D representation of top two big eigenvectors $u_{1}$ and $u_{2}$ (a) Gray scale coded 2D array representation of the first eigenvector $\mathrm{u}_{1}$. Slices are oriented in radiological convention: left hemisphere on the right side. (b) Representation of the second eigenvector $\mathrm{u}_{2}$. 


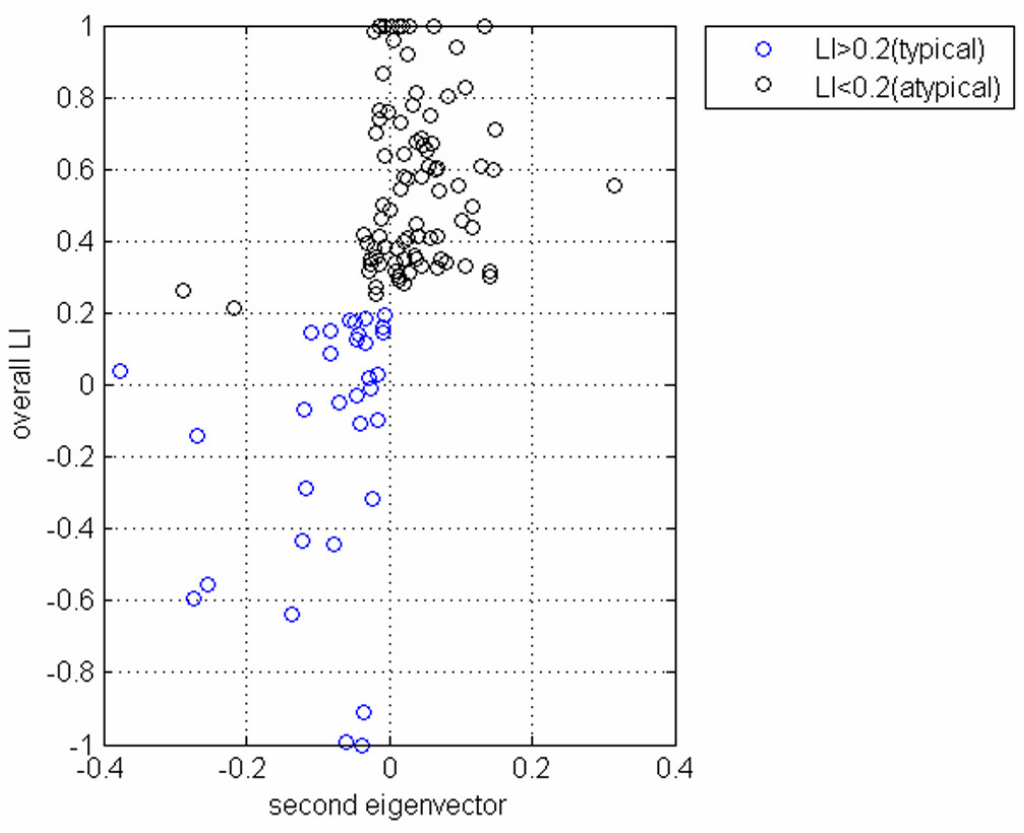

Figure 3. 3: The zero line in the second eigenvector axis provides intuitively a rough decision line between typical $(>0)$ and atypical groups $(<0)$. Note that every data point that is on the right side of this decision line are actually left dominant $(\mathrm{LI}>0.2)$.

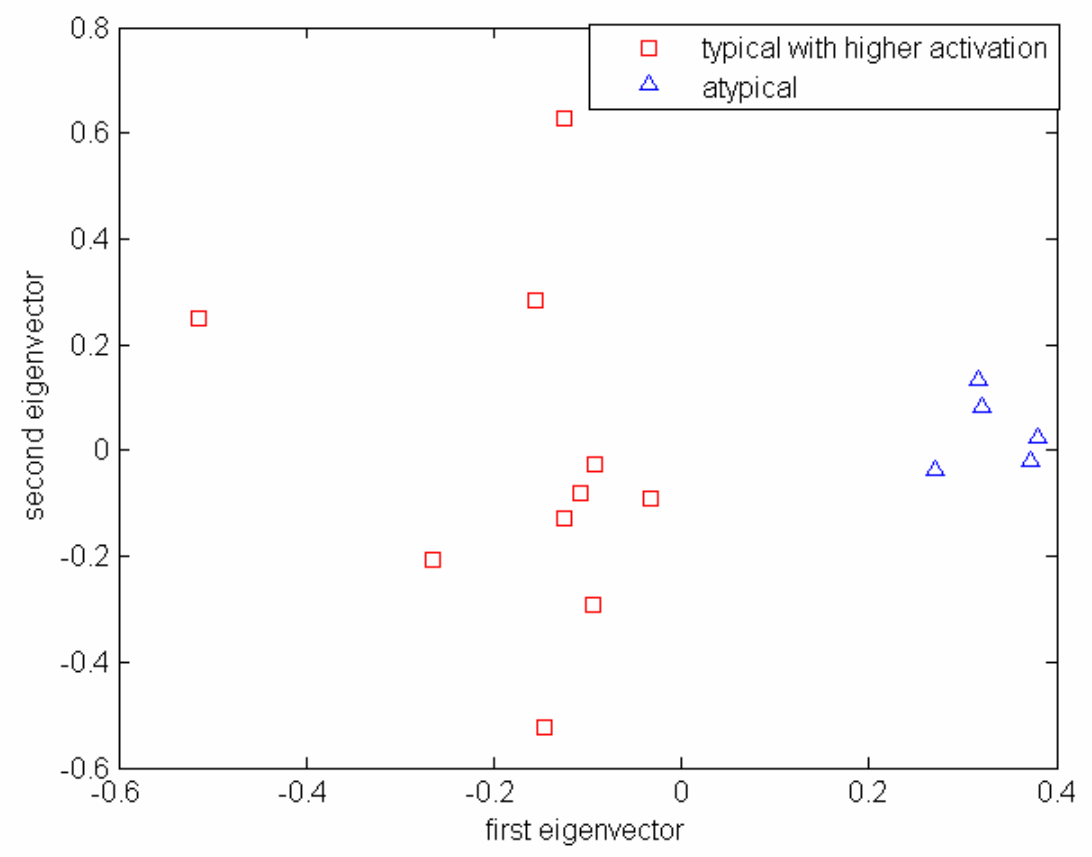

Figure 3. 4: The zero line in the first eigenvector axis is determined to provide a consistent decision line between higher intensity typical group $(<0)$ and atypical group $(>0)$. 


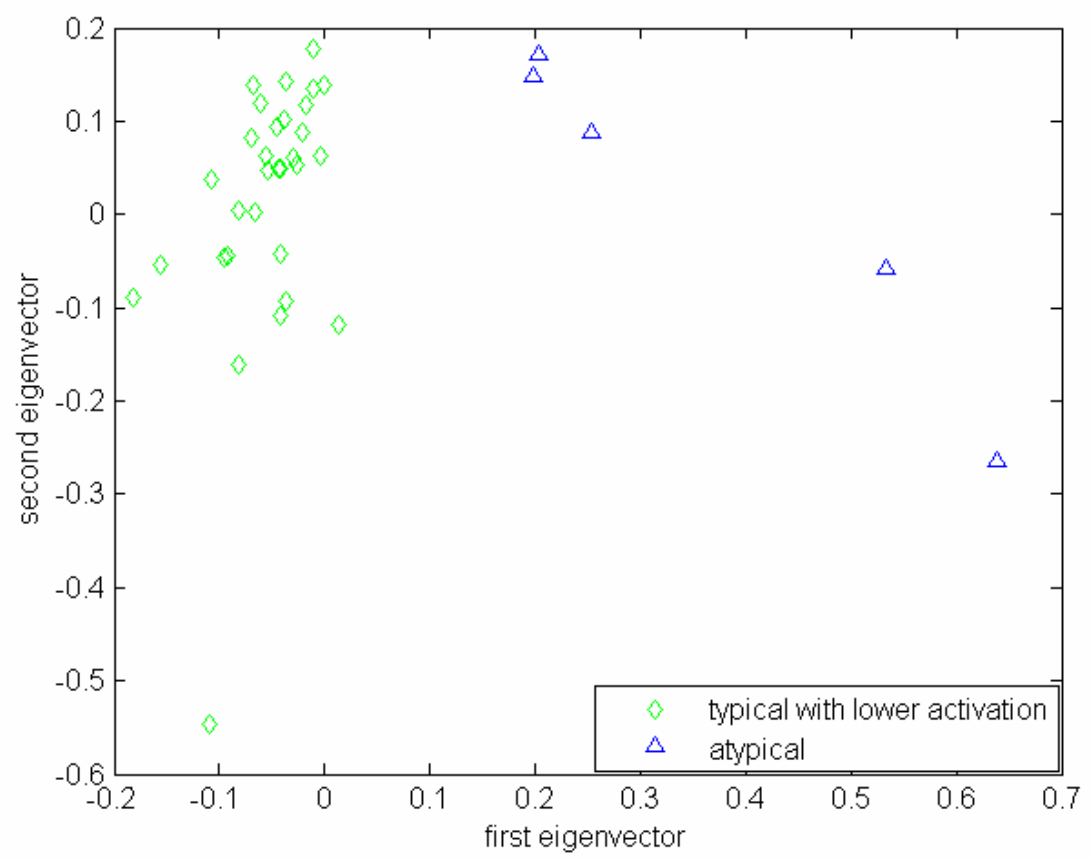

Figure 3. 5: The zero line in the first eigenvector axis is determined to provide a consistent decision line between lower intensity typical group $(<0)$ and atypical group $(>0)$.

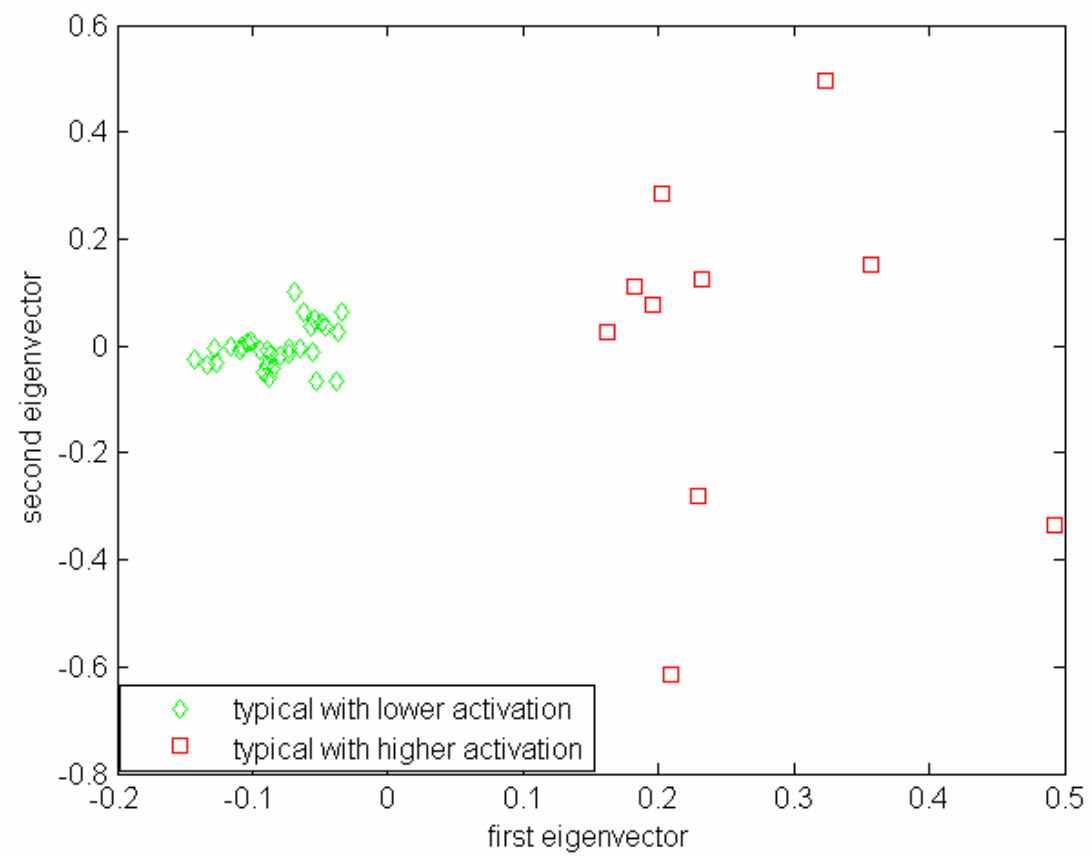

Figure 3. 6 The zero line in the first eigenvector axis is determined to provide a consistent decision line between higher intensity group $(>0)$ and lower intensity groups $(<0)$ within all the subjects that are typical. 


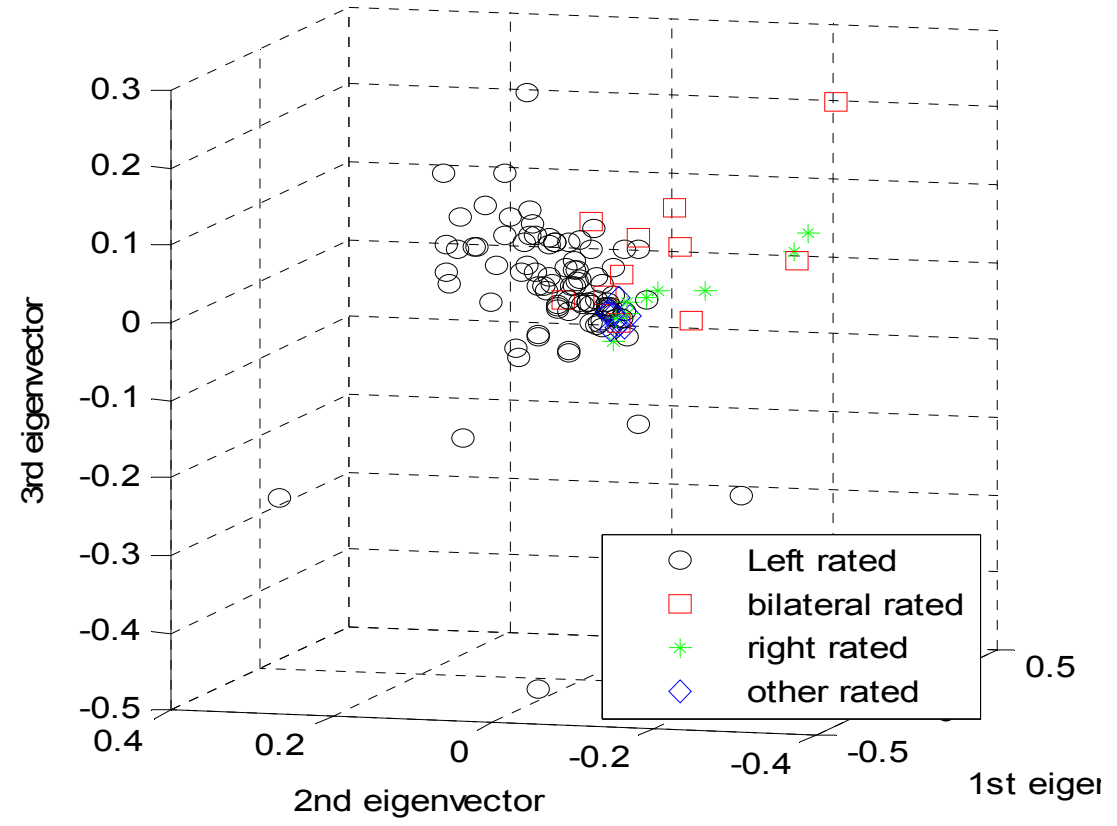

Figure 3. 7: Visual rating distribution vs. PCA's top three eigenvectors. Black circle is rated as left dominant; red square is rated as bilateral; green star is rated as right dominant; blue diamond is rated as other (null activation or noise).

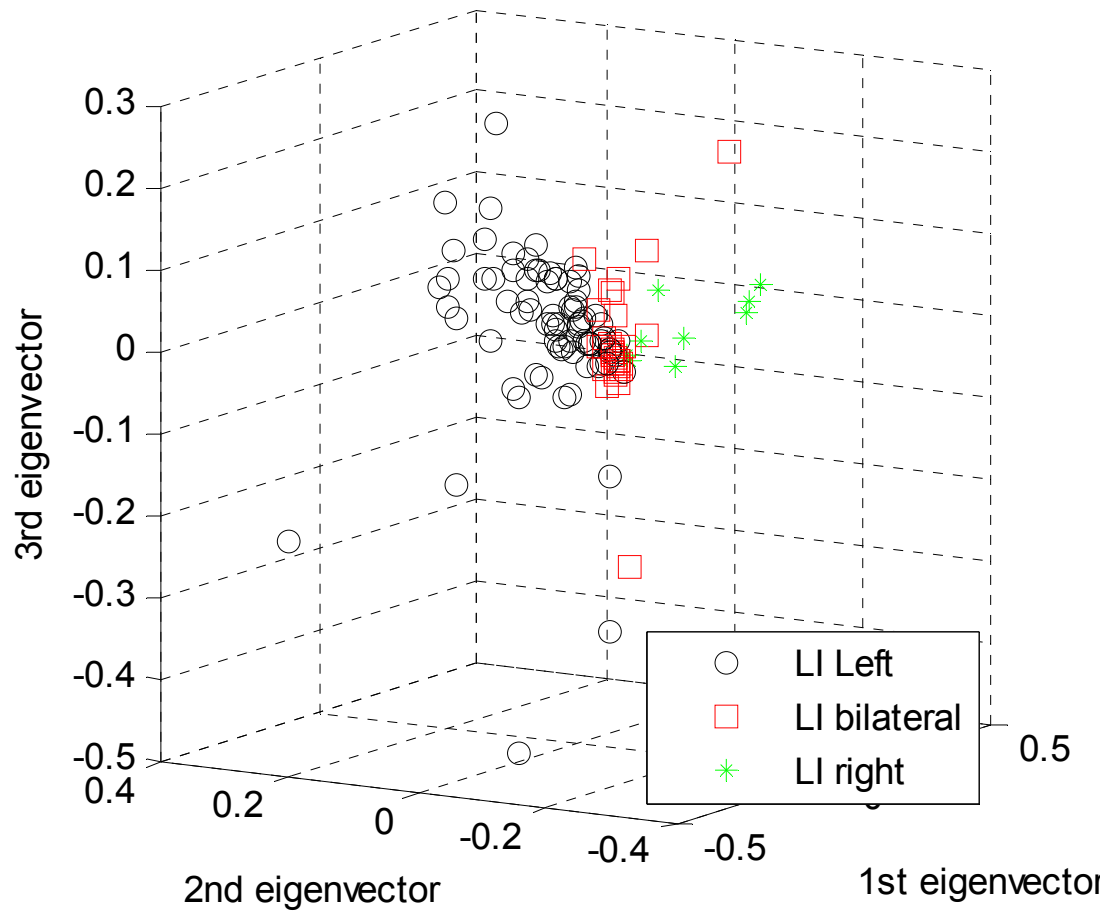

Figure 3. 8: LI distribution vs. PCA's top three eigenvectors. Black circle is left dominant as LI value indicates; red square is bilateral; green star is right dominant. 


\title{
CHAPTER IV
}

\author{
Integrating Nonliear Classifiers with PCA
}

\subsection{Introduction}

This Chapter introduces a pattern classification paradigm using nonlinear classifiers as means to automatically categorize language related fMRI activation maps into typical and atypical groups. As we have explored the merits of the top eigenvectors of PCA's decisional space in chapter 3, they can be used here as means to reduce the dimensionality of the fMRI activation maps from a classifier's perspective. The only issue is the need to train data sets for any classifier to determine the appropriate weights of the optimal decision function. There are two general obstacles/limitations of using our existing data as training data pool: (1) The sample size of existing subjects, although large, is still not adequate to have all the possible activation patterns that are suggested by the clinicians; (2) There is no golden standard to classify the existing real fMRI activation map into a category that all methods will agree. Thus in this chapter synthetic activation maps will be introduced to implement nonlinear classifiers through which the automatic process of classifying any existing and future activation maps in the consortium can be achieved.

In order to extend the practicality of these approaches, there is a need to design an automated data driven method for determining language dominance that ultimately will overcome the subjective methods that rely on visual rating and the ROI-based analysis with their inherent a priori assumptions. In a general sense, language activation patterns 
are classified into either typical or atypical categories. Atypical fMRI activation patterns were defined as those cases in which brain activation found in one or two regions is right or bilateral. Based on expert criteria, left dominance is considered typical if both regions are left, or one left and the other bilateral or non-canonical (Fernandez et al., 2001; Gaillard et al., 2002, 2004).

The essential characteristics of the PCA are thus incorporated in our method to transform the fMRI activation map from its spatial space into the eigenvector feature space. PCA is often used combining other classification techniques such as k-means (Mbwana et al., 2009), or neural networks (Samanwoy and Hojjat, 2008), to name a few. The main advantage of the PCA is the fact that it is a data-driven method as opposed to the traditional ROI-based methods that make use of different a priori empirical assumptions, and to the visual rating methods that are prone to bias. On the other hand, SVM maps input vectors to a higher dimensional space and seek an optimal separating hyperplane to identify separable classes (Boser et al., 1992; Burges, 1998; Cristianini and Shawe-Taylor, 2000).

In comparison to the generalized NDF method where the user is free to select any number of dimensions and any degree of complexity, the SVM method, although powerful in its own right, relies on a limited number of kernels. Therefore, SVM might not perform well for certain data classification problems of complex mappings, but does nonetheless attempt to minimize the misclassification risk by maximizing the inter-class marginal distances. Thus, compared to SVM, NDF used in conjunction with the gradient descent algorithm can accommodate any number of dimensions and any degree of nonlinearity (or degree of complexity), while seeking a decision space with the best 
possible accuracy in the training data sets. The main caveat is that these NDF functions may not necessarily converge to a minimum-error stopping condition (Duda et al., 2001; Jain et al., 2000), a caution that is taken into consideration in this implementation.

This chapter also presents a comparative pattern recognition study as means to automatically classify language fMRI brain activation maps from a large population into typical and atypical categories. With the multitude and diversity of the data collected came also the necessity to overcome site and scanner dependencies. To meet this need, standard data formats and processing uniformity were enforced without imposing any constraints on the hospitals providing the data. Although 122 real data sets are used, which in itself is a significant number; additional synthetic fMRI data sets are randomly generated to reinforce the classification merits of the proposed method.

\subsection{Method}

\subsubsection{Data Source - Real data sets}

The research reported in this chapter made use of the population that performed ADDT as reported in Chapter 2. Recall there are 64 control and 58 children with LRE (patient population) included in this study; each subject was asked to perform an auditory description decision task (a word definition task) which was designed to activate both temporal (Wernicke's area) and inferior frontal (Broca's area) cortex (Gaillard et al., 2007). All the 4D fMRI data sets go through the same standard preprocessing using FSL for obtaining the resulting 3D (axial x coronal $\mathrm{x}$ sagittal) activation maps, which are with the resolution of $61 \times 73 \times 61$. 


\subsubsection{Data Source - Synthetic data sets}

Synthetic activation patterns were generated according to the standard 15 patterns shown in Figure 4.1. These 15 patterns and their respective ROIs were suggested by clinical experts through extensive empirical evaluations. In accordance with the definition of typical and atypical activations, the top 5 patterns were regarded as typical (left dominant), and the rest were considered atypical (bilateral or right dominant). Based on the 122 fMRI activation maps that were considered in this study, activation in the Broca's and Wernicke's areas were extracted and saved as regional activation samples when the activated voxel number exceeded a threshold value of 400 voxels per region. For a given hemisphere, this threshold represents about $20 \%$ of the Wernicke's area, and about $15 \%$ of the Broca's area. This empirical threshold of 400 is estimated through experimental trials in order to minimize the error TYPE I, consisting in erroneously declaring presence of bilateral activation pattern on the basis of the LI when there is not, and to maximize the regional activation sample size so as to include all 15 established standard activation patterns.

With this threshold selection, the $122 \mathrm{fMRI}$ activation maps yielded the following compounded distributions: 92 with left Broca's activation, 85 with left Wernicke's activation, 60 with right Broca's activation, and 26 with right Wernicke's activation. Then these samples were randomly select and combined to generate each one of the 15 patterns as shown in Figure 4. 1. For typical category, 40 samples were created for each pattern; for atypical category, 20 patterns were created for each pattern. Total 400 synthetic data sets were generated. 


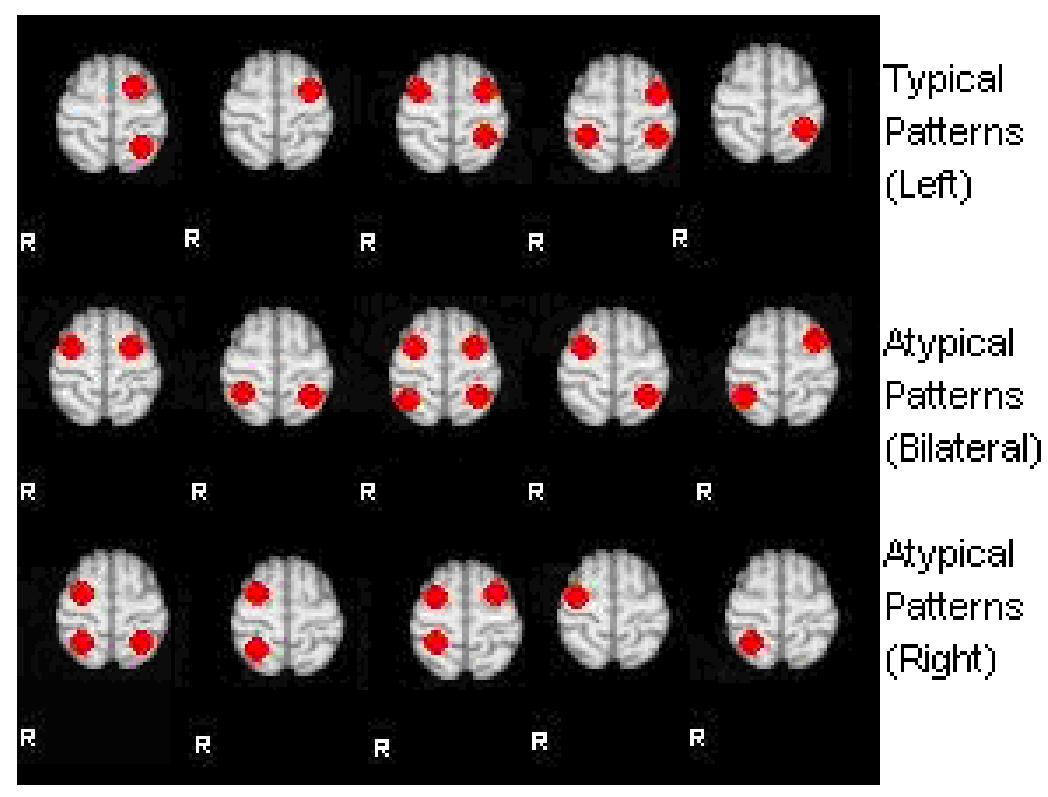

Figure 4. 1: fMRI language activation patterns in typical and atypical (Bilateral and Right) categories: Anterior and lateral dots represent expressive language areas (Broca); posterior and lateral dots represent receptive language areas (Wernicke). Note, null-activation and noise (pseudo activation) are not considered.

\subsubsection{Training and Testing Basis Generation on PCA Feature Space}

Eigen-image and projection basis in the feature space have been studied extensively with different applications in medical imaging, as well as object recognition and pattern classifications problems (Joliffe and Morgan, 1992; Turk and Pentland, 1991). With the due consideration to the concept and merit of subject loading (Alexander and Moeller, 1994), we performed the PCA on the 200 synthetic activation maps to create the feature space, and then project the rest of the synthetic maps and real subjects' data into the feature space to generate the training and testing basis. Details of the implementation steps of the PCA are provided as following after following the steps 1 through 3 as presented in Chapter 3. 
1. Project $\Psi$ into $U$ space to generate the basis as training input as in Eq.4.1.

$$
\mathrm{B}=\Psi U
$$

2. Project any new subject $\Phi_{\text {new }}$, which is the new centered $x_{\text {new }}$ $\left(\Phi_{\text {new }}=x_{\text {new }}-m_{\text {new }}\right.$ ), onto the feature space defined by the training data sets to generate the testing basis using Eq.4.2.

$$
b_{\text {new }}=\Phi_{\text {new }} U
$$

where the length or dimension of $b_{n e w}$ is determined by $j$ which represents how many leading eigenvectors were chosen.

Therefore, these bases generated on the PCA leading eigenvector feature space are served as the inputs for the nonlinear classifiers implemented in this chapter.

\subsubsection{NDF Classifier Implementation}

In order to obtain a classifier able to achieve optimal generalization, $50 \%$ of the data sets were reserved for training and the remaining $50 \%$ were used for testing. Training was performed with cross-validation to avoid memorization and increase the generalization ability of the classifiers (Tito et al., 2009).

After generating the basis for each activation map in the form of a matrix $(B)$, the NDF were then trained as classifiers on the $B$ matrix. General formulation for the NDF as introduced in (Tou and Gonzalez 1974) takes the form shown in Eq. (4.3).

$$
d^{r}(B)=\left(\sum_{p_{1}=1}^{n} \sum_{p_{2}=p_{1}}^{n} . . \sum_{p_{r}=p_{r-1}}^{n} w_{p_{1} p_{2} \ldots p_{r}} B_{p_{1}} B_{p_{2}} . . B_{p_{r}}\right)+d^{r-1}(B)
$$

where $d^{0}(B)=0$, with $\mathrm{r}$ being the complexity degree (or nonlinearity of the function) and with $n$ defining the number of dimensions in the decisional space. 
NDF classification rules for separability of two classes $\left(C_{1}\right.$ and $\left.C_{2}\right)$ are defined as follows:

$$
d^{r}(B)=\left\{\begin{array}{cc}
>0 & B \in c_{1} \\
<0 & B \in c_{2} \\
=0 & B \in\left(c_{1} \cap c_{2}\right)
\end{array}\right.
$$

In the proposed implementation, the $B$ matrix represents the basis vectors for each fMRI activation map on the PCA feature space. MATLAB code was developed to train the weight vector using the gradient descent algorithm. In this case, the stopping condition is reached when an optimal accuracy is obtained in the training phase, which sets the final update of the weight $(w)$ vector used in the decision function of Eq.4.3.

\subsubsection{SVM Classifier Implementation}

In this case, MATLAB's bioinformatics toolbox (2007) was used to train the projected basis, and generate the support vectors as classifiers. Different SVM kernels with different parameters were tried from 1D to 5D feature spaces for performance comparisons with the NDF-based method. The following standard kernels were considered: polynomial, Gaussian Radial Basis, linear, and Multilayer Perceptron (MLP).

\subsubsection{Classifier Results agreement analysis}

In order to corroborate the automatic PCA grouping results, each subject's activation map was visually rated by three experts blinded to subject identity in order to score the laterality of activation in language network related areas.

In order to show the relationship between the LI as given in Eq.2.1 of Chapter 2, this chapter will explore three different way of calculating LI. Each LI was calculated based on magnitude of activation ( $\mathrm{Z}$ score) as well as voxel count using a bootstrap 
method for each subject (Wilke and Schmithorst, 2006). The averaged LI is determined by Eq.4.5 in order to take both activation magnitude and extent (voxel count) into consideration.

$$
L I_{\text {avg }}=\left(L I_{\text {exent }}+L I_{\text {_magnitude }}\right) / 2
$$

In order to show the correlation between on one hand the experts' grouping and the PCA clustering results, and on the other hand between the LI measurements and the PCA clustering results, the members of the resulting clusters were statistically analyzed using quantity inter-rater agreement with Kappa coefficient (Viera and Garrett, 2005).

\subsection{Results}

\subsubsection{Classification Inputs}

The PCA process drastically reduced the input dimensions for the classifier. It was found that the eigenvalues of 5 leading eigenvectors represented $90 \%$ of the entire processed information for the 200 synthetic data sets used in the training phase. The projected training basis on the 3 leading eigenvectors is presented in Figure 4.2 for visualization purposes. This figure also shows that it's not optimal to train the classifier with bases projected just on these 3 leading dimensions.

\subsubsection{NDF Classifier performance}

Different complexity orders with different dimensions were trained and tested. Figure 4.3 and Table 4.1 provide the performance results with benchmarks to evaluate the complexity degree effect on the $1 \mathrm{D}$ to $5 \mathrm{D}$ feature space. A decision function with four 
dimensions ( $n=4)$ and with a complexity (nonlinearity) degree of $r=7$ was found to yield the best performance on the synthetic testing data with an accuracy of $96.00 \%$, a sensitivity of $97.00 \%$, a specificity of $95.00 \%$, and a precision of $95.10 \%$.

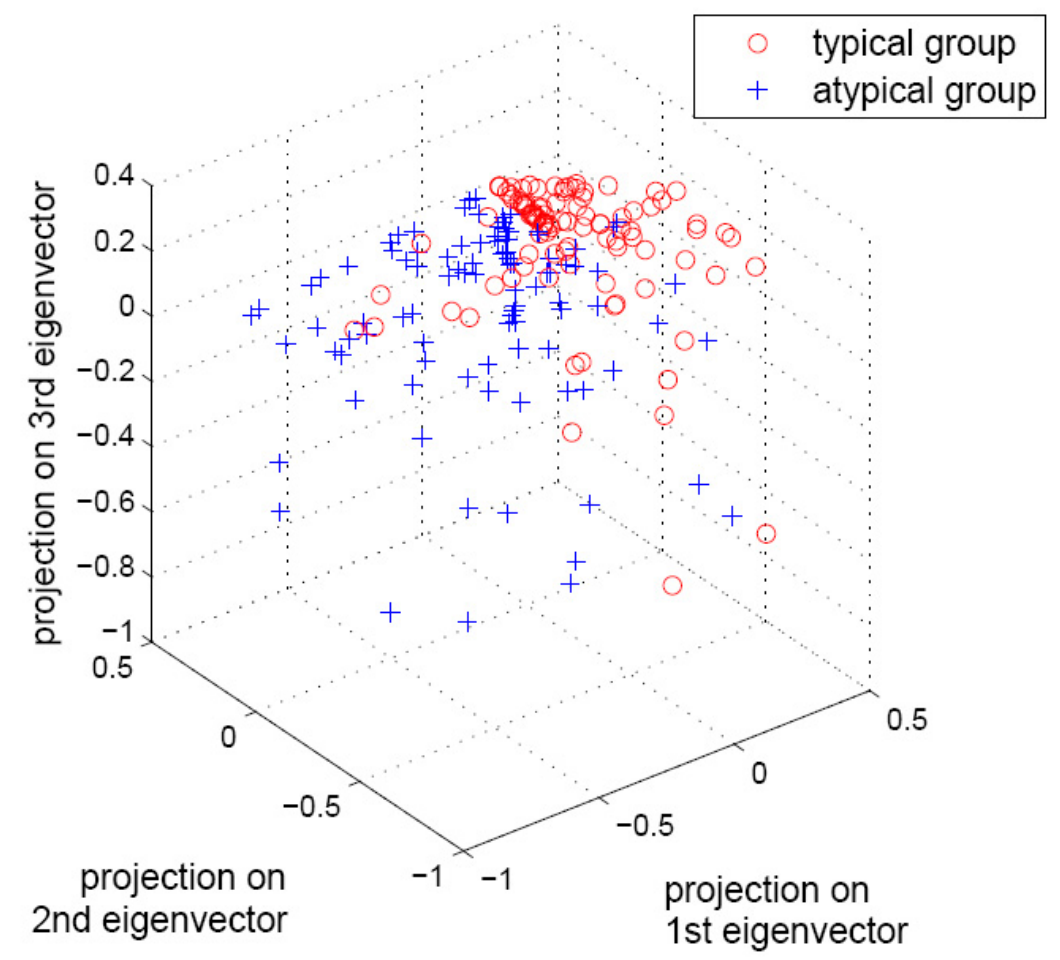

Figure 4. 2: Normalized projected training basis on the 3 leading eigenvectors feature space, depicting the relationships of 200 synthetic activation maps (100 typical and 100 atypical) on the 3D-feature space.

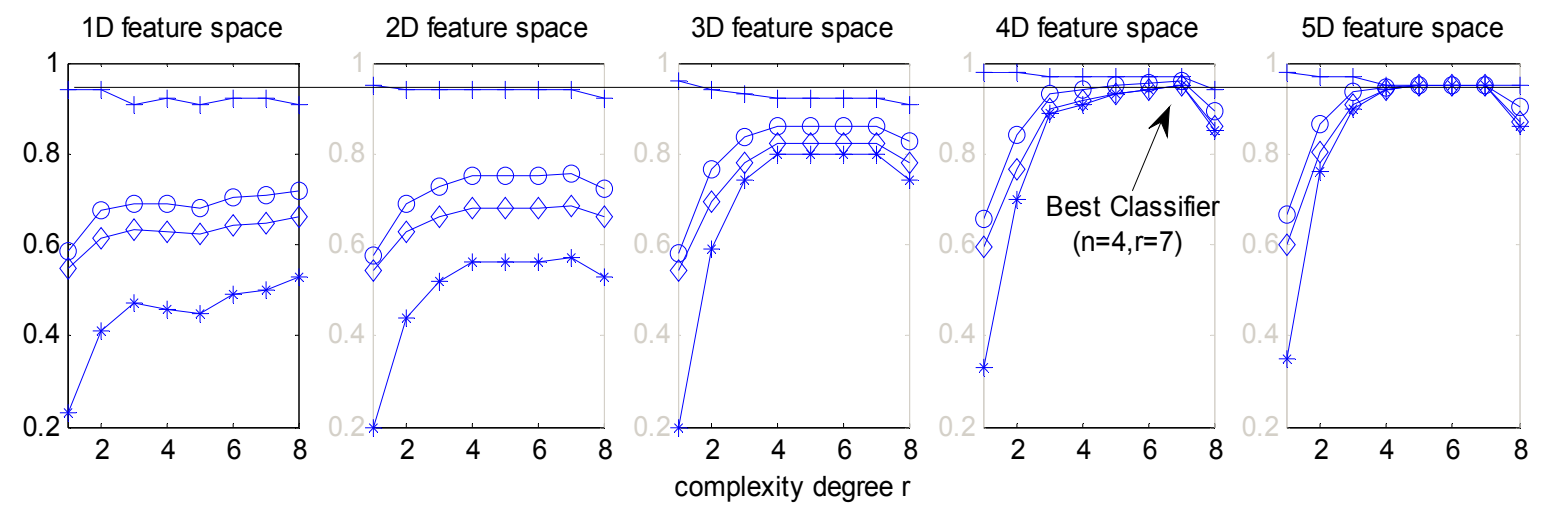

Figure 4. 3: NDF Classifier performance for different combinations of complexity degree and the number of dimensions considered. 
Table 4. 1: NDF performance evaluation in percentage values

\begin{tabular}{|c|c|c|c|c|c|}
\hline Dim. & $\begin{array}{c}\text { Best Complexity } \\
\text { Degree }(r)\end{array}$ & Acc. & Sens. & Spec. & Prec. \\
\hline 1D & 8th order & 72.00 & 91.00 & 53.00 & 65.94 \\
\hline 2D & 7 th order & 75.50 & 94.00 & 57.00 & 68.61 \\
\hline 3D & $4,5,6$, or 7 th order & 86.00 & 92.00 & 80.00 & 95.14 \\
\hline 4D & 7 th order & 96.00 & 97.00 & 95.00 & 95.10 \\
\hline 5D & 5,6, or 7 th order & 95.00 & 95.00 & 95.00 & 95.00 \\
\hline
\end{tabular}

(Acc.: Accuracy, Sens.: Sensitivity, Spec.: Specificity, Prec.: Precision)

\subsubsection{SVM Classifier performance}

In the implementation of SVM, just like with the NDF method, different kernels were tested under different dimensional spaces. For comparative purposes, the best performance for each dimension is given in Table 4.2. Polynomial kernels of SVM were found to experience more difficulties when handling higher degrees of complexity in 4D space.

Table 4. 2 : SVM performance evaluation in percentage values

\begin{tabular}{|c|c|c|c|c|c|}
\hline Dim. & Best Kernel & Acc. & Sens. & Spec. & Prec. \\
\hline 1D & $\begin{array}{c}\text { Polynomial } \\
\text { 3rd order }\end{array}$ & 78.00 & 76.92 & 80.00 & 76.00 \\
\hline 2D & $\begin{array}{c}\text { Polynomial } \\
\text { 4th order }\end{array}$ & 82.00 & 81.00 & 83.00 & 82.65 \\
\hline 3D & $\begin{array}{c}\text { Polynomial } \\
\text { 5th order }\end{array}$ & 90.00 & 91.00 & 83.00 & 84.26 \\
\hline 4D & $\begin{array}{c}\text { Polynomial } \\
\text { 3rd order }\end{array}$ & 93.50 & 94.00 & 93.00 & 93.07 \\
\hline 5D & RBF & 91.00 & 93.00 & 89.00 & 89.42 \\
\hline
\end{tabular}

(Dim.: Dimension, RBF: Radial Basis Function, Acc.: Accuracy, Sens.: Sensitivity, Spec.: Specificity, Prec.: Precision) 


\subsubsection{LRE language fMRI classification}

After applying the optimal 4D NDF classifier with complexity degree of 7 on the actual language-area-masked data sets, the 122 real data sets were separated into 2 groups. The distinct activation patterns of these 2 groups are as depicted in Figure 4.4 using selected axial cuts. According to the classification results, $34 \%$ of the LRE population shows atypical activation. For comparative purposes, the classification results obtained on applying the optimal SVM classifier are shown in Figure 4.5. These results were achieved utilizing polynomial kernel of third order on the 4D feature space. Note the differences in the activation patterns, especially in the atypical cases. Although both NDF and SVM recognize the atypical right dominant activations in right Broca's area, they differ in the right Wernicke's area.

\subsubsection{PCA-NDF vs. LI classification Results}

The cluster distribution plots associated with the LI of Broca's and Wernicke's areas are shown in Figures 4.6 through 4.8. Each figure depicts different aspects of LI calculation depending on the activation feature used. These figures illustrate that in the atypical group, LRE subjects were more associated with atypical right-brain dominant activation. Though LI will not strictly agree with the specific classification results of the PCA-NDF, LI agree well with PCA-NDF in finding the most atypical activation pattern (which is right dominant in the cases considered). In addition, the extent of classification mismatches of the combined PCA-NDF versus the LI of Broca's and Wernicke's areas in terms of false positives and false negatives are illustrated in these aforementioned three figures. 
Classification of the 122 subjects according to both methods: PCA-NDF and LI are provided in Table 4.3. This categorization assumes a classification into typical and atypical activation. The strength of agreement for voxel count LI is considered "moderate" (Kappa $=0$. 559; confidence interval (CI): [0.397, 0.721]), while for magnitude is "good" (Kappa $=0.614$; CI: [0.459, 0.769]).

Figure 4.9 shows the activation patterns of the case where LI classified the pattern as typical (through magnitude, extent and averaged value) and the PCA-NDF classified the same pattern as atypical. We believe that this false positive classification is largely due to the non-canonical activations and the presence of some activation on the right hemisphere, so it is still a deviant from the typical group. However the strong maxima activation on the left hemisphere tends to force the LI into the range as typical. Therefore, this needs further examination for a more appropriate investigation through invasive means, such as intracarotid amobarbital test (IAT).

(a)
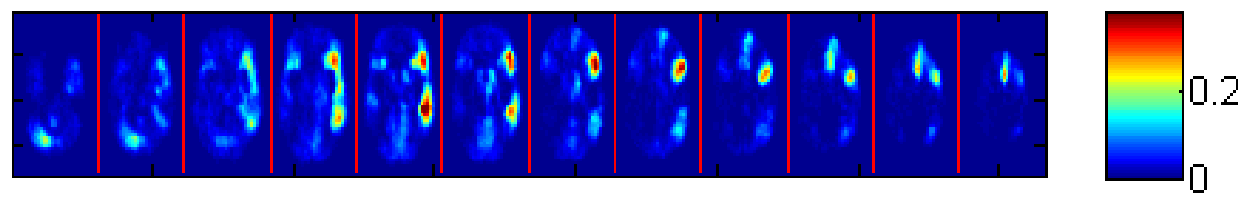

(b)
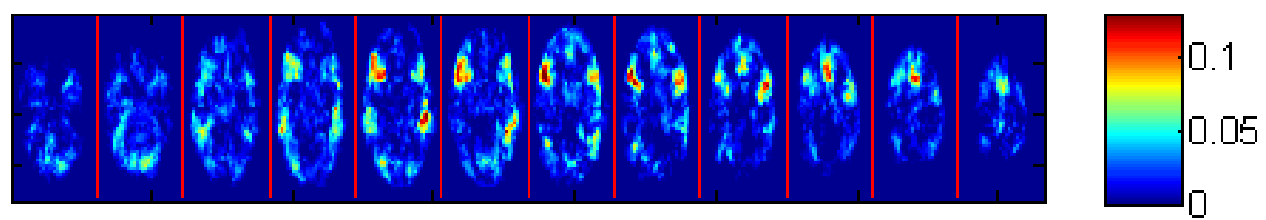

Figure 4. 4: Select axial cuts for illustrating mean activation patterns using NDF classifier. Brain oriented in radiological convention: left hemisphere on the right side. (a) Typical group: notice the strong left lateralization of anterior (Broca) and posterior (Wernicke) clusters. (b) Atypical group: notice the strong activation in right Broca's area 
(a)
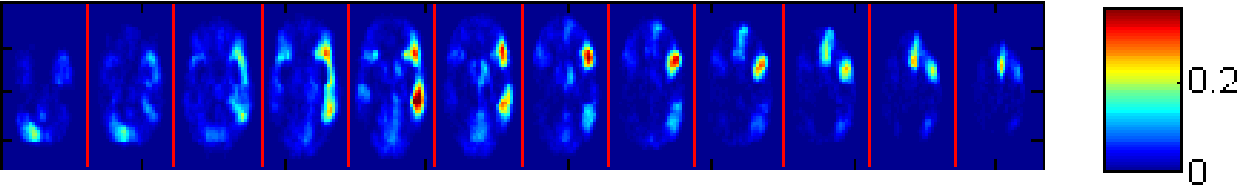

(b)
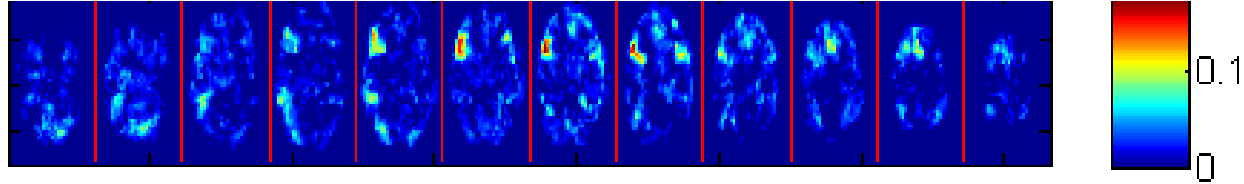

Figure 4. 5:Selected axial cuts for mean activation patterns by SVM classifier. (a) Typical group. (b) Atypical group. Note the small difference in the patterns with respect to those shown in Figure 4.4, especially with the atypical group.

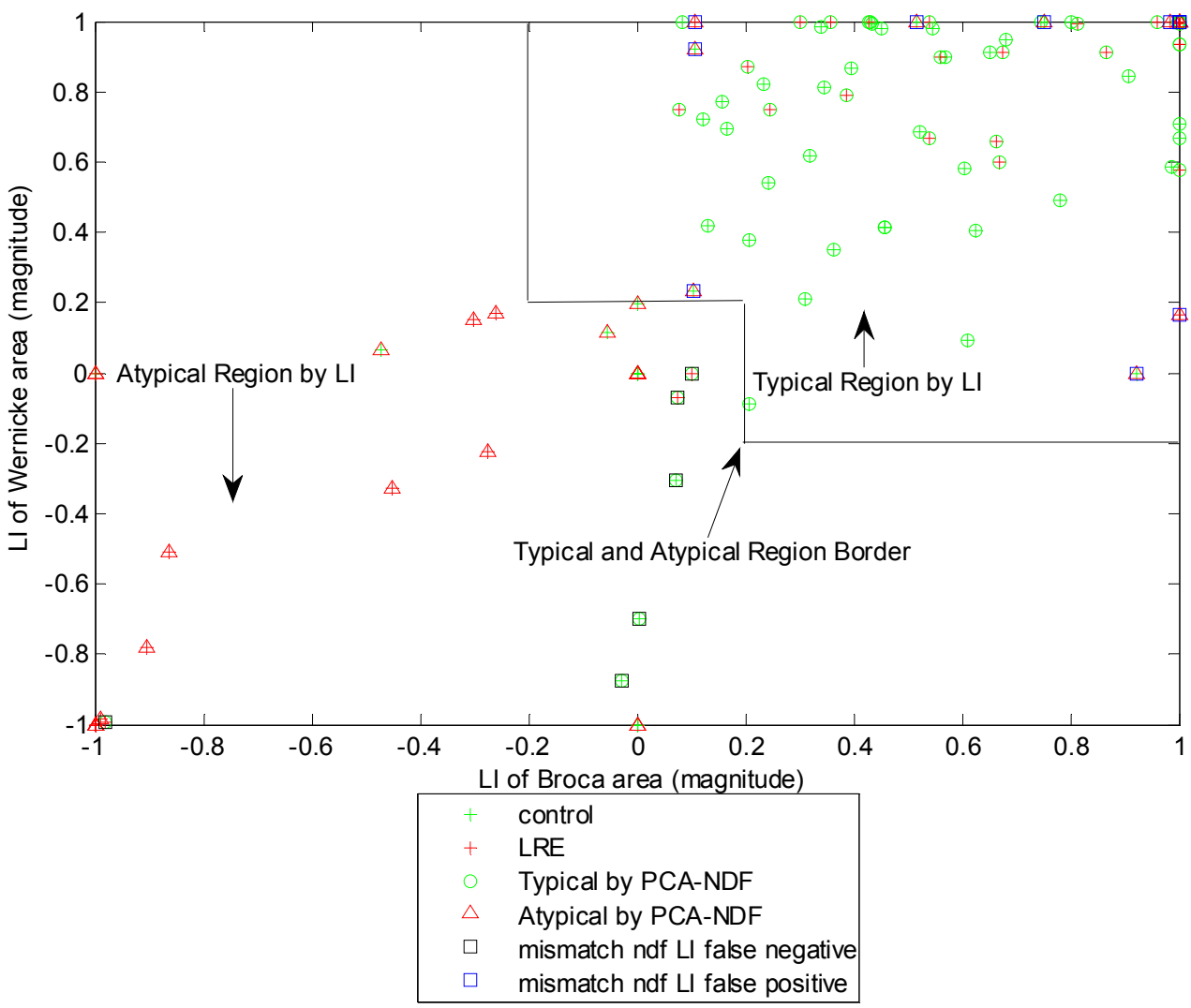

Figure 4. 6: Distribution of PCA-NDF classification results in LI of Broca's and Wernicke's areas (in intensity value). Lateralization agreement was found in 102 of 122 subjects between PCA-NDF and the LI method. 


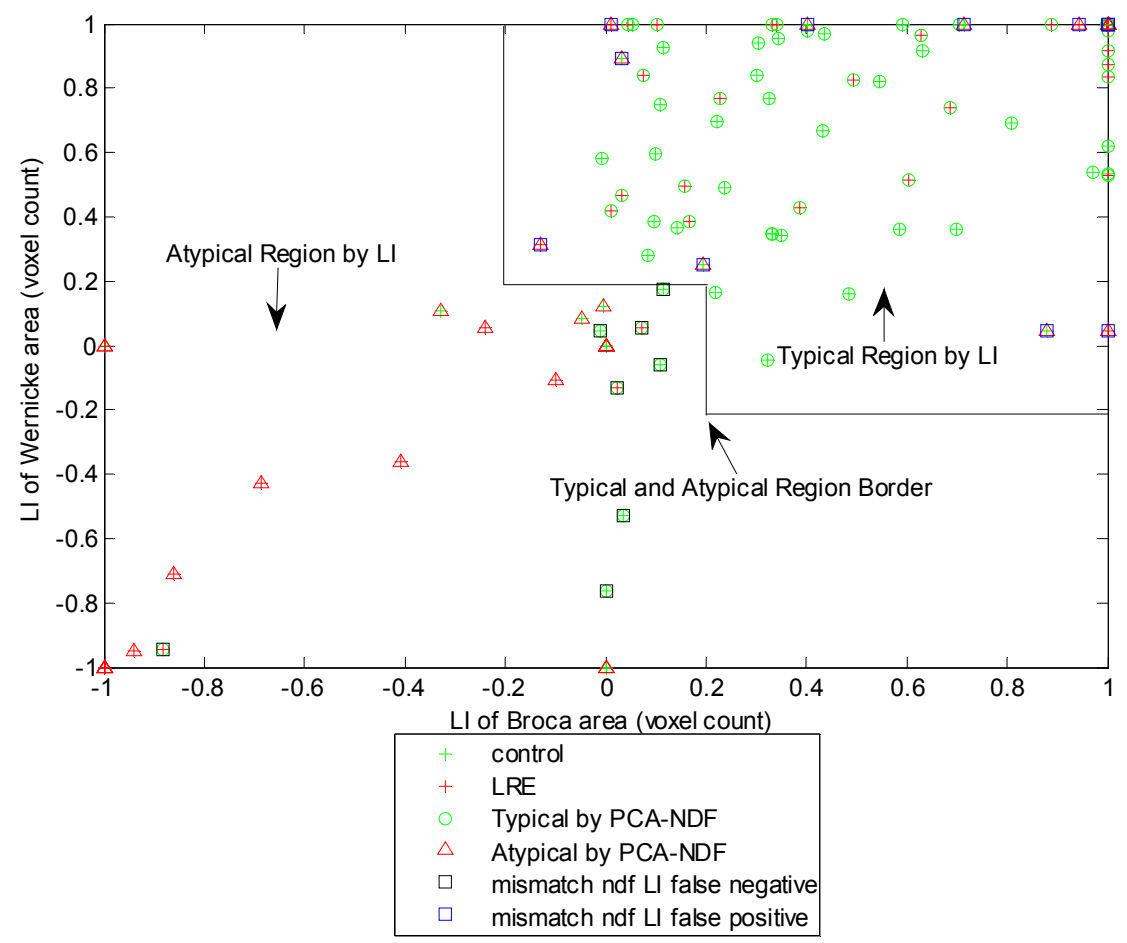

Figure 4. 7: Distribution of PCA-NDF classification results in LI of Broca's and Wernicke's areas (in voxel count or activation extent). Lateralization agreement was found in 99 of 122 subjects between PCANDF and the LI method.

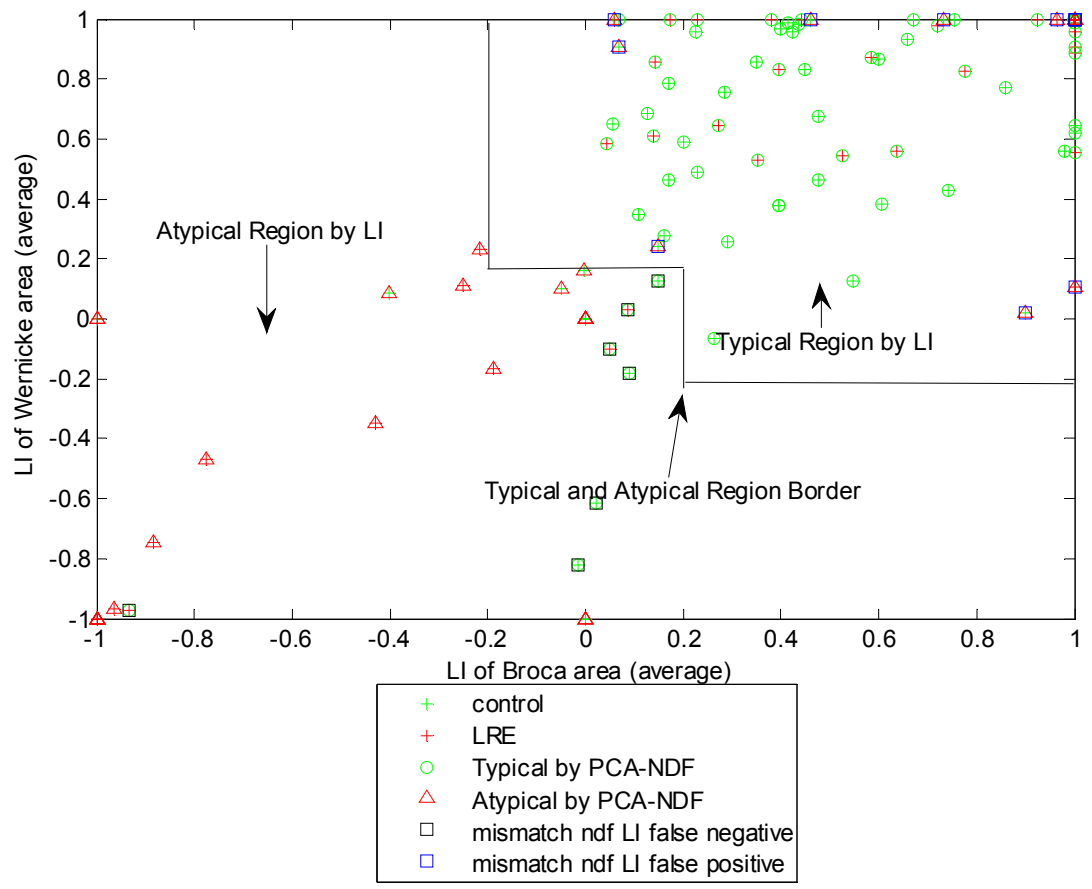

Figure 4. 8: Distribution of PCA-NDF classification results in LI of Broca's and Wernicke's areas (averaged magnitude and extent). Lateralization agreement was found in 101 of 122 subjects between PCA-NDF and the LI method. 
Table 4. 3: PCA-NDF classification vs. LI results*

\begin{tabular}{|c|c|c|c|}
\hline LI_mag & Typical & Atypical & Total \\
PCA-NDF & & & \\
\hline Typical & 75 & 6 & 81 \\
\hline Atypical & 14 & 27 & 41 \\
\hline Total & 89 & 33 & 122 \\
\hline LI_ext & Typical & Atypical & Total \\
\hline PCA-NDF & & & \\
\hline Typical & 73 & 8 & 81 \\
\hline Atypical & 15 & 26 & 41 \\
\hline Total & 88 & 34 & 122 \\
\hline LI_avg & Typical & Atypical & Total \\
\hline PCA-NDF & & & \\
\hline Typical & 74 & 7 & 81 \\
\hline Atypical & 14 & 27 & 41 \\
\hline Total & 88 & 34 & 122 \\
\hline
\end{tabular}

*Classification of typical and atypical activation comparing the PCA-NDF and the LI categorization by three different variants of LI calculation methods: activation intensity (magnitude), activation extent (voxel count), and their averaged value. Lateralization agreement was found from 99 to 102 out of 122 subjects. The strength of the agreements ranged 'moderate' to 'good'. (Kappa coefficient $(\kappa)=0.559$ to $0.614 ; \mathrm{p}=$ $0.05)$.

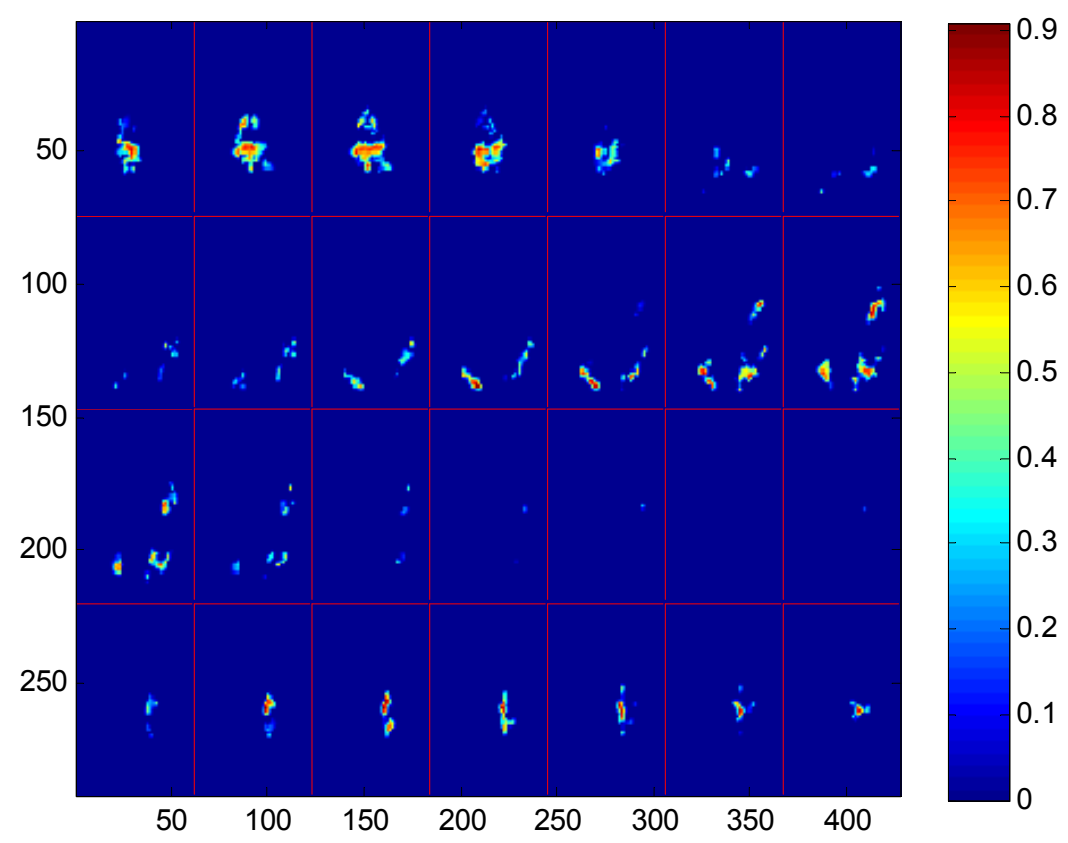

Figure 4. 9: Selected axial cuts for one false positive case of pattern activations where a mismatch is found between the results of the LI (magnitude) versus the results of the PCA-NDF. 


\subsubsection{PCA-NDF vs. Visual Rating Categorization Results}

The visual rating results are shown in Table 4.4, in this table each subject was assigned to one of the general patterns (Figure 4.1) by the three raters. The inter-rater agreement among the three raters was very high (Fleiss Kappa: $0.8132, \mathrm{p}=0.05$ ) with their distribution shown in table 4.4, though we can still see some discrepancies among raters. Their agreement with PCA-NDF results vs. raters' language dominance categorization is provided in Table 4.5. Note that the non-canonical activation was depicted here as no activation. The agreements were found significant when comparing each rater's result to PCA-NDF's (Kappa ranged from 0.52 to 0.67 ). Note that there are a few patterns shown in Figure 4.1 which were not included in table 4.4, since those activation patterns were not found.

Table 4.5 shows a concordance of $80 \%$ to $86 \%$ between the visual rating and the automatic group separation obtained by the PCA-NDF method. The strength of the agreement for classification of typical and atypical activation comparing the PCA and the visual rating categorization ( $80 \%$ to $86 \%$ ) is considered "moderate" to "good" (Kappa 0.52 to $0.67 ; \mathrm{p}=0.05)$.

Figure 4.10 shows one activation pattern that was classified as typical using visual rating while the PCA-NDF classified that same pattern as atypical. We believe this disagreement is largely due to some activation at both Broca's and Wernicke's areas on the right hemisphere, therefore it is still a deviant from the typical group. This opens the possibilities of a type II error on the visual rating method, and PCA-NDF can serve as an alternative method to corroborate existing LI and visual rating classification results. Again, the reader should be aware that none of these methods can be considered as a standard golden rule, but the only ultimate confirmation is one that would be given either by invasive means such as the IAT or through electrical cortical stimulation (ECS). 
Table 4. 4: Resulting Pattern Distribution by Raters

\begin{tabular}{|c|c|c|c|c|c|c|c|c|c|}
\hline \multirow[b]{2}{*}{ Typical } & \multicolumn{3}{|c|}{ Rater 1} & \multicolumn{3}{|c|}{ Rater 2} & \multicolumn{3}{|c|}{ Rater 3} \\
\hline & Control & LRE & Total & Control & LRE & Total & Control & LRE & Total \\
\hline & 37 & 30 & 67 & 38 & 31 & 69 & 39 & 33 & 72 \\
\hline & 1 & 3 & 4 & 2 & 4 & 6 & 1 & 4 & 5 \\
\hline & 5 & 3 & 8 & 7 & 2 & 9 & 4 & 3 & 7 \\
\hline & 6 & 5 & 11 & 4 & 5 & 9 & 4 & 4 & 8 \\
\hline & 1 & 1 & 2 & 1 & 1 & 2 & 0 & 1 & 1 \\
\hline jpical & & & & & & & & & \\
\hline & 3 & 1 & 4 & 3 & 1 & 4 & 5 & 1 & 6 \\
\hline & 0 & 0 & 0 & 0 & 0 & 0 & 0 & 0 & 0 \\
\hline & 1 & 2 & 3 & 0 & 1 & 1 & 1 & 0 & 1 \\
\hline & 1 & 0 & 1 & 0 & 0 & 0 & 0 & 0 & 0 \\
\hline & 2 & 0 & 2 & 1 & 1 & 2 & 1 & 0 & 1 \\
\hline & 0 & 0 & 0 & 0 & 0 & 0 & 0 & 1 & 1 \\
\hline & 1 & 2 & 3 & 1 & 2 & 3 & 0 & 2 & 2 \\
\hline & 0 & 1 & 1 & 0 & 1 & 1 & 1 & 1 & 2 \\
\hline & 0 & 2 & 2 & 1 & 2 & 3 & 0 & 2 & 2 \\
\hline & 0 & 5 & 5 & 0 & 5 & 5 & 1 & 4 & 5 \\
\hline 0 & & & & & & & & & \\
\hline & 0 & 0 & 0 & 0 & 0 & 0 & 1 & 0 & 1 \\
\hline & 6 & 3 & 9 & 6 & 2 & 8 & 6 & 2 & 8 \\
\hline
\end{tabular}

First, the individual patterns are arranged under Typical (left dominant), Atypical (bilateral or right dominant), or other category based on the spatial distribution of their activation centroids. The table also shows the LRE and control subject distribution. The "Other" category includes noise, null activation (also non-canonical activations). 
Table 4. 5: PCA Classification vs. Visual Rating Results of Three Raters

\begin{tabular}{|c|c|c|c|}
\hline Rater1 & Typical & Atypical & Total \\
\hline PCA-NDF & & & \\
\hline Typical & 78 & 3 & 81 \\
\hline Atypical & 14 & 27 & 41 \\
\hline Total & 92 & 30 & 122 \\
\hline
\end{tabular}

\begin{tabular}{|c|c|c|c|}
\hline Rater2 & Typical & Atypical & Total \\
\hline PCA-NDF & & & \\
\hline Typical & 76 & 5 & 81 \\
\hline Atypical & 19 & 22 & 41 \\
\hline Total & 95 & 27 & 122 \\
\hline
\end{tabular}

\begin{tabular}{|c|c|c|c|}
\hline $\begin{array}{c}\text { Rater3 } \\
\text { PCA-NDF }\end{array}$ & Typical & Atypical & Total \\
\hline Typical & 75 & 6 & 81 \\
\hline Atypical & 18 & 23 & 41 \\
\hline Total & 93 & 29 & 122 \\
\hline
\end{tabular}

A classification of typical and atypical activation comparing the PCA-NDF and the visual rating categorization by three raters respectively. The strength of the agreements ranged from $80 \%$ to $86 \%$, are considered significant (Kappa coefficient $(\kappa)=0.52$ to $0.67 ; p=0.05$ ). Note for comparison purpose with the nonlinear classifiers, the "Other" category in visual rating is considered "atypical".

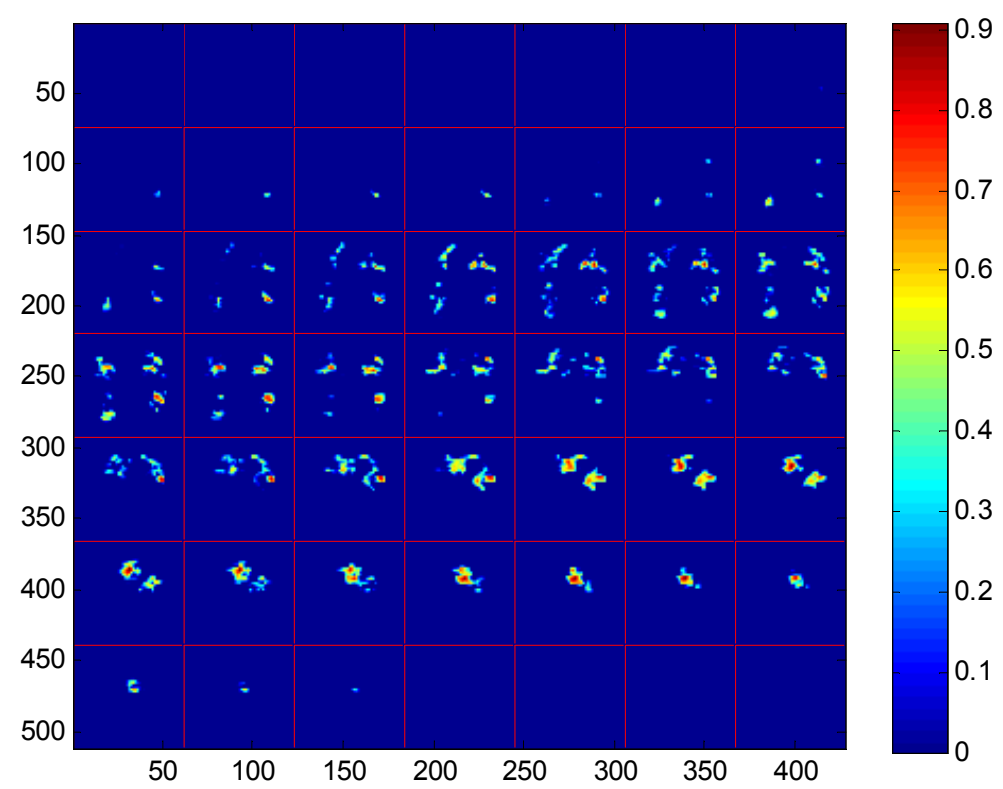

Figure 4. 10: Selected axial cuts for a mismatch case where visual rating categorized as pattern 1 (strong left lateralized both in Broca's and Wernicke's areas), which is classified as typical while the PCA-NDF classified it as atypical. 


\subsubsection{Clinical variables}

In our study, 79 subjects were found to be in agreement by all the methods (PCANDF, LI magnitude, LI extent, LI average, three raters). Among these agreed subjects, 33 controls (mean age 9.1 yrs, 18 male) and 33 patients (mean age 13.9 yrs, 18 male, 2 Ambidextrous handed, 30 right handed, seizure onset $8 \mathrm{yrs}$ ) are typical, while 3 control (mean age $7.64 \mathrm{yr}, 1$ male,) and 10 patients (mean age 14.2 yrs, 7 male, 6 left handed, seizure onset $9.1 \mathrm{yrs}$ ) are atypical. Left handed patients tend to have atypical activation patterns (Fisher exact test, $\mathrm{p}<0.001$ ).

\subsection{Discussion}

The main contribution of this chapter consists in proposing the integration of nonlinear pattern classifiers into a modified PCA feature space in order to optimize the classification of fMRI language related activation brain patterns. Since we are using real data sets for generating the synthetic data to conform in the best way possible to actual activations, the resulting bias is significantly reduced in contrast to using purely synthetic data sets. For training purposes, the language type label of each synthetic dataset was ascertained through random visual inspection which reduces the bias as well. This yields an effective way of assessing the performance of nonlinear classifiers (NDF, SVM) under different dimensional spaces, while assessing the merits of different kernels and different degrees of complexity. The proposed PCA-NDF method showed good agreement with both the subjective visual categorizations and the ROI-based LI distribution. Corollary contributions can be derived from the fact that such nonlinear 
classification methods are also known to be robust even when the data is acquired from different scanners and different sites. Consequently, the standardization of the fMRI data brain activation patterns and the algorithm used to seek uniformity in the various data format considered in this study were proven effective, making our adaptation of the PCA-NDF classifier method entirely data-driven.

Taking these caveats into account, we achieved an automatic grouping algorithm whose results agreed well with commonly used language asymmetry statistics. Heterogeneity increases in the mixed population since both control and patient subjects will each have a given proportion with an atypical activation pattern, introducing both normal variants and pathological variants. Through the PCA-NDF method, we found that typical group, shared by $66 \%$ of the entire population, presented high activation in the left canonical language areas. The atypical group was characterized by strong activation on Broca's area in the right hemisphere dominant response for language, mirroring the responses of the other cluster in the homologous left areas of the brain.

Some mismatches were observed between the PCA-NDF and the visual rating or LI. Compared with visual rating and LI categorization, the typical group included certain bilateral activation patterns and the atypical group included certain uni-bilateral but left dominant patterns. Though we did not have complete agreement between these methods, PCA-NDF properly identified the atypical right dominant group and was supported by visual rating and ROI-based LI. We did not have ultimate confirmation by either invasive means such as the IAT or ECS, or surgical outcome; however, invasive measures also have their limitations, and it may be difficult to establish which method is ultimately correct. Moreover, our main aim was to provide an automated method to 
perform classification of brain activation patterns as a support tool to assist the more subjective visual rating methods or LI-based methods, but with no intent to substitute them.

The distribution of language patterns observed in this dissertation is similar to prior studies on language dominance within normal volunteers employing either transcranial-Doppler or transcranial magnetic stimulation, and within LRE subjects using the intra-carotid amobarbital (Khedr et al.,2002; Knecht et al. ,2000; Kurthen et al. ,1994; Rasmussen and Milner ,1977; Risse et al. ,1997; Woods et al. ,1988; Wyllie et al. ,1991). Atypical language activation identified with fMRI is estimated to occur in 20$30 \%$ of patients with chronic epilepsy (Berl et al., 2005; Gaillard et al., 2007; Woermann et al., 2003; Yuan et al., 2006) comparable to the result of $21 \%$ found in our study according to clinical rating, $31 \%$ according to ROI-based LI, and $28 \%$ according to all the methods (rating, LI, PCA-NDF). Atypical language activation has also been found in the normal right handed population, $12 \%$ on clinical rating, $18 \%$ on LI, , and $8 \%$ according to all the methods (rating, LI, PCA-NDF), which is higher than previous findings (Pujol et al., 1999). This difference might be due to the young age of normal population compared to adult populations. Left handedness was found associated with atypical language activation pattern.

Disagreements occurred among the raters and the different methods of calculating the LI. The sensitivity and objectivity of the PCA-NDF for group separation would help to overcome human rating errors as well as the limitation of prior assumptions. Furthermore, instead of a blind clustering of presented population, the idea of using synthetic activation patterns helps the method to be language patterns -oriented. 
The data-driven mechanism using NDF was found to be effective at classifying typical and atypical language networks activation patterns, even from a heterogeneous population often acquired with different acquisition parameters. The integration of PCA with the NDF classification paradigm results in a data-driven method that is both accurate and computationally appealing (within few seconds in processing time after the weights of the decision function are generated in the training phase).

This proposed approach could promote objective assessments of large data sets and can serve to interrogate data for a multitude of clinical variables. Again, as a single site is unlikely to evaluate a sufficient number of patients to identify variant activation patterns, the consortium of imaging epilepsy satisfied the needed collaborative efforts. Thus a large sample, inter-subject variance can be more reliably characterized for control populations as well as heterogeneous patient groups. With the growing data source from the consortium, more meaningful synthetic data can be generated, thus a multi-class nonlinear decision function will be more practical as the next step, to break down the two classes into more specific categories, since 15 patterns, at least in theory, are needed to describe the lateralization of brain activation related to language tasks. 


\title{
CHAPTER V
}

\author{
Distance Method on PCA-Based Decisional Space
}

\subsection{Introduction}

Earlier findings have demonstrated that language activation is typically left dominant, but there are known variants (bilateral or right dominance) present in both right handed (5\%) and left handed (22\%) populations (Pujol et al., 1999; Rasmussen and Milner, 1977; Springer et al., 1999; Szaflarski et al., 2002; Woods et al., 1988). Moreover, patients with Localization Related Epilepsy (LRE - the medical term used to refer to subjects with a localized seizure focus) reveal greater occurrence of atypical language (20-30\%) based on quantitative ROI analysis (Frost et al., 1999; Gaillard et al., 2007; Woermann et al., 2003) at hemispheric or regional levels (Binder et al., 1996; Gaillard et al., 2002; Ramsey et al., 2001; Spreer et al., 2002) or simply through visual rating (Fernandez et al., 2001; Gaillard et al., 2002, 2004). Elaborating on such early findings motivated the need to design new methods to identify deviant language patterns not constrained by simple left-right language dominance as determined by ROI with a priori assumption or by visual rating through subjective means.

The focus of this chapter is thus placed on a new strategy of using a PCA-based decisional space and how the clustering characteristics play out in terms of both the chosen eigenvectors and the intensity of the activation patterns. It also reveals a unique decisional space that combines the LI with the clustering characteristics, all as a mean to segregate fMRI language patterns. The group-analysis method presented here may help to 
study brain changes that may reflect brain plasticity. Furthermore, the epilepsy patient population provides a means for validating these methods because of their known heterogeneity of language dominance (Berl et al., 2005; Price et al., 2006, 2005). Such an approach could augment the knowledge gained through different PCA-based approaches reported in the literature that have either attempted to maximize variability, integrated fMRI and DTI studies, (Caprihan et al., 2008; Suma and Murali, 2007), used 4D fMRI data sets (Andersen et al., 1999; Viviani et al., 2005), applied the k-means method (Mbwana et al., 2009) or neural networks (Samanwoy and Hojjat, 2008), and used the Scale Subprofile Model (SSM) normalization transformation (Alexander and Moeller, 1994; Moeller et al., 1987; Smith et al., 2006). In all of these attempts, the shared belief is that PCA remains a powerful data-driven method applicable to data sets with good scalability. The PCA can also overcome the need for prior assumptions and the subjectivity associated with visual rating methods that are known to be bias, tedious and prone to human error.

The specific aims of this chapter are:

- To develop a new PCA-based procedure that provides a decisional space from which sub-groups of distinct fMRI activation patterns are identified in a mixed population of control and patient subjects.

- To compare resulting sub-groups obtained based on the PCA algorithm to the results obtained through the LI method and by the visual rater's clinical categorization. As a measure of caution, we are neither trying to categorize each subject into simple leftright dominance to replace the conventional clinical methods, nor striving to separate normal subjects from patients, but to find deviant activation patterns and to identify 
distinct language patterns that might be associated with the normal variant and pathological variant conditions.

- To determine decisional spaces that are most suited for optimal clustering trends, and from which we will ultimately define the decision boundaries that delineate such clusters. These decisional spaces include (a) the use of only the most relevant eigenvectors as the axes of the decisional space, and (b) introducing the LI as one axis of this decisional space in combination with any of the relevant eigenvectors as the other $\operatorname{dimension}(\mathrm{s})$.

- To assess the merit of introducing the intensity of the activation patterns without normalization as an important element of the clustering trends.

\subsection{Method}

\subsubsection{PCA-based Decisional Space on Activation Maps}

As indicated earlier, PCA has been reported as the core analysis of SSM after the initial normalization transformation in the context of modeling regional patterns of brain function (Moeller et al., 1987; Smith et al., 2006; Alexander and Moeller, 1994). Based on the concept of subject loading, we performed the PCA on the given 122 fMRI activation maps with the particularity of forgoing altogether regional masking and intensity normalization. Then, we utilized the top two components (subject loadings) to separate three primary activation patterns groups and later, through the Euclidean

distance method, associate the remaining subjects to the primary groups that were already determined. After the top eigenvectors were generated following steps 1-3 of section 3.2 
of Chapter 3, detailed implementation steps of the modified Euclidean distance method are as follows:

1. Based on the $\mathrm{e}_{\mathrm{i}}$ distribution in the matrix $E$, three primary clusters with far distances from each other were first determined linearly. Then the new mean $\left(m_{\text {new }}\right)$ vector of these clusters was generated with subjects only chosen from the three primary clusters, and the PCs of these clusters were calculated, generating the new matrix $U_{\text {new }}$ following Eq.3.2.

2. Group the subjects' activation maps not falling in any of the primary clusters (undecided regions) using the distance method. Vector $x_{\text {new }}$ will now represent the activation map of the subject. Thus the distance method is used to determine which cluster is the closest to the undecided subjects. The following sub-steps are undertaken:

a). Project $\Phi_{\text {new }}$, which is the new centered $x_{\text {new }}\left(\Phi_{\text {new }}=x_{\text {new }}-m_{\text {new }}\right)$, onto the primary clusters defined eigenspace using Eq.5.1.

$$
\hat{\Phi}_{\text {new }}=\sum_{l=1}^{j} u_{l}^{T} \Phi_{\text {new }} u_{l}
$$

where each $u_{l}$ representing a column vector of the $U_{\text {new }}$ matrix.

b). Calculate the Euclidean distance feature using Eq.5.2 below:

$$
D_{i}=\left\|\hat{\Phi}_{\text {new }}-\Phi_{i}\right\|
$$

for $i=1,2, \ldots ., q$, where $q$ is the number of primary cluster members, with $\Phi_{i}=x_{i}-m_{\text {new }}$ and where $j(j<k)$ is the number of eigenvectors selected. In this approach, $j$ was tried from 3 to 7 , and the separation results were found to be the same, which clearly 
demonstrates that the top eigenvectors already include enough information of the population.

c).The new subject $\Phi_{i}$ was assigned to the cluster whose member had the minimum distance as calculated in Eq.5.2. In other words, the new subject is assigned to the cluster where the closest identified subject $\Phi_{i}$ was located.

\subsubsection{Analysis of Segregation Results}

Fisher exact test was applied to assess the site independence as well as the significance of association for signal intensity grouping vs. either magnet strength or control/patient grouping.

In order to corroborate the PCA clustering results, each subject's activation map was visual rated by three investigators blinded to subject identity, in order to score the laterality of activation in the language network related areas. The rating tool and procedures were described in chapter 2. Moreover, LI was calculated using Eq.2.1 provided earlier with $V$ being the activation magnitude.

\section{$5.3 \quad$ Results}

\subsubsection{Blind source data clustering}

After obtaining the PCA eigensystem, the dendrogram of the $E$ matrix suggests indeed that there are three major groups within these 122 subjects as shown in Figure 5.1 (Stanberry et al., 2003; Mbwana et al., 2009). Along with the observations made on the merits of the second eigenvector in section 3.3.3, the first eigenvector is also found to 
separate higher intensity from lower intensity when considering only the typical subjects that satisfied the condition $e_{2}>0$ as seen in Figure 5.2. This plot reflects the subjects' distribution based on intensity and the -0.1 value for $e_{1}$ was chosen as the primary cluster threshold for the higher intensity group and 0 for lower intensity group. Therefore, $e_{1 i}<-$ $0.1 \cap e_{2 i}>0$ were chosen as the boundary for primary cluster 2 (the higher intensity typical group), while $e_{1 i}>0 \cap e_{2 i}>0$ were chosen as the boundary for primary cluster 1 (the lower intensity typical group).

Later, the subjects located in the indeterminate region ( $\mathrm{n}=75)$ (subjects not belonging to any of the three primary clusters) were projected onto the new eigenspace that was generated by the PCA on the three primary clusters following Eqs. 5.1 and 5.2. Then each of these 75 subjects was assigned to their closest cluster using the distance method. Figures 5.3 and 5.4 depict the clustering results achieved by utilizing the top 2 eigenvectors as criteria to select primary clusters, and the distance to the top 3 eigenfaces to assign undecided subjects. It is worthy to note that the separation results obtained by using 3 up to 7 eigenfaces were found to be identical.

\subsubsection{Identified Activation Patterns}

The final clusters' mean activation patterns are shown in Figure 5.5. The strongly activated areas found in these three types of activation patterns (in relation to the three clusters) broadly encompass Broca's and Wernicke's areas. As anticipated, group 1 (Figure 5.5a) was the typical language response on the left hemisphere while group 3 (Figure 5.5c) had an atypical strong right hemisphere dominant response. Most of the 
subjects from groups 2 and 3 were patients, 15 patients out of 18 for group 2, and 8 out 9 for group 3, while control subjects are dominant in group 1 (60/95) (Figure 5.4). Group 2 (Figure 5.5b) consisted of a group of cases that shared the same areas as group 1. However, the procedure was able to distinguish group 2 from group 1 because group 2's activation intensities were much higher than those of group 1, especially in Broca's area. Note that groups 2 and 3 are variants compared to major group 1, and group 3 is closer to current notion of atypical language activation pattern which is atypical bilateral or right dominant.

To examine the correlation between LRE patients and group 2, we presented the LRE-control distribution inside group 1 and group 2 in Table 5.1. LRE patients (group 2) were found to show greater activation than subjects in group 1 (although the former present with typical language localization as well) (Fisher exact test: $\mathrm{p}=0.0004)$.

To ascertain whether magnetic strength plays a role in group segregation, Table 5.2 was created to contrast groups 1 and 2 with group 3 on the basis of magnetic strength (group 1 and 2 are considered both typical language in PCA). We found no difference in the effect of scanner magnetic strength in group segregation of laterality category (Fisher exact test, $\mathrm{p}=0.7$ ). Since no control subjects scanned by $1.5 \mathrm{~T}$ and group 1-group 2 correlated with control-LRE, it is natural that 3T-1.5T correlated with group 1group 2. Hence there is a magnet strength vs. group 1-2 correlation considering both control and patients (Fisher exact test, $\mathrm{p}=0.0005$ ). This correlation found here is a natural consequence due to the imbalance of subject population. It was confirmed that the magnet difference did not relate to the group within LRE subjects when only patients are taken into account (Fisher exact test, $\mathrm{p}=0.2$ ). 
To ascertain whether group segregation is independent of data collection sites, Table 5.3 was created to contrast groups 1 and 2 with group 3 on the basis of sites. The concatenation of group 1 and 2 is because control subjects were scanned at only one site and group 1-2 difference was found correlated with control-LRE difference. We found no difference between the effect of sites in group segregation (A Good Fit Test, $\mathrm{p}=0.58)$.

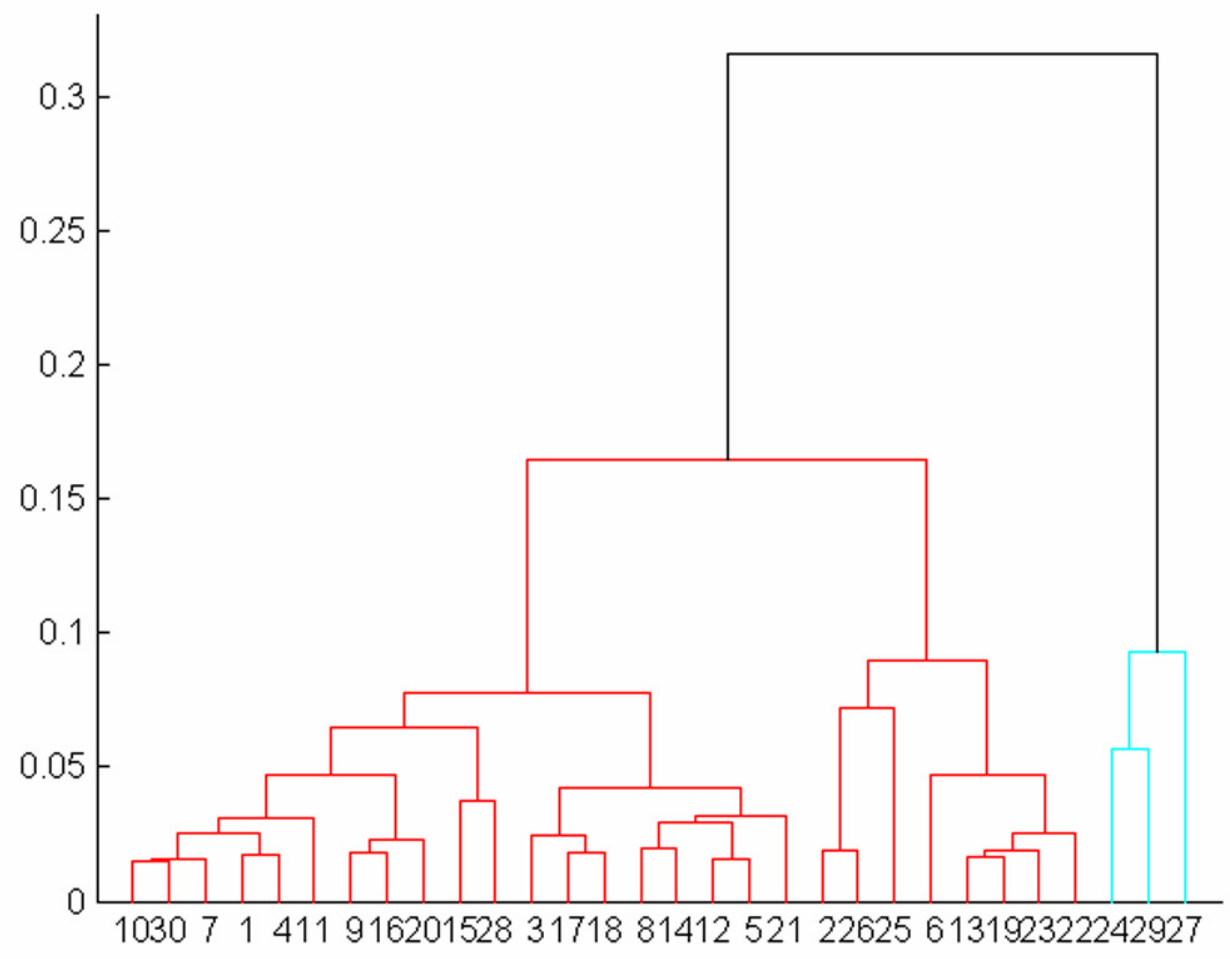

Figure 5. 1: The dendrogram of the Euclidian distance matrix of the PCA suggesting there are at least three subgroups within the subjects. 


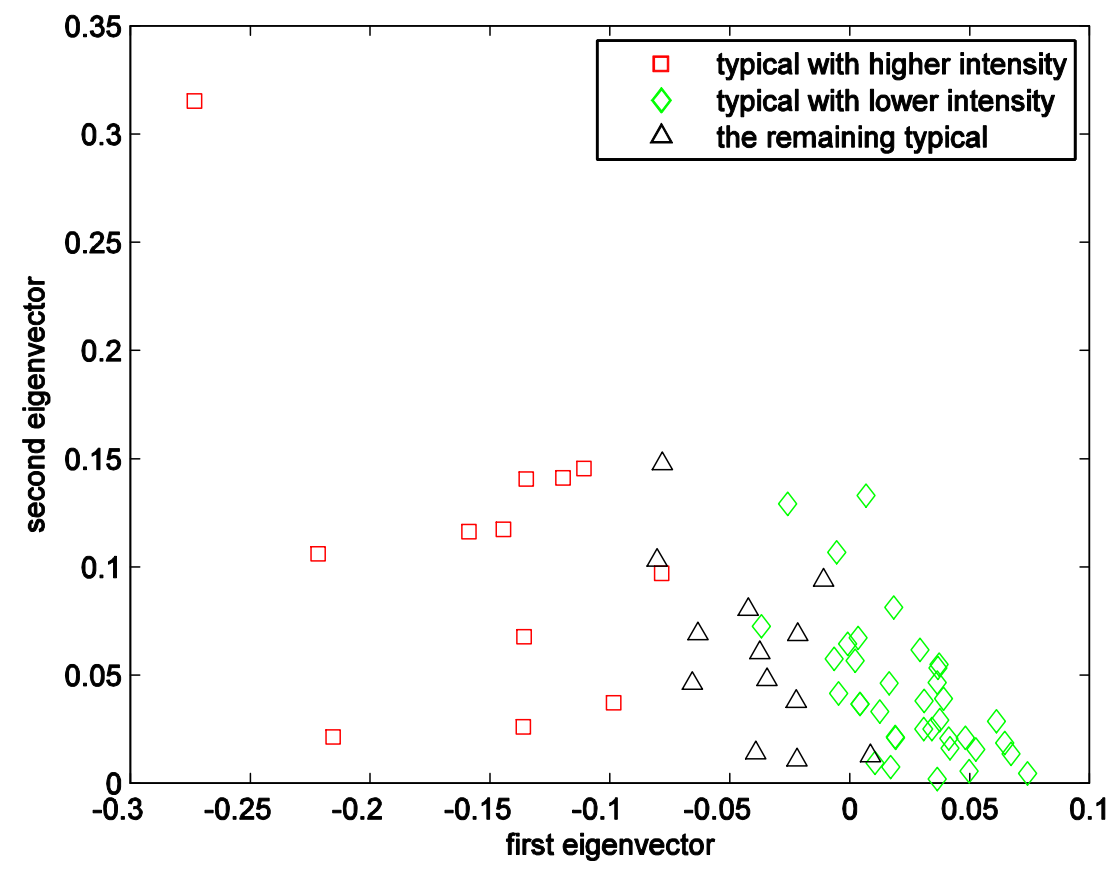

Figure 5. 2: Considering only the typical subjects that satisfied the condition $\mathrm{e}_{2}>0$, this plot reflects the subjects' distribution based on intensity. The red squares are those subjects whose intensities are higher than the mid-point of the intensity range of the analyzed population's means; green diamonds are the ones that are lower than the mean activation intensity of these typical subjects.

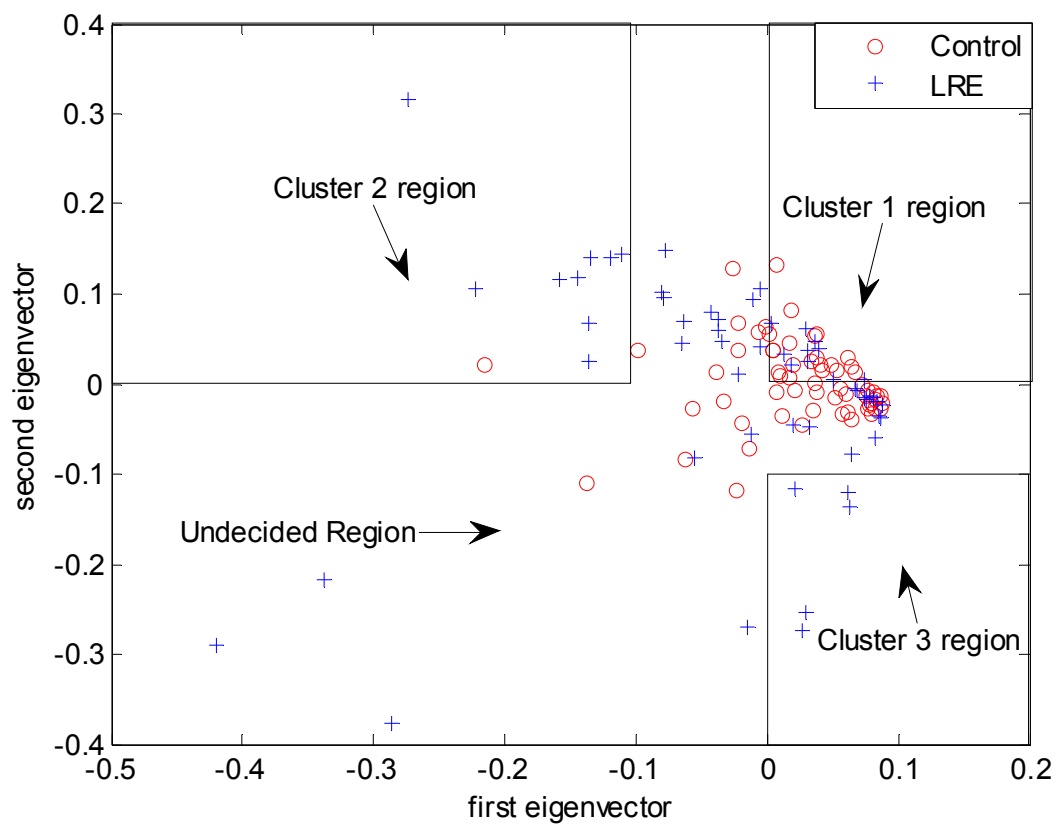

Figure 5. 3: Primary clusters using the two dominant eigenvectors of the PCA. These two dominant eigenvectors are used to select three primary clusters based on the following decision rules: cluster 1: the most condensed cluster region with 32 data points; cluster 2: with ten data points; cluster 3: with five data points. The undecided region, with 75 data points, is the remaining region outside these three clusters. 


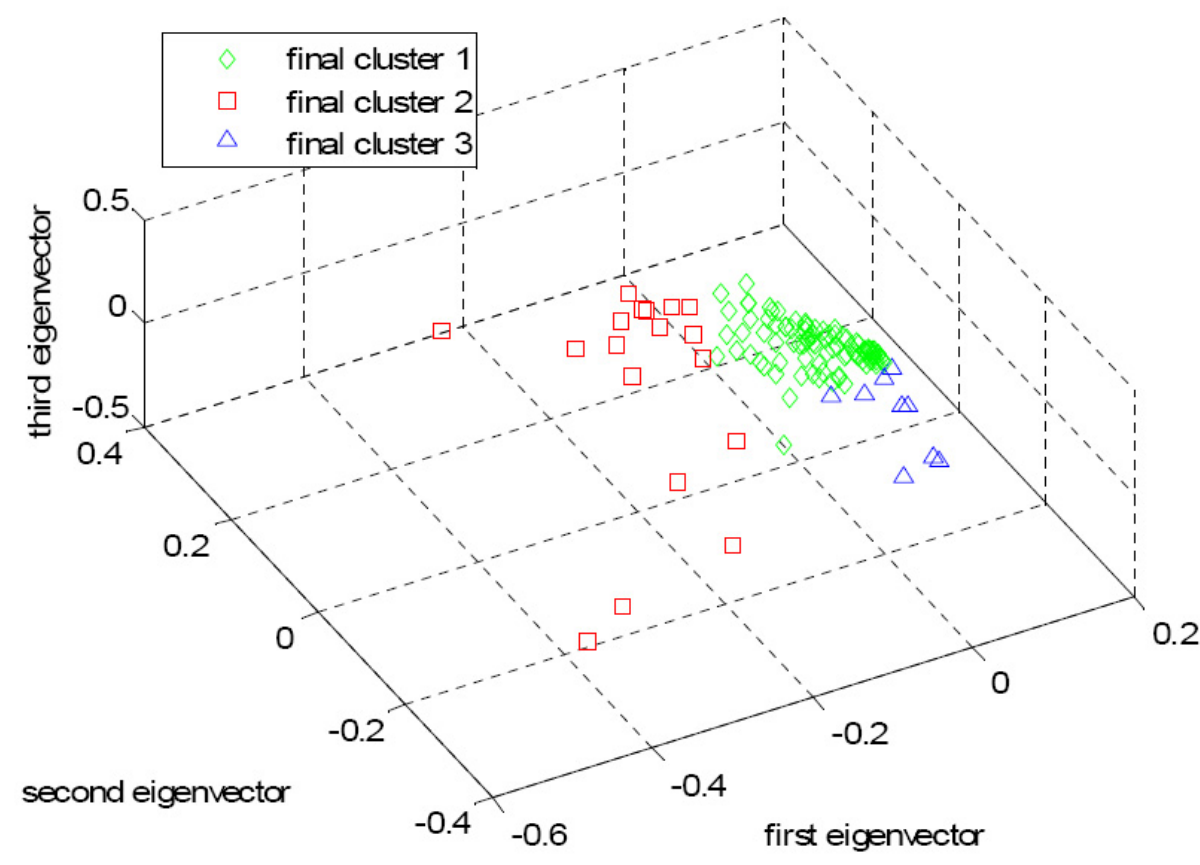

Figure 5. 4: Clusters distribution achieved using two eigenvectors. Note, data points corresponding to former undecided subjects are now assigned to one of the primary clusters based on the distance method.

(a)

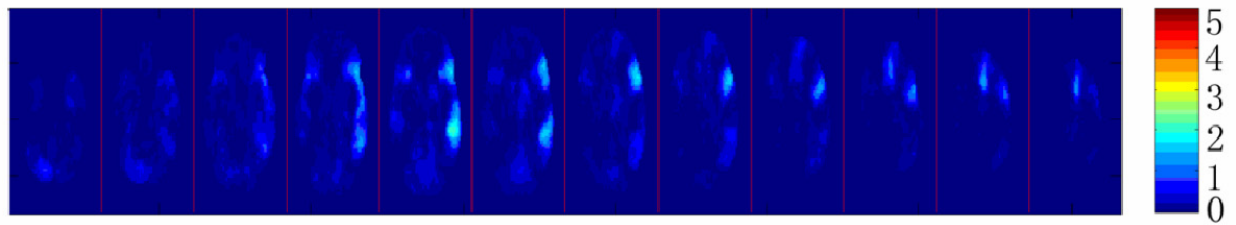

(b)

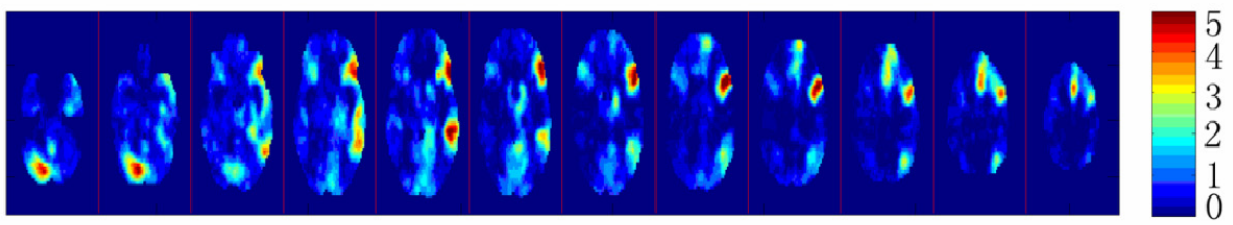

(c)

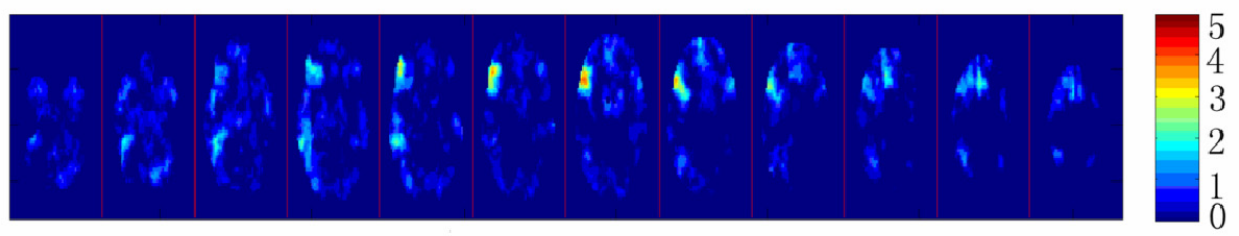

$\begin{array}{llllllllllll}12 & 15 & 18 & 21 & 24 & 27 & 30 & 33 & 36 & 39 & 42 & 45\end{array}$

Figure 5. 5: Mean activation maps for each final cluster/group. 2D array of selected axial cuts color coded for activation intensities. Higher activations are in red color. Brain oriented in radiological convention: left hemisphere on the right side. The number below the image is the slice number. (a) Mean activation map for group 1. (b) Mean activation map for group 2.The $\mathrm{z}$ value range is higher than (a). This explains the better definition of Supplementary Motor Area (SMA). (c) Mean activation map for group 3 with an atypical right hemisphere dominant response. 
Table 5. 1: Distribution of LRE vs. control subjects in typical groups

\begin{tabular}{cccc}
\hline & \multicolumn{3}{c}{ Number of Subjects } \\
\cline { 2 - 4 } Group & LRE & Control & Total \\
\hline 1 & 35 & 60 & 95 \\
2 & 15 & 3 & 18 \\
Total & 50 & 63 & 113
\end{tabular}

Group 1 and 2 are left lateralized, but group 2 consists of higher intensities. As a result, SMA is more evident. A value of 0.0004 was found when applying Fisher exact test, indicating strong correlation between LRE subjects and group 2.

Table 5. 2 : Magnet scanner vs. group category

\begin{tabular}{c|ccccccc}
\hline & \multicolumn{2}{c}{$3 \mathrm{~T}$} & \multicolumn{2}{c}{ 1.5T } & \multicolumn{2}{c}{ Total } \\
Group & LRE & Control & LRE & Control & LRE & Control \\
\hline 1 & 17 & 60 & 18 & 0 & 35 & 60 \\
2 & 4 & 3 & 11 & 0 & 15 & 3 \\
3 & 4 & 1 & 4 & 0 & 8 & 1 \\
Total & 21 & 64 & 33 & 0 & 58 & 64
\end{tabular}

$\mathrm{T}=$ Tesla. No effect of magnetic strength on laterality clustering results was demonstrated (Magnetic strength vs. laterality category (group $1+2$ to group 3 ) Fisher exact test: $p=0.7$ ). No significant effect of magnetic strength on separating patients in group 1 and group 2 (intensity) (Fisher exact test: $p=0.2$ )

Table 5. 3: Sites vs. Group Category

\begin{tabular}{c|ccccc}
\hline Sites & & & & & \\
Group & A & B & C & D & Total \\
\hline Group 1+2 & 9 & 12 & 10 & 17 & 48 \\
Group 3 & 2 & 2 & 0 & 2 & 6 \\
Total & 11 & 14 & 10 & 19 &
\end{tabular}

Compared sites with 10 or more patients. No effect of sites on clustering results was demonstrated (A Good Fit Test, $\mathrm{p}=0.58$ ). 


\subsubsection{LI vs. PCA Clustering Results}

In order to show the relationship between the LI and the PCA result, a 2D plane was used to describe the distribution of clustering results considering individually the LI obtained based on the activation of the Broca's and Wernicke's areas. The $x$ axis indicates the LI obtained on the Broca's area while the $y$ axis indicates the LI obtained from activations on the Wernicke's area.

Figure 5.6 plots the distribution of cluster s associated with the LI of Broca's and Wernicke's areas. This figure shows that clusters 1 and 2 are associated with positive LI values (left lateralization) and that cluster 3 is associated with high negative values. The data also demonstrate that from the nine subjects in cluster 3, eight LRE subjects are associated with strong right-brain dominant activation while only one healthy control presents atypical bilateral activation. In conclusion, the results reveal that the LI value calculated using the bootstrap method will not strictly agree with the cluster results when using the PCA, but LI will concur with the major trend of PCA in finding the most atypical activation pattern (which is right dominant in our case).

Table 5.4 presents the classification of the 122 subjects according to both methods: PCA and LI. This categorization assumes a classification of non-right and atypical right activation. In order to compare PCA and LI in a reasonable fashion, since PCA is looking at deviant patterns while LI is looking at dominance and will not be able to tell the intensity differences, so we concatenated group 1 and group 2 of PCA which are both non-right dominant in LI's point of view, and we adapted LI's classification category into right dominant vs. non-right dominant also. The strength of the agreement, $96.7 \%$, is considered "substantial" (Kappa $=0.76 ; \mathrm{p}=0.05 ;$ CI: [0.5288, 0.9913]). 


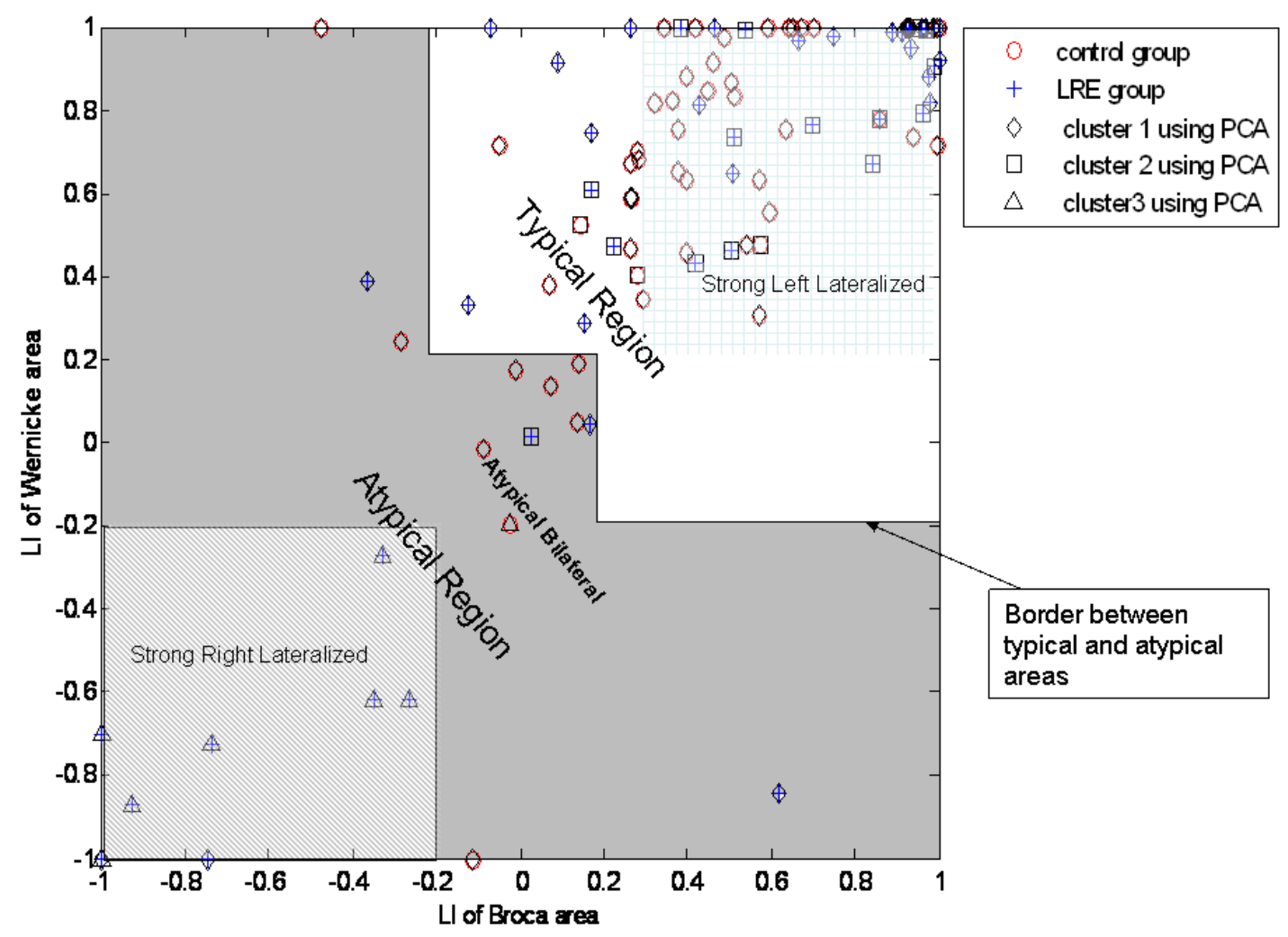

Figure 5. 6: Distribution of PCA clustering results in LI of frontal and temporal language areas. "Strong right lateralized" is determined when both the LI of Broca's and Wernicke's areas are lower than -0.2. "Strong left lateralized" is determined when both the LI of Broca's and Wernicke's areas are higher than 0.2 . The gray area is an atypical region including strong right lateralized and atypical bilateral. 7 out of 9 members of cluster 3 are inside the strong right dominant region.

Table 5. 4: PCA Segregation vs. LI Categorization Results

\begin{tabular}{cccc} 
LI PCA & $\begin{array}{c}\text { Non-Right } \\
(\mathrm{G} 1+\mathrm{G} 2)\end{array}$ & $\begin{array}{c}\text { Right } \\
\mathrm{G} 3\end{array}$ & Total \\
\hline Non-Right & 111 & 2 & 113 \\
Right & 2 & 7 & 9 \\
Total & 113 & 9 & 122
\end{tabular}

G1, G2, and G3 = Group 1, 2 and 3 respectively. Group 1 and group 2 in the PCA results were categorized as non-right dominant language responses, while group 3 was right dominant. Lateralization agreement was found in 118 of 122 subjects (Kappa $=0.76 ; \mathrm{p}=0.05$;CI: [0.5288,0.9913]). 


\subsubsection{Visual rating Results vs. PCA cluster Results}

The inter-rater agreement among the three raters was found almost perfect (Fleiss Kappa: 0.8132, $\mathrm{p}=0.05$ ) with their distribution shown in Table 5.5. Their agreement with PCA results under the right vs. non-right dominant categorization is provided in Table 5.6. Note that the non-canonical activation was depicted here as no activation. The agreements were found significant when comparing each rater's result to PCA's (Kappa ranged from 0.54 to $0.70, \mathrm{p}=0.05$ ).

There is a partial disparity among visual rating itself, which suggests its subjectivity from Table 5.5. Partial disparities were also found from the comparison between visual rating and the PCA, and also between the PCA and the LI, as seen in Table 5.6 and Table 5.4. The explanation for this disagreement is that, in contrast to visual rating which is a clearly anticipated pattern-oriented process, PCA applied here only tries to separate the three groups based on their activation pattern differences. Therefore, this method does not guarantee that the three groups will be precisely left dominant, right dominant or bilateral. Same comparison was done between LI and visual rating, the agreements were similar to the one previously found between PCA and visual rating.

\subsubsection{Other methods vs. Euclidean}

It is worthy to look into these other methods, as a way to support that the three clusters that we found through our modified Euclidean Distance method indeed provide a good separation as further evidence of the dendrogram given in Figure 5.1. In this section we describe the implementation of the discriminant analysis. In this 
implementation, we found that the linear discriminant function performed the best in delineating our final clustering results (using the MATLAB statistic toolbox). Figure 5.7 depicts the linear discriminant function that fit our PCA separation results.

Then we tried the discriminant analysis method for the modified Euclidean distance step to classify the undecided subjects into the three primary clusters. Just to compare the two methods, after defining the primary clusters using the PCA system, we used the primary clusters as training data sets, and the rest (75) data sets as samples to feed in the classifiers. Table 5.7 shows the results obtained using discriminant analysis on the remaining 75 data sets. As seen in this table, the modified Euclidean distance method is stable from 3 through 7 input eigenvectors, while discriminant analysis slightly changes with the linear discriminant function chosen. The classification results of both methods are however comparable. We can observe that cluster 2 members remained the same. We identified six mismatch cases: two assigned to cluster 1 (expected to be left dominant) and four assigned to cluster 3 (expected to be right dominant) in the final classification of the undecided subjects. The former 2 cases were initially categorized as right dominant (cluster 3) which was supported with both visual rating and LI, three out of four cases were initially categorized as left dominant which was also supported by visual rating/LI. The remaining mismatch case was previously categorized as left dominant by our method and declared bilateral activation by visual rating/LI. Therefore, it shows that our method performs closer to the actual characteristics of the activation patterns. Figures 5.7 and 5.8 allow us to compare the final classification results using both methods. Similar procedures were tried through SVM instead of discriminant analysis; we found that the classification results of SVM are not better correlated to clinical measures compared to our modified Euclidean method. 


\subsection{Discussion}

Different PCA-based methods have been utilized to identify fMRI activation patterns (Andersen et al., 1999; Viviani et al., 2005) but only at an intra-subject level. fMRI activation analysis at the inter-subject level has been utilized by Werder et al. (2006) in a study of a few subjects in which they aimed at separating epilepsy patients from control subjects (Weder et al., 2006). Seghier et al. (2007 and 2008) also used an inter-subject approach by applying a fuzzy clustering algorithm to detect subject-specific activations to an fMRI lexical reading test in 38 normal subjects; using different variance analysis, they found sub-patterns of activations that were related to different skill sets or cognitive strategies. Mbwana et al. (2009) identified four patterns of activation among 45 patients with left hemisphere seizure foci based on PCA clustering following difference maps to see how individuals deviated on a voxel-wise basis from a normal control group. They found evidence for intra-hemispheric compensation and interhemispheric reorganization in three patient subgroups. However, their results were obtained after necessarily excluding the controls with atypical activation; only heterogeneity of the patient population was considered. Ford et al. (2003) also attempted to classify patients' fMRI activation maps but with a different method and in different areas, using the Fisher Linear Discriminant for Alzheimer's disease, schizophrenia, and mild traumatic brain injury(Ford et al., 2003). Suma et al. (2007) have also demonstrated that PCA can be used for the classification of fMRI activation maps; however, PCA was not directly applied to activation maps, but rather to area and centroid values obtained from post-processing the activation maps on a slice basis. 
In this chapter we use a PCA-based decisional space to recognize sub-groups of distinct language activation patterns in control and LRE patients from different sites. All subjects performed the same ADDT fMRI task. Based on the results obtained, three subgroups were identified: 1) the typical distributed network expected for task in left inferior frontal gyrus (Broca's) and left superior temporal gyrus (Wernicke's) (60 controls, 35 patients); 2) a variant left dominant pattern with greater activation in IFG, mesial left frontal lobe, right cerebellum (3 controls, 15 patients); and 3) Activation in the right homologues of the first pattern in Broca's and Wernicke's territories (1 control, 8 patients). Normal controls were predominantly in group 1; Patients fell into the three groups but distribution was different than controls, and were over represented in groups 2 and $3(\mathrm{P}<0.0004)$. The two left dominant groups differed on the intensity level of regional activations. The typically developing control children primarily were in group 1 while mostly patients belonged to group 2 . These findings may represent an effect of epilepsy, its underlying substrate on language network expression or may represent different strategies in performing the task (Berl, et al. 2005). The proposed method showed good agreement with both the subjective visual categorization and the ROI-based LI distribution in identifying the strong right dominant group, when sub-groups, which shared localization but had intensity differences, were joined as one group.

The merit of PCA eigenvectors has been explored in few fMRI studies, both in a confirmatory and a classifier manner, which are different from our study. Sugiura et al. successfully used the loadings of PCA for separating fMRI activation regions into three groups from 19 normal subjects on memory-guided saccade tasks (Sugiura et al., 2004). Their analysis was based on the assumption of the homogeneity of the normal population 
and required a priori knowledge of predefined ROIs as well as each region's relationship to the three main lobes. In another study, PCA with reference (PCA-R) combined with coefficient-constrained independent component analysis (CC- ICA) were used as classifiers to distinguish 28 schizophrenia patients from 25 healthy controls based on results of sensorimotor tasks (Sui et al., 2009). Thus, their study presumed foundamental differences between patient and control populations.

Though the PCA we used is a standard feature extraction approach, our implementation differs from other methods in several ways. For each subject in our method, the entire activation map was fed into the algorithm, without intensity normalization. Potential differences in language patterns based on extent and intensity may thus be identified. Furthermore, data segmentation was performed without a priori assumptions or subject classification: we combined typically developing and patient populations to allow the algorithm to associate statistical features based on the data and therefore overcoming subjectivity imposed by using selected normal subject as reference. Within the mechanisms of this data driven method, mathematical thresholds were uniquely derived to delineate regions for three primary clusters based on the first two eigenvectors of the PCA; while the modified-Euclidean distance method is used to classify the undecided subjects into one of the three primary clusters initially determined, by projecting them into the new eigenspace of these primary clusters.

The motivation here is to determine to which primary cluster the activation patterns of the undecided subjects most resemble. The advantage is that the final clustering results are not grouped randomly, but taking into consideration both the most significant feature difference (top eigenvectors for primary clusters) as well as the voxel- 
to-voxel statistical difference in 3D images. With the increasing number of fMRI data sets made available through the consortium, the PCA-based data driven method is well positioned to reliably identify sub-patterns of language processing from the pooled data.

The differences in scanner manufacturer, magnetic strength and acquisition parameters are often perceived as limitations that hinder group analysis on the data sets collected from a variety of sites. Standard post-processing group analysis discourages the utilization of different scanners, different settings, and different resolutions; however, the methods used for this study provide standardization for different formats and our analysis showed that there was no scanner or site effects in our clustering results. These findings support collaborative efforts to investigate patient populations that require substantial number of subjects to gain more insights from the heterogeneity.

Taking these caveats into account, we achieved a grouping algorithm whose results agreed well with commonly used language asymmetry statistics in finding the right dominant group and identifying a group with higher intensity that the general LI and visual rating methods neglect. Logically, the chances of getting heterogeneity will increase with the size of the mixed population used since both control and patient subjects will have a given proportion with an atypical activation pattern, introducing both normal variants and pathological variants. Group 3 was characterized by a right hemisphere dominant response for language, mirroring the responses of the other two groups in the homologous left areas of the brain, which is the most extreme version of atypical language representation.

The distribution of language patterns obtained is consistent with prior studies on language dominance in normal volunteers using either transcranial-Doppler, or 
transcranial magnetic stimulation, or in LRE patients using the IAT (Khedr et al., 2002; Knecht et al., 2000; Kurthen et al., 1994; Rasmussen and Milner, 1977; Risse et al., 1997; Woods et al., 1988; Wyllie et al., 1991).

The intensity-based separation given by the PCA method of the same localized groups (1 and 2) may be considered another advantage for the implementation of this proposed method (Knecht et al., 2000). Group 2 remains left hemisphere dominant, but the areas distribution and intensity of activation are different than Group 1. There is greater activation in cerebellum inferior in mid frontal gyrus which means that group 2 is a variant pattern for task. Indeed, our method will discriminate two identical activation maps based on intensity differences.

The chosen $\mathrm{p}$ value used in generating the activation maps in the data preprocessing section might also affect the grouping result, especially the visual rating. Therefore, the ultimate validity of the interpretation of the clustering results depends on the algorithm that will generate the individual activation map representing the language network of each subject, before performing a group analysis. A p value of 0.05 cluster corrected with $\mathrm{Z}=2.3$ thresholding is normally acceptable. However, to obtain viewable activation results, which matters for the visual rater (Binder et al., 1996; Gaillard et al., 2004, 2002), some data sets require rating at less rigorous thresholds than $p<0.05$ corrected.

Some mismatches were observed between the PCA and the visual rating or LI as shown in Table 5.6 and Figure 5.6. Some of these subjects were rated with a low certainty level ( 5 or 4$)$. The other case may be explained by differences in thresholding: PCA uses only raw intensity values while the visual rating is based on post-processed 
thresholded images. For example, a weak activator's thresholded image may be dominated by the peaks (maxima) while the raw data utilized by the PCA method account for all the activated voxels (extent). This difference could explain cases in which higher values are localized to the right (as rated visually), but sub-thresholded raw values are more numerous in the left as PCA indicates. Furthermore, PCA is sensitive to intensity difference and does not cluster based on lateralization, rather the eigenvectors which we have presumed relate primarily to this feature; hence, some cases may segregate differently. It is noted there are also disagreements among the three raters, as well as their categorizations with LI. Though we did not have complete agreement between these methods, PCA properly identified the right dominant group and was supported by visual rating and ROI-based LI.

There are some limitations to our study. The segregation process for the intermediary value may be imperfect, since the boundaries of the primary clusters were defined based on the relationship between the top eigenvectors and the hemispheric dominance as well as between the top eigenvectors and intensity. The decision in terms of number and threshold criteria for primary cluster is based on the characteristics of our analyzed population. Thus, the boundary calculated to identify primary clusters is valid only for a mixed population with high variability of activation intensity and broad distinction of left and right hemisphere dominance. This limitation was somewhat attenuated given that the dendrogram identified three major groups present in our mixed population. It is also possible that some, less common, variant sub-patterns were not identified. Based on a supervised process, we identified $39 \%$ of the population into primary clusters. These primary clusters were used as references for a second round 
classification to sort the undecided data sets and associate them to the closest cluster. These undecided subjects did include variant activation patterns, such as bilateral activation, not represented in a straight forward manner in the primary clusters but scattered in the decisional space.

The PCA-based method presented here becomes a cluster tool that may help the assessment of very large data sets where visual or ROI rating may be unpractical or difficult. The method would be cost-effective and could be used as a means to interrogate data for clinical variables. Future research may also take advantage of the PCAdecisional space characteristics for group separation in order to overcome human rating errors or rigid paradigms of interpretation that perhaps too narrowly limit brain language activation into simple patterns of left, bilateral or right lateralization. As a note of caution, PCA space depends on the sampled population. If the PCA were applied only in the control group or the LRE group, different decisional space would be found. Therefore, the current results are valid only for the mixed population.

Furthermore, future studies should evaluate either the convenience of merging similar groups based on clinical need of simple lateralization categorization, for example groups of similar spatial distribution but segregated on grounds of intensity, or the merit of increasing even further the number of groups for research purposes. It might be worthy to break down the three clusters into more specific clusters within the PCA-decisional space, since, at least in theory, 20 patterns are needed to describe the lateralization of brain activation related to language tasks, although, it is neither practical nor necessary to consider all 20 clusters. Therefore, the next challenges are 1) to automate the process to determine a reasonable number of clusters and to decide the correct threshold for 
segregation criteria of each eigenvector in order to define other patterns of atypical language activation such as those localized in the neighboring non-canonical areas, and 2) to investigate the effects in extent and peak difference within the same hemisphere.

Table 5. 5: The pattern of distribution as identified by the three raters.

\begin{tabular}{|c|c|c|c|c|c|c|c|c|c|}
\hline \multirow[b]{2}{*}{ Non-R. } & \multicolumn{3}{|c|}{ Rater 1} & \multicolumn{3}{|c|}{ Rater 2} & \multicolumn{3}{|c|}{ Rater 3} \\
\hline & Control & LRE & Total & Control & LRE & Total & Control & LRE & Total \\
\hline & 37 & 30 & 67 & 38 & 31 & 69 & 39 & 33 & 72 \\
\hline & 1 & 3 & 4 & 2 & 4 & 6 & 1 & 4 & 5 \\
\hline & 5 & 3 & 8 & 7 & 2 & 9 & 4 & 3 & 7 \\
\hline & 6 & 5 & 11 & 4 & 5 & 9 & 4 & 4 & 8 \\
\hline & 1 & 1 & 2 & 1 & 1 & 2 & 0 & 1 & 1 \\
\hline & 3 & 1 & 4 & 3 & 1 & 4 & 5 & 1 & 6 \\
\hline & 0 & 0 & 0 & 0 & 0 & 0 & 0 & 0 & 0 \\
\hline & 1 & 2 & 3 & 0 & 1 & 1 & 1 & 0 & 1 \\
\hline & 1 & 0 & 1 & 0 & 0 & 0 & 0 & 0 & 0 \\
\hline & 2 & 0 & 2 & 1 & 1 & 2 & 1 & 0 & 1 \\
\hline & 0 & 0 & 0 & 0 & 0 & 0 & 0 & 1 & 1 \\
\hline & 1 & 2 & 3 & 1 & 2 & 3 & 0 & 2 & 2 \\
\hline Stro & Control & LRE & Total & Control & LRE & Total & Control & LRE & Total \\
\hline & 0 & 1 & 1 & 0 & 1 & 1 & 1 & 1 & 2 \\
\hline & 0 & 2 & 2 & 1 & 2 & 3 & 0 & 2 & 2 \\
\hline & 0 & 5 & 5 & 0 & 5 & 5 & 1 & 4 & 5 \\
\hline Other & & & & & & & & & \\
\hline & 0 & 0 & 0 & 0 & 0 & 0 & 1 & 0 & 1 \\
\hline & 6 & 3 & 9 & 6 & 2 & 8 & 6 & 2 & 8 \\
\hline
\end{tabular}

First, the individual patterns are arranged under Non-Right (Non-R.), Strong Right (Strong R.), or other category based on the spatial distribution of their activation centroids. The table also shows the LRE and control subject distribution. The "Other" category includes noise, null activation, and non -canonical activations. The inter-rater agreement among the three raters (Fleiss Kappa: 0.8132, p=0.05) 
Table 5. 6: PCA Separation vs. Visual Rating results of Three Raters

\begin{tabular}{cccc}
\hline Rater 1 & $\begin{array}{c}\text { Non-Right } \\
(\mathrm{G} 1+\mathrm{G} 2)\end{array}$ & $\begin{array}{c}\text { Right } \\
\mathrm{G} 3\end{array}$ & Total \\
\hline Non-Right & 113 & 4 & 117 \\
Right & 0 & 5 & 5 \\
Total & 113 & 9 & 122
\end{tabular}

\begin{tabular}{cccc}
\hline Rater 2 & $\begin{array}{c}\text { Non-Right } \\
(\mathrm{G} 1+\mathrm{G} 2)\end{array}$ & $\begin{array}{c}\text { Right } \\
\mathrm{G} 3\end{array}$ & Total \\
\hline Non-Right & 113 & 4 & 117 \\
Right & 0 & 5 & 5 \\
Total & 113 & 9 & 122
\end{tabular}

\begin{tabular}{cccc}
\hline PCA & $\begin{array}{c}\text { Non-Right } \\
(\mathrm{G} 1+\mathrm{G} 2)\end{array}$ & $\begin{array}{c}\text { Right } \\
\mathrm{G} 3\end{array}$ & Total \\
\hline Non-Right & 111 & 2 & 113 \\
Right & 2 & 7 & 9 \\
Total & 113 & 9 & 122
\end{tabular}

A classification of Non-right and right activation comparing the PCA and the visual rating categorization. G1, G2, and G3 = Group 1, 2 and 3 respectively. The strength of the agreements ranged from $95.08 \%$ to $96.72 \%$, are considered substantial (Kappa $=0.54$ to $0.70 ; \mathrm{p}=0.05$ ).

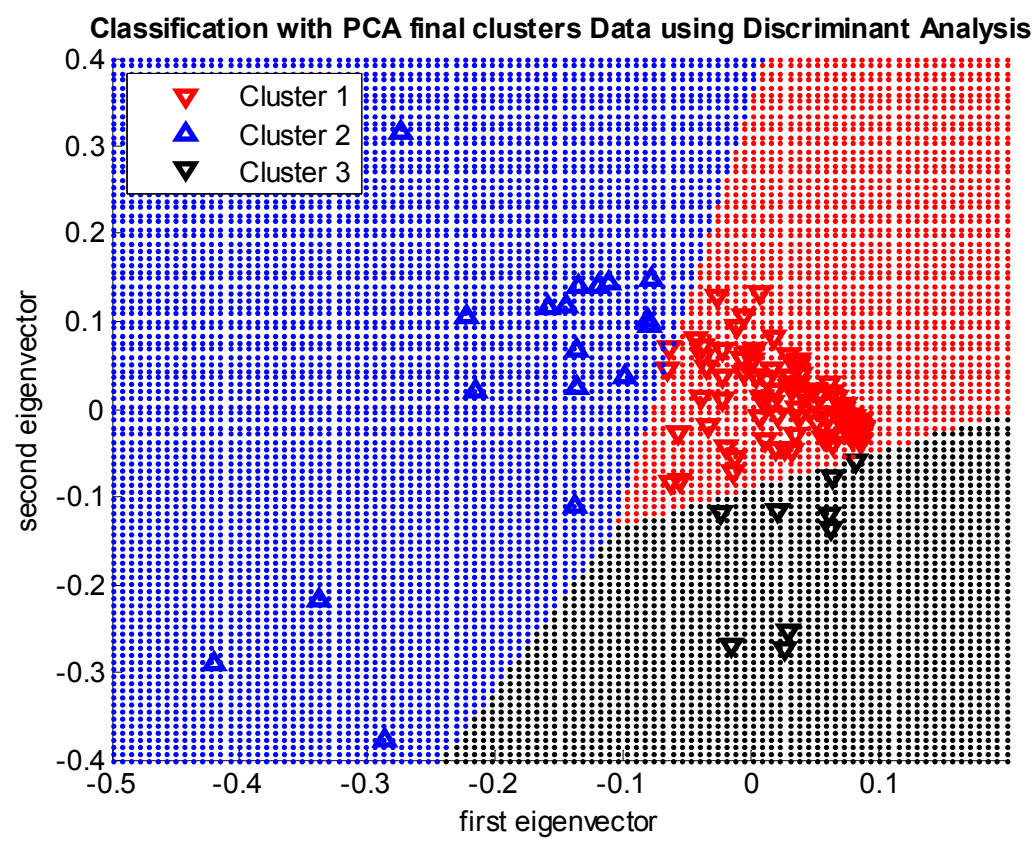

Figure 5. 7: The linear discriminant function of PCA separation results using Euclidean Distance method. 
Table 5. 7: Comparative results of second round classification using the modified Euclidean method and discriminant analysis method.

\begin{tabular}{|c|c|c|c|c|}
\hline & $\begin{array}{c}\text { Primary } \\
\text { Cluster } \\
\text { Members }\end{array}$ & $\begin{array}{c}\text { Rest Euclidean } \\
\text { Distance } \\
\text { method } \\
\text { (Dim:3-7) }\end{array}$ & $\begin{array}{c}\text { Rest } \\
\text { Discriminant } \\
\text { Analysis } \\
\text { Linear } \\
(\text { Dim:2,3) }\end{array}$ & $\begin{array}{c}\text { Rest } \\
\text { Discriminant } \\
\text { Analysis Linear } \\
\text { (Dim:4,5) }\end{array}$ \\
\hline Cluster 1 & 32 & 63 & 61 & 62 \\
\hline Cluster 2 & 10 & 8 & 8 & 8 \\
\hline Cluster 3 & 5 & 4 & 6 & 5 \\
\hline
\end{tabular}

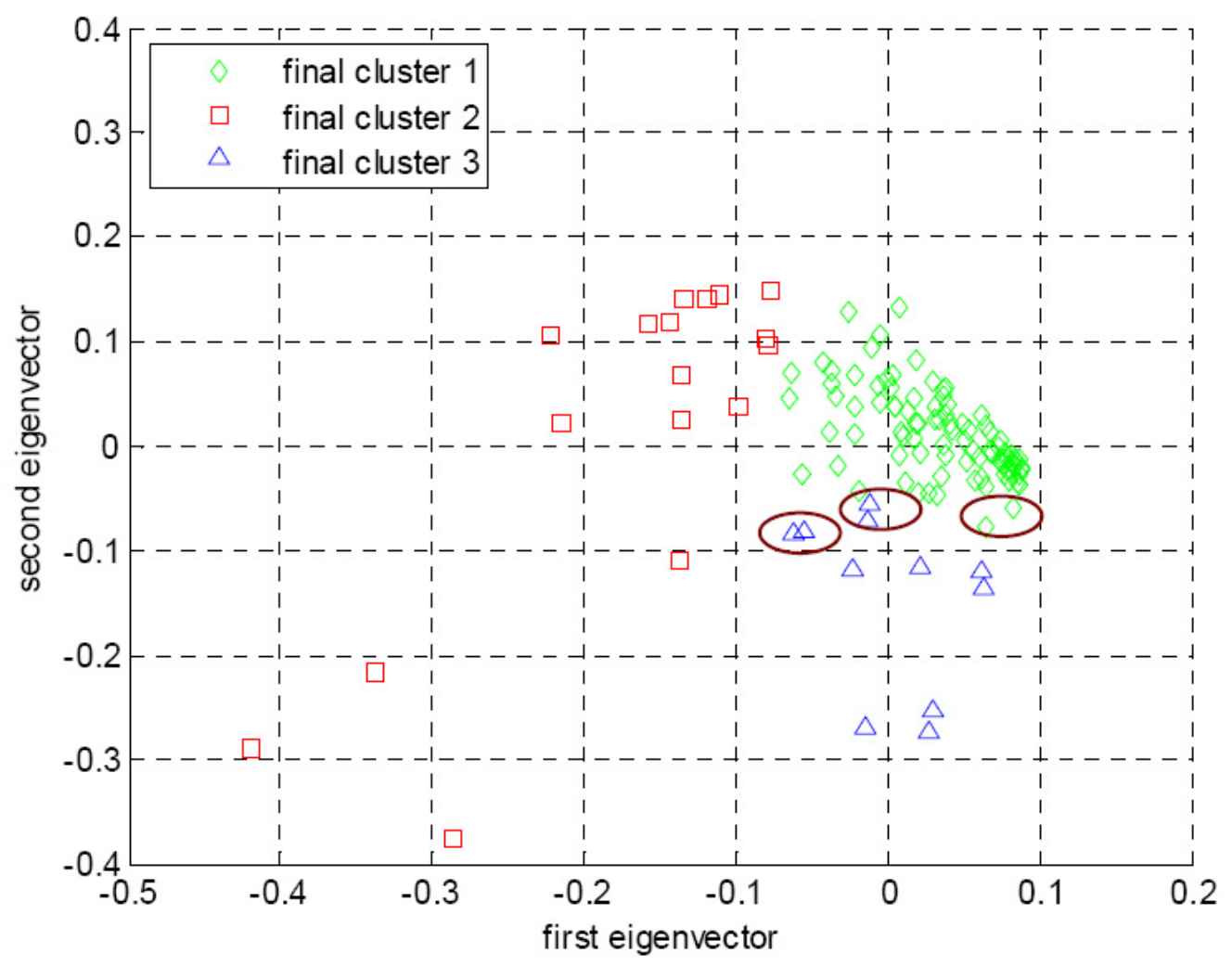

Figure 5. 8: Classification results of undecided subjects using discriminant analysis method. Note that we circled the 6 mismatch cases between discriminant Analysis Method and our PCA-Based method. 


\section{CHAPTER VI}

Sub-Patterns of Language network Reorganization in Pediatric Localization Related Epilespy

\subsection{Introduction}

This chapter focuses on the clinical relevance of the language sub-patterns found in the pediatric Localization Related Epilepsy population. Epilepsy populations provide an important window into capacity for neural plasticity as the location of essential brain functions needs to be identified for epilepsy surgery. It is known from long experience that several essential domains are perturbed by epilepsy or its underlying causes. While there are studies that have examined motor control (Muller et al., 1998a), declarative memory, and working memory networks (Dupont et al., 2000; Rabin et al., 2004; Richardson et al., 2004). Most interest has focused on language systems. Notably there is a higher incidence of atypical language dominance in epilepsy populations (Gaillard et al., 2007; Rasmussen and Milner, 1977; Thivard et al., 2005; Woermann et al., 2003). The functional anatomy of language processing networks has been extensively studied through IAT (Rasmussen and Milner, 1977), 15O-water-PET (Blank et al., 2002; Muller et al., 1998b; Petersen et al., 1988; Wise et al., 1991) and fMRI (Binder et al., 1995; Bookheimer, 2002; Cabeza and Nyberg, 2000; Just et al., 1996).

Language is typically left hemisphere dominant, but there are recognized variants (bilateral or right dominance) in normal right-handed (prevalence $=5 \%$ ) and left-handed populations (=22\%) (Pujol et al., 1999; Rasmussen and Milner, 1977; Szaflarski et al., 
2002; Woods et al., 1988). Furthermore, patients with localization related epilepsy (LRE) exhibit a higher prevalence of atypical language dominance (20-30\%). Most fMRI studies are based on visual (Fernandez et al., 2001; Gaillard et al., 2002, 2004) or ROI asymmetry indices (Binder et al.,1996; Frost et al., 1999; Gaillard et al., 2002, 2007; Ramsey et al., 2001; Spreer et al., 2002;Woermann et al., 2003) and only examine interhemispheric "reorganization." Other studies examine regional differences but also rely either on ROI asymmetry indices or regression analysis on clinical variables (Berl et al., 2006; Billingsley et al., 2001; Gaillard et al., 2007; Voets et al., 2006; Weber et al., 2006) all depending on presumptions of where language "activation" is "known" to occur based on understanding of normative data. There are ECS studies that purport to examine intrahemispheric differences (Hamberger et al., 2007; Ojemann et al., 2008), but these do not have control data and can not examine language processing outside the surgical field.

Atypical language patterns may represent: (1) "reorganization", where the primary region of language processing has moved; or, (2) "compensation", where additional areas are recruited to assist in language processing. Most commonly, studies have identified inter-hemispheric shifts to the right homologues of Broca's and Wernicke's areas Gaillard et al. (2002, 2004, 2007); Staudt et al. (2001) (Staudt et al., 2002)). Intra-hemisphere re- organization studies are less common. Using comparison of activation maxima, there is modest evidence for greater variance in temporal regions and a shift in temporal activation posteriorly and superiorly in left hemisphere seizure focus patients who remain left dominant, but they found little evidence for intra-hemispheric reorganization/compensation in patients (Rosenberger et al., 2009). Employing a PCA of difference maps between a group of normal left hemisphere dominant controls and 
individual patients with LRE, a subgroup of patients with recruitment of posterior temporal areas was also found; atypical language appeared restricted to the distributed language network homologues and margins. These findings serve as evidence for intra and inter-hemispheric language reorganization, but they couldn't distinguish between reorganization and compensation (Mbwana et al., 2009). Moreover, their results were based on the assumption of homogeneity of the analyzed population, where they were comparing epilepsy group with control group in which they pre-select the control as normal data.

However, another form of compensation, based on intensity level differences instead of location, may not be identified by current methods. This is because intensity normalization is traditionally used as a pre-processing step to scale a group of fMRI activation maps to the same intensity range. For example, sub-profile modeling (SSM) uses the natural- log transformation as the first step to standardize the raw image matrix (Alexander and Moeller, 1994).

One of the limitations of functional imaging studies is the assumptions that study populations are homogeneous and that a given paradigm will recognize single unvarying network identified by the experimental task. Clinical practice with patient populations, particularly involving language, suggests those assumptions are false. Patient populations of developmental and other disorders are also flawed by their assumption that patient populations are distinct form control populations in a uniform way. Some recent studies in executive functions in attention deficit hyperactivity disorder (ADHD) populations use regression analysis to show that there is a spectrum and that those with disease states who do better on given measures may more closely resemble controls (Vaidya et al., 2005). 
However, these studies are only able to interrogate their data where they find activation derived from limited data sets. Normal or pathological variants are lost in such approaches (Berl et al., 2006). To overcome such limitations it is necessary to examine large populations with controls and patients by a data driven means to identify variant sub patterns. This approach does not assume controls and patients are different, rather it allows that both patients and controls may be distributed across subgroups and allows for the ability to analyze subgroups based on clinical or other experimental features.

Limitations of standard approaches motivate the need to design objective methods for identifying language activation patterns. Previous methods are often constrained in their analyses either for the straightforward left-right differences, subjectivity associated with the use of visual rating and/or selection of ROI, or the use of data that lacks heterogeneity. In general, most group analyses of fMRI data sets look for "commonality" under the assumption of the homogeneity of the sample (Berl et al., 2005; Price et al., 2006). Moreover, other PCA studies have not included a large group of normal controls who may have atypical language representation (Mbwana et al., 2009).

We aimed to develop a PCA-based method to identify common and variant language activation patterns (shared) among control and epilepsy groups independent of a priori assumptions and biases inherent to ROI and visual analysis (Gaillard, 2004; Liegeois et al., 2004; Szaflarski et al., 2006). Important for our purposes, PCA provides a data driven group separation by the dynamic selection of the primary cluster members within any given population. Furthermore, the proposed PCA method does not require group normalization of the activation intensity as the first step to data classification thus avoiding the loss of a potentially important source of variance. Other segmentation 
methods, such as support vector machine and discriminant analysis, are classifier methods that are based on supervised training; previous knowledge of the data sets has to be known, and the training data sets have to be defined and labeled from the beginning. The proposed method takes a different approach in the clustering process on the basis of the PCA eigenspace. We are neither trying to categorize each subject into simple leftright dominance to replace the conventional clinical methods, nor striving to separate normal subjects from patients. Based on the distinct activation patterns identified by our data driven method, we then sought to gain insights into brain plasticity and compensation by examining the subjects in each language activation pattern by distinguishing features including control/patient designation, handedness, seizure focus location, and age of epilepsy onset.

We aimed to verify similarity of findings across sites, and establish data driven methods to reliably identify sub-patterns of language processing from pooled data.

\subsection{Method}

\subsubsection{The Clinical Aspect of the Subjects}

As mentioned in Chapter 2, the fMRI data and relevant clinical measures were stored in the data repository for central standardized processing. The distributions of scanner and institutions of these 64 control and 58 children with LRE (patient population) were included in this chapter were explained in Table 2.1 in Chapter 2. The basic demographic data is included in Table 6.1. The mean age of patients was 13.86 years (range from 4.5-19 years), with mean age seizure onset 8.23 years (range 1-18 years). 
There are 26 left localized patients, from which seventeen (65\%) had temporal focus and the rest with extra-temporal focus. There are 18 right localized patients, from which seven (39\%) had temporal focus and the rest had an extra-temporal focus. Three patients had bilateral seizure focus. Twenty two patients had abnormal MRI: seven tumor; five mesial temporal sclerosis; four focal cortical dysplasia; one vascular malfunction, three focal gliosis, and two atrophy. Of the 45 patients with seizure etiology information, 21 had remote symptomatic seizure etiology, 21 cryptogenic and 3 acute symptomatic. Eleven patients (out of the 54 available) had atypical handedness (left or ambidextrous) as determined by clinical assessment or handedness inventories such as the Harris tests of lateral dominance or the modified Edinburgh inventory (Harris, 1974; Oldfield, 1971).

Table 6. 1: Distribution of basic demographic data

\begin{tabular}{lll}
\hline & Patients & Controls \\
\hline Number & 58 & 64 \\
Male (\%) & 63.79 & 54.69 \\
Atypical handedness (\%) & 19 & 0 \\
Mean Age (years) & $13.86(4.5-19)$ & $8.65(4.2-12.9)$ \\
Mean age of seizure onset & $8.23(1-18)$ & - \\
Temporal focus of Left localized (\%) & 65 & - \\
Temporal focus of Right localized (\%) & 39 & - \\
Mean duration of seizures (min) & 2.88 & -
\end{tabular}

\subsubsection{PCA-based decisional space separation}

As mentioned in Chapter 5, according to the concept and merit of subject loading, and the relationship among the top eigenvectors, general lateralization, and intensity difference, as well as the dendrogram of the Euclidian distance matrix of the PCA, 
criteria were decided for the top two eigenvectors of the PCA-based decisional space which identified three primary clusters (the first as major group left dominant, the second featured higher intensity levels, and the third with right dominant activation). Three sub-groups are identified (You et al., 2009).

\subsubsection{Group map and Significance map}

In order to verify and understand the separation results of PCA, the range and location of group member variability were assessed with the mean group map. A significance map for each group was generated. This map is different than the collective penetrance maps used by others (Mbwana et al., 2009; Seghier et al., 2008), as we sought the commonality contribution of each subject to the mean map. Based on the histogram of each mean group map, a mask containing $90 \%$ of the activation energy was defined. The group significance map is then computed by first masking each individual activation map (within each group), then calculating the commonality significance value as defined in Eq.6.1.

$$
C s=e^{-\frac{\left(\text { Value }_{\text {voxel }}-\text { Mean }\right)^{2}}{2 S D^{2}}}
$$

The Commonality significance (Cs) value is calculated for each voxel within the masked area, and then the total group significance map is generated by averaging the Cs values across the subjects within a given group. This provides a visual representation of the areas that have a significant percentage of subjects sharing the same location of activation.

\subsubsection{Statistical Analysis}


The association of clinical factors with the group distribution was analyzed using either Fisher exact test for categorical data or ANOVA and t-test for continuous data. If the overall Fisher exact test was significant, pairwise comparisons of groups were performed. The Holm's sequential Bonferroni procedure was then applied to correct for the probability of a Type I error (alpha $=0.05$ ).

\subsection{Results}

\subsubsection{Activation patterns and significance maps}

The PCA analysis identified three distinct groups of subjects after the selfseparation process utilizing the top subject loadings and distance method. The activated areas of the three group activation patterns broadly encompass Broca's and Wernicke's areas. Group 1 exhibited activation in the left hemisphere (Figure 6.1.a and Table 6.2). Group 2 (Figure 6.1.b) consisted of a cluster of subjects that shared the same general activation areas as group 1; however, the magnitude of activation for group 2 was stronger than those of group 1, especially in Broca's area, as shown in Figure 6.1.b and Table 6.2, and additional activation was evident in left MFG (BA 46, 9), left SMA (BA 6), and right cerebellum. Group 3 had activation in right hemisphere homologues (Figure 6.1.c and Table 6.2). The distribution of patients and controls differed among the three groups ( $\mathrm{p}<0.0004)$. Group 1 consisted of nearly all the healthy controls and a majority of patients; groups 2 and 3 were composed principally of patients but included a few typically developing controls. In terms of typical language activation, LRE patients had 
greater magnitude of activation than controls based on the subjects distribution in groups

1 and 2 (Fisher exact test; $p=0.0005)$.

In order to appraise the subjects' contribution for each group map, a group significance map was generated for each group as shown in Figure 6.2. This figure helps to visualize the variance of the separation results comparing the group members with the group map. The maximum commonality significance value for the three groups are higher than 0.8; group 1 has the least variance and group 3 has the most variance.

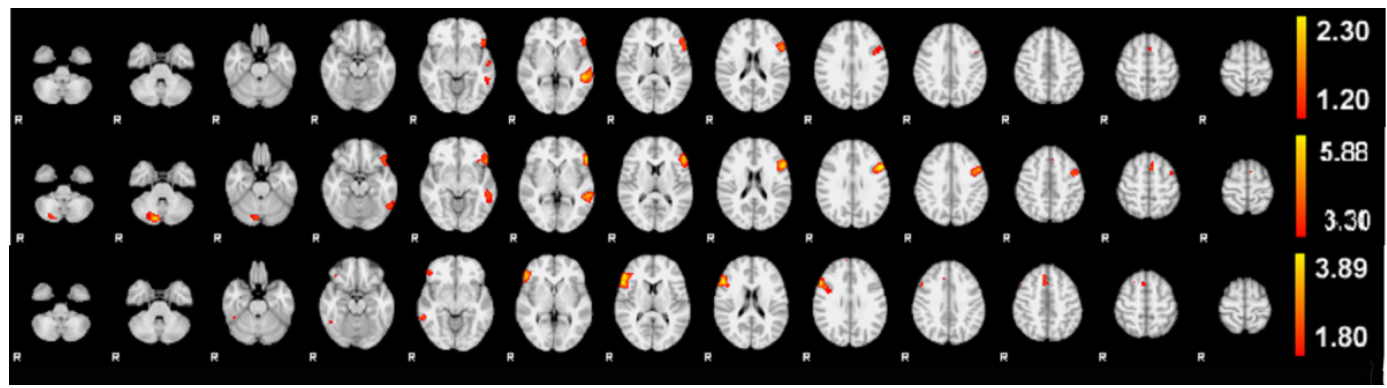

Figure 6. 1:2D array of selected axial cuts of the mean activation maps for each group overlaid on the MNI standard brain. Higher activations are in yellow color. Brain is oriented in radiological convention: right hemisphere on the left side. (a) Mean activation map for group 1 with strong left lateralization of anterior (Broca) and posterior (Wernicke) clusters. (b) Mean activation map for group 2 with higher mean intensity range than (a), which explains the better definition of Supplementary Motor Area (SMA). (c) Mean activation map for group 3 with an atypical right hemisphere dominant response, particularly the anterior (Broca) cluster. Different intensity threshold ( $90 \%$ of the energy) was used for visualization purpose.

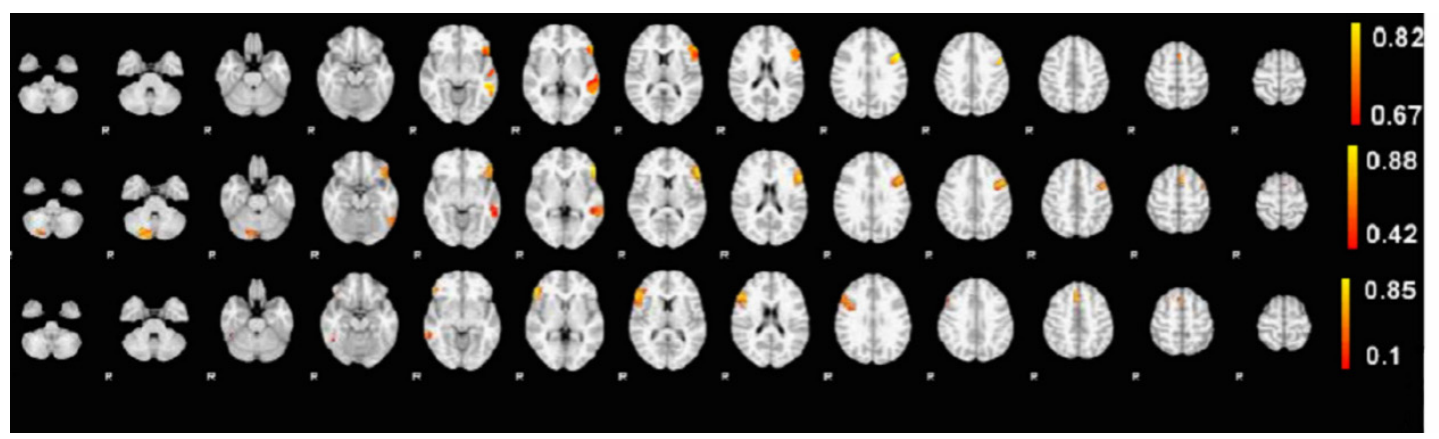

(a)

Figure 6. 2: Commonality significance map of each group. All three groups have the highest significance value higher than 0.8 and group 1 (a) has the least variance among the group members in the activated area, while group 3 (c) has the largest variance. 
Table 6.2 provides the mean map's activation maxima of each small cluster within each group and their coordinates, cluster size, the peak value of each cluster, and corresponding commonality significance value, and corresponding Brodmann Area. Moreover, a second level t-test was performed comparing the mean map of group 1 to group 2; Figure 6.3 depicts the areas that remain significantly different.

Table 6. 2: Activation location, size, peak values and commonality significance value for each group map*

\begin{tabular}{|c|c|c|c|c|c|}
\hline Group & Cluster size & Mean-Z (peak) & Cs of the peak & $\begin{array}{c}\mathrm{x}, \mathrm{y}, \mathrm{z} \\
\text { (Voxel Space }+ \text { ) }\end{array}$ & Region (BA) \\
\hline \multirow[t]{3}{*}{1} & 319 & 1.91 & 0.74 & $\begin{array}{lll}48 & 47 & 31\end{array}$ & LIFG (44) \\
\hline & 248 & 2.3 & 0.74 & $29 \quad 24$ & LMTG (21) \\
\hline & 10 & 1.42 & 0.76 & 47 & RIFG (32) \\
\hline \multirow[t]{4}{*}{2} & 1014 & 5.88 & 0.80 & $47 \quad 32$ & LIFG (44/45) \\
\hline & 416 & 5.2 & 0.68 & 2923 & LMTG (21) \\
\hline & 338 & 5.24 & 0.73 & 1512 & $\mathrm{R}$ cerebellum \\
\hline & 147 & 4.26 & 0.72 & $46 \quad 42$ & RMFG (46) \\
\hline \multirow[t]{3}{*}{3} & 500 & 3.89 & 0.66 & 5028 & RIFG (45/48) \\
\hline & 61 & 2.51 & 0.71 & 5240 & RMTG (8) \\
\hline & 35 & 2.78 & 0.46 & $27 \quad 22$ & RMFG $(37 / 20)$ \\
\hline
\end{tabular}

* The cluster size here reflects the number of thresholded voxels within the cluster of the mean activation map. Threshold values are 1.2 for group 1, 3.3 for group 2, 1.8 for group 3, same as the threshold used for visualization purpose in Fig.1, containing 90\% of the activation energy. The largest cluster in group 2 has a maxima in IFG but extends into left MFG. + The Voxel Space we use here is the FSL MNI space, using coordinates as: $\mathrm{x}$-axis as the right-left direction (moving in the left direction increases the $\mathrm{x}$ voxel index, range 1-61); $y$-axis as the posterior-anterior direction (moving in the anterior direction increases the y voxel index, range 1-73); z-axis as the inferior-superior direction (moving in the superior direction increases the $\mathrm{z}$ voxel index, range 1-61).

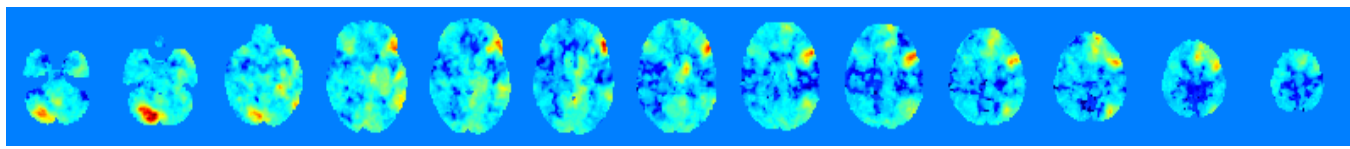

Figure 6. 3: Second level t-test comparing the mean map of group 1 to group 2. Note the high $t$ values (significant level $\mathrm{p}<0.01$ ) in the shared activated area, which is in the left IFG and MFG.

The cluster size here reflects the number of thresholded voxels within the cluster of the mean activation map. Threshold values are 1.2 for group 1, 3.3 for group 2, 1.8 for group 3, same as the threshold used for visualization purpose in Figure 6.1, containing 
$90 \%$ of the activation energy. The largest cluster in group 2 has a maxima in IFG but extends into left MFG. Coordinates in voxel space: $\mathrm{x}$-axis as the right-left direction (moving in the left direction increases the $\mathrm{x}$ voxel index, range 1-61); $\mathrm{y}$-axis as the posterior-anterior direction (moving in the anterior direction increases the y voxel index, range 1-73); z-axis as the inferior-superior direction (moving in the superior direction increases the $z$ voxel index, range 1-61).

\subsubsection{Demographic and clinical variables}

We found no difference in age at seizure onset, duration of epilepsy and gender between the three groups. However, there was an age difference among the three groups [ANOVA, $\mathrm{F}(2, \mathrm{n}=118)=9.44, \mathrm{p}=0.0002$ ]; differences were found between groups 1 and $2(\mathrm{~F}=3.78, \mathrm{p}=0.001$, Bonferroni), as well as between group 1 and $3(\mathrm{~F}=3.16, \mathrm{p}=0.05$, Bonferroni). Group 1 was younger than group $2[\mathrm{t}(108, \mathrm{n}=110)=-3.91, \mathrm{p}=0.002]$.

Table 6.3 and Figure 6.4 present the patient's group profiles with related categorical variables and illustrate the clinical factors distribution among these three groups. There were no differences based on gender seizure focus and etiology among the three groups. Data from groups 1 and 2 were compared first, since both groups were left lateralized but exhibited different intensities. The distribution of seizure focus between groups 1 and 2 are different $\left[\left(\mathrm{X}^{2}=13, \mathrm{n}=50\right)=21.731, \mathrm{p}=0.03\right]$; the patients of group 2 had a higher percentage ( $50 \%$ to $34 \%$ ) in terms of right seizure focus. In contrast, group 3 with right activation was largely male (6 out of 8 ), left handed (5 out of 8), with a left seizure focus (6 out of 8), and had a history of (poorly controlled) symptomatic LRE (6). Patients' data were then compared between group 1 and group 3. Patients in group 3 had 
a higher percentage of left seizure focus than in group $1(71.4 \%$ vs. $53 \%)$; the handedness distribution is also different from group 1 (Fisher exact test, $\mathrm{p}=0.007$; Table 6.4). The other clinical variables - age, gender, age of onset, and seizure duration - were not different between these two groups. Data were then compared between the two broad groups, left lateralized (group 1+2) and right lateralized (group 3); the handedness difference was significant (Fisher exact test, $\mathrm{p}=0.003$ ) and left handed patients tended to have right hemisphere activation (group 3, Fisher exact test, $\mathrm{p}=0.002$; Table 6.4). No significant difference of seizure etiology or seizure focus was found between these two broad groups.

Table 6. 3: Profile of clinical factors of three groups divided by PCA method

\begin{tabular}{cl|ccc}
\hline \multirow{5}{*}{ Clinical factors } & PCA Groups & 1 & 2 & 3 \\
& Ambidextrous & 2 & 0 & 0 \\
& Right & 27 & 13 & 3 \\
& Left & 3 & 1 & 5 \\
& N/A & 3 & 1 & 0 \\
& Total & 35 & 15 & 8 \\
\hline \multirow{5}{*}{ Seizure focus } & Bilateral & 3 & 0 & 0 \\
& Right & 9 & 7 & 2 \\
& Left & 14 & 7 & 5 \\
& N/A & 9 & 1 & 1 \\
& Total & 35 & 15 & 8 \\
\hline \multirow{5}{*}{ Etiology } & Acute & 1 & 1 & 1 \\
& Cryptogenic & 11 & 7 & 3 \\
& Remote & 15 & 3 & 3 \\
& Symptomatic & & 4 & 1 \\
& N/A & 8 & 15 & 8 \\
\hline \multirow{5}{*}{ Gender } & Total & 35 & 8 & 6 \\
& Male & 23 & 7 & 2 \\
& Female & 12 & 15 & 8
\end{tabular}

*Fisher exact test, comparison among group 1-3, $p=0.007(p<0.0167$ Holm's sequential Bonferroni correction). Holm's sequential Bonferroni correction procedure: Since the overall difference among the three groups is significant in handedness (Fisher exact test, $p=0.0079$ ), now comparing the smallest $\mathrm{p}$ value first, which is between group 1-3 $p=0.007<0.05 /$, so it's significant; now compare the second smallest one between group 2-3, $p=0.02<0.05 / 2$, still significant; but the third significant $p$ value between group $1-2,0.6$ is not significant. 


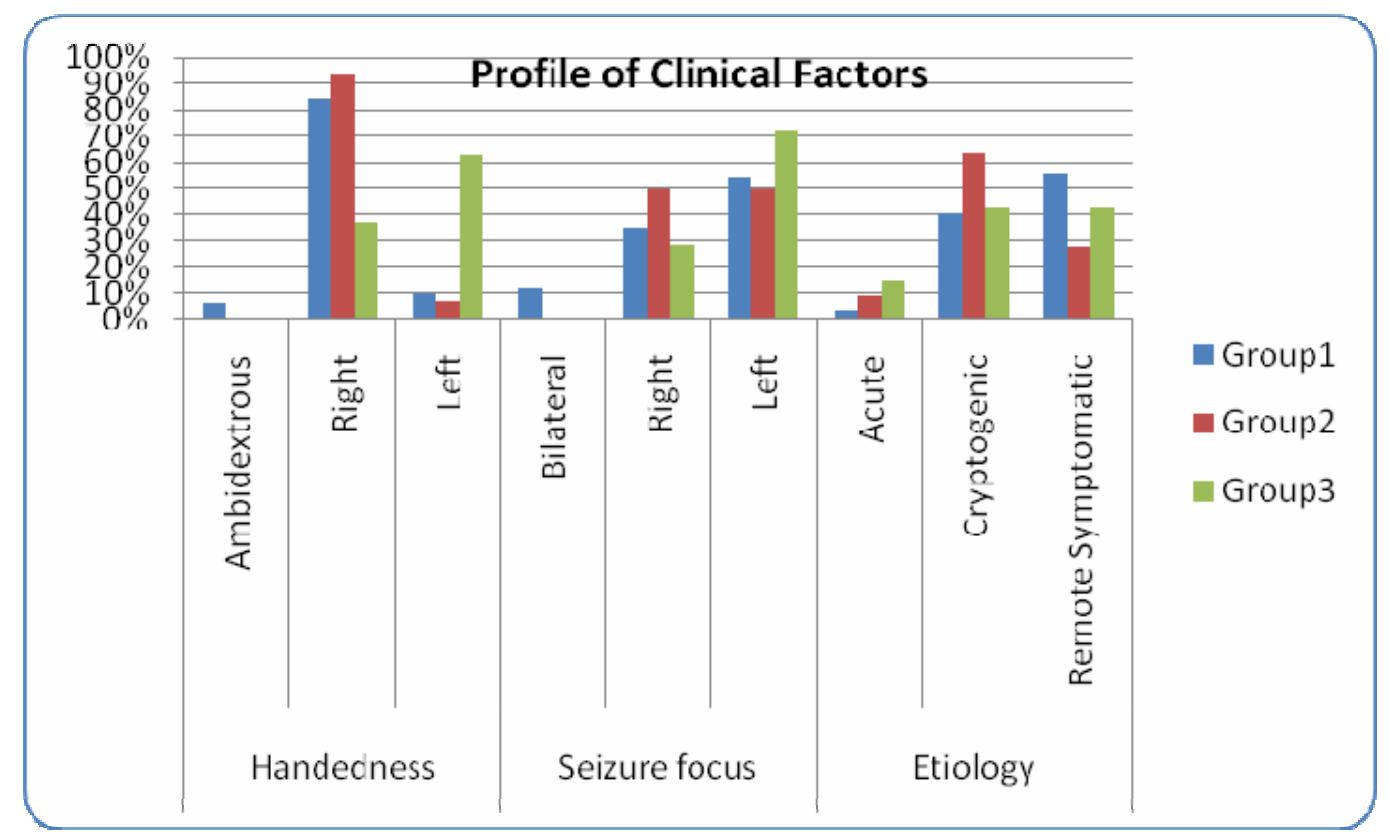

Figure 6. 4: Clinical Factor distribution among three groups. The percentage of patients in each group based on handedness, seizure focus and seizure etiology findings. Handedness was different among the three groups, and between group 1 vs. group 3, and between group $(1+2)$ vs. group 3. $(\mathrm{p}<0.0167$ Holm's sequential Bonferroni correction).

Table 6.4 : Distribution of handedness across three groups with regard to seizure focus *

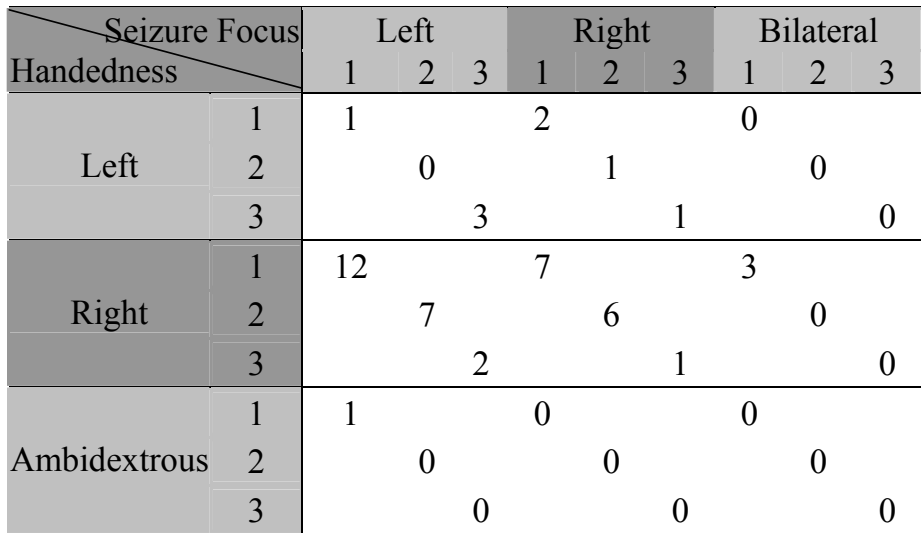

* Only 47 data sets combined the information on seizure focus and handedness. Notice the numbers are too few in some subgroups to make statistical comparisons meaningful. 


\subsection{Discussion}

We used the new method of PCA-based decisional space as described in Chapter 5 to identify distinct language activation patterns in control and LRE patients from different sites, who performed the same fMRI auditory description decision task. Three sub-groups were identified: two with predominantly left hemispheric activation but with different regional weighting of activity, and one with a predominantly right-sided activation pattern. Normal controls and patients fell into each of the three groups but the distribution differed with a greater proportion of controls in the first and largest left activation group while patients were the majority in the other two groups. Unlike ROI analysis employed to generate an asymmetry index, our method did not provide determination of language dominance, but aimed to identify distinct activation patterns . These findings provide insight into reorganization of language system functions and potential compensatory strategies in epilepsy and normal populations.

Our findings suggest variants of language patterns which are not revealed in previous studies (group 2); secondary analysis suggests the variant patterns are more common to epilepsy patients than to controls. Our methods sorted subjects by imaging features independent of whether a child had epilepsy or was a control. The broad distinction of left and right hemisphere dominant patterns identified in our study are similar to prior studies on language dominance in normal volunteers and in epilepsy populations employing transcranial-Doppler, transcranial magnetic stimulation, the IAT, and conventional fMRI analysis (Binder et al., 1996; Fernandez et al., 2001; Gaillard et

al., 2002; Khedr et al., 2002; Knecht et al., 2000; Kurthen et al., 1994; Rasmussen and Milner, 1977; Risse et al., 1997; Woods et al., 1988; Wyllie et al., 1991). The right 
language group (Group 3), contained $7 \%$ of the total population and $14 \%$ of the LRE population, which is comparable to previous typically developing and epilepsy patient studies. The majority of patients in this group had left seizure focus, was left-handed, and had left structural lesions, all factors known to be associated with atypical language dominance (Gaillard et al., 2007; Woermann et al., 2003). While activation in this group occurred in the right hemisphere in areas that mirror activation seen in the lefthemisphere patterns (Gaillard et al., 2002; Mbwana et al., 2009; Rosenberger et al., 2009; Staudt et al., 2001) - this group also showed the greatest variance. Some studies suggest that atypical language dominance in patient populations is tightly constrained to right homologues (Rosenberger et al., 2009; Staudt et al., 2001) but others suggest greater variability when language has shifted to the typically non-dominant hemisphere (Voets et al., 2006). These patterns are considered to represent "reorganization" from the left to the right hemisphere in response to epilepsy or its remote cause (Gaillard et al., 2007; Mbwana et al., 2009). Findings in this study suggest that transfer of language dominance across hemispheres may be imperfect in some patients.

Intra-hemispheric variants, however, have been harder to identify by conventional analytic approaches. We identified two groups with left hemisphere patterns of activation. The larger group (group 1) is composed of nearly all typically developing children and the majority of patients. We also identified another group (group 2), composed of mostly patients and a minority of typically developing controls. This group had a different left hemisphere activation pattern than the first group that involved different activation intensity in the inferior frontal regions and recruitment of adjacent MFG (BA 46, 9), SMA (BA 6) and contralateral cerebellum. The regions observed are all 
areas identified with the widely distributed left hemisphere language processing network but are also those thought to be engaged in verbal working memory (Baillieux et al., 2008; Stoodley and Schmahmann, 2009). In addition, these subjects express the highest measure of commonality, that is, the least variance in the IFG (BA 44/45).

This data suggests tighter homogeneity of activation in this group than in the others. There are two possible explanations for these findings. Activation in these areas may reflect greater engagement of verbal working memory systems, possibly due to effort, perceived difficulty, effect of medications, effect of epilepsy, or compensation for impaired hippocampal memory function (Berl et al., 2005; Dupont et al., 2000). Turkeltaub et al. (2003 \& 2004) found reading-skill acquisition for young readers is associated with increasing activity in the left inferior frontal gyrus and middle temporal gyrus and decreasing activity in the right extrastriate cortex. Moreover, they also found increased covert reading activity for the hyperlexic subject compared to control subjects in reading-related areas Turkeltaub et al. $(2003,2004)$. These cases support the idea that intensity has a meaningful role in task processing; when intensity of a region is greater than what one would expect, it suggests a differential recruitment of neural networks in that region for that task.

Group 2 also had a higher percentage of patients with a right seizure focus. A right seizure focus may compromise ancillary and non linguistic aspects of language processing that occurs in the right hemisphere, requiring compensation in the left hemisphere (Berl et al., 2005). In this view, the group 2 left activation pattern represents compensation rather than reorganization (Berl et al., 2005; Mbwana et al., 2009) and suggests a possible remote effect on of a right hemisphere focus on traditionally left- 
lateralized functions. These patients may draw upon the distributed language network in a different way than most controls.

Moreover, there also exists the possibility of modulation of the nodes of a bigger network in processing language via changes in functional connectivity, which might be examined with other methods such as connectivity analysis (Hampson et al., 2002). The functional connectivity may serve as a possible alternative mechanism to which our method is not tuned to detect.

Some of the differences that characterize group 3 may represent an effect of handedness. None of our typically developing children were left handed or ambidextrous. However, previous studies involving left handed controls (and it is not clear how many had acquired sinistrality) show that $76-78 \%$ are left dominant (Pujol et al., 1999; Szaflarski et al., 2002). Moreover, left handed patients are over represented in epilepsy populations; $56 \%$ or more of left handed patients may be expected to have atypical language dominance - more than left handed controls (Gaillard et al., 2007; Rasmussen and Milner, 1977). These data suggest that both atypical language dominance and atypical handedness are reflections of the underlying epilepsy or its remote cause.

A substantial study population enhances the ability to identify variant patterns of language networks by data driven methods and gain insight into the neurobiology of complicated cognitive processes. Larger populations of cooperatively acquired data will allow for identification of additional, less common, variants and meaningful interpretation of these patterns based on clinical characteristics, such as seizure focus and pathological substrate, among others. This information is necessary to improve care and outcomes. The PCA-decisional space presented here can be helpful in sorting an 
individual patient into a particular language pattern subset without the bias and limitations inherent to the traditional fMRI patient care analysis. The proposed method might also be useful for assessing large combined patient and control data sets in which visual or ROI rating may be impractical or difficult. This is especially applicable for those developmental disorders where population differences are not readily apparent and assumptions of patient population homogeneity are unrealistic. For epilepsy, future research may also take advantage of the PCA-decisional space characteristics for group separation in order to overcome conceptual limitations of language network organization that categorize into simple patterns of left, bilateral or right dominance.

Not applying intensity normalization allowed us to consider simultaneously the location, extent, and magnitude of activation intensity; this method helped identify a subgroup with a left hemisphere activation pattern distinct form one more commonly found in normal controls and in the majority of patients. We also introduced a significance map derived from the subgroup and further analyzed the segregation results by clinical variables. Our analysis supports the notion of pooled data from several institutions using the same paradigm and comparable acquisition parameters. We do not claim that our method is better than other segregation methods, rather we suggest that this or similar methods applied to substantial normal, developmental and patient populations may identify normal and pathological activation patterns for cognitive systems that in turn provide insights into mechanisms for brain compensation and neural plasticity. 


\title{
CHAPTER VII
}

\author{
Conclusions
}

The main objective of this dissertation is to develop integrated methods to assess and automatically classify different language network activation patterns in a mixed fMRI population containing both control and LRE patients. A novel approach is thus proposed in which the PCA is seamlessly combined with generalized nonlinear classifiers and the Euclidean distance method. The LI and clinical rating are used as support mechanisms to the decisional space to gauge the accuracy of the automated decision making process. In the early phase of this research, a multisite image consortium was developed for pediatric epilepsy, which provided a fast, reliable, fault tolerant, secure environment that brought several hospitals with renowned pediatric epilepsy programs together.

The heterogeneity in the patterns of typical versus atypical brain organization is such that no one center can identify a sufficient number of patients to investigate the contribution of the different factors that influence brain organization and underlying pathologies. For this singular reason, the consortium that was built here at FIU was structured to not only promote objective assessments of large data sets that can serve to interrogate data for a multitude of clinical variables, but also to elicit a better understanding of the brain language network plasticity in a mixed pediatric epilepsy and control population. Thus, the conscientious decision not to perform intensity normalization during the second level analysis on the activation maps granted the 
opportunity to discover activation intensity compensation for language networks among some of the subjects.

Groups 1 and 2 were identified as left dominant subjects in reference to their language dominance, but they were segregated due to the intensity difference presented by their activation patterns. Group 3 was represented by right dominance subjects as expected; however, group 2 was an unexpected finding.

The further advantage is that one can work backwards along this process to identify clinical features that characterize these groups. One of the distinguishing factors is that patients and controls are found in all three groups. This is an important finding, one that we have emphasized from the outset for not focusing solely on segregating control from patients. The control and patients are unequally represented, raising questions about what the variants mean. There are very few studies that have done what we have accomplished with this large mixed population.

The PCA-decisional space as designed is proactive for assessing large combined patient and control data sets in which visual rating or ROI-based LI may be impractical or difficult. The way of direct implementation on a mixed population is especially applicable for those developmental disorders where population differences are not readily apparent, and assumptions of patient population homogeneity are unrealistic. For epilepsy, future research may also take advantage of the PCA-decisional space characteristics for group separation in order to overcome conceptual limitations of language network organization that categorize into simple patterns of left, bilateral or right dominance. 
e space in order to optimize the classification of fMRI language related activation brain patterns. This integration resulted in a datadriven method that is both accurate and computationally appealing.

2. Devised an automated method to perform objective classification of brain activation patterns as a support tool to assist the more subjective visual rating methods or ROI-based LI methods, but with no intent to substitute them. The sensitivity and objectivity of the PCA-NDF for group separation helps in overcoming human rating error as well as the limitations imposed by prior assumptions.

3. Introduced the idea of using synthetic activation patterns, derived randomly from real data, to help our method perform classification on language activation patterns into typical and atypical categories. The data-driven mechanism using NDF was found to be effective at classifying typical and atypical language networks activation patterns from a heterogeneous population often acquired with different acquisition parameters. Recall that the optimal nonlinear classifier yielded results with 96\% accuracy, 97\% sensitivity, 95\% specificity, and 95\% precision.

4. Established a PCA-based decisional space to recognize groups of distinct language activation patterns in a mixed population of control and LRE patients from different sites. In this data driven method, mathematical thresholds were uniquely derived to delineate regions for three primary clusters based on the first 
two eigenvectors of the PCA; while the modified-Euclidean distance method is used to classify any undecided subjects into one of the three primary clusters initially determined.

5. Avoided intensity normalization to allow for the simultaneous consideration of the location, extent, and magnitude of activation intensity; this method helped identify a group with a left hemisphere activation pattern distinct form one more commonly found in normal controls and in the majority of patients.

6. Introduced a significance map derived from the subgroup and further analyzed the segregation results by clinical variables. The two left dominant subgroups differed on the intensity level of regional activations. Some of the findings may represent an effect of epilepsy, its underlying substrate on language network expression, or may represent different strategies in performing the task. The results showed good agreement with both the subjective visual categorization and the ROI-based LI distribution in identifying the strong right dominant group.

7. Provided standardization procedures to account for different data formats and site differences such as scanner manufacturer, magnetic strength and acquisition parameters, which are perceived as limitations that hinder group analysis. Our analysis showed that our clustering results were independent of scanner or site.

It is worth re-emphasizing that the methods developed in this dissertation promote objective assessments of large data sets which can consequently allow doctors and clinicians to interrogate data for a multitude of clinical variables that otherwise would be too unyielding to manage. Our analysis supports the notion of pooled data from several institutions using the same paradigm and comparable acquisition parameters. However, 
we do not claim that the proposed method is better than other segregation methods; rather we suggest that these methods applied to developmental populations may identify normal and pathological activation patterns for cognitive systems. In the future, some efforts should be invested in extending our method into other paradigms that are available in our multisite consortium, such as the auditory category task and listening task, in order to advance our understanding of the language networks. Moreover, the method as designed provides insights into mechanisms for brain compensation and neural plasticity, which can be extended to study other brain abnormalities such as Schizophrenia, Alzheimer and autism. 


\section{LIST OF REFERENCES}

Abramoff, M., Magalhaes, P., Ram, S., 2004. Image processing with ImageJ. Biophotonics International 11 (7), 36-43.

Alexander, G., Moeller, J., 1994. Application of the scaled subprofile model to functional imaging in neuropsychiatric disorders: a principal component approach to modeling brain function in disease. Human Brain Mapping 2 (1-2), 79 - 94.

Andersen, A. H., Gash, D. M., Avison, M. J., 1999. Principal component analysis of the dynamic response measured by fMRI: a generalized linear systems framework. Magn Reson Imaging 17 (6), 795-815.

Baillieux, H., De Smet, H. J., Paquier, P. F., De Deyn, P. P., Marien, P., 2008. Cerebellar neurocognition: insights into the bottom of the brain. Clin Neurol Neurosurg 110 (8), $763-73$.

Beckmann, C., Smith, S., 2004. Probabilistic independent component analysis for functional magnetic resonance imaging. IEEE transactions on medical imaging 23 (2), $137-152$.

Berl, M. M., Balsamo, L. M., Xu, B., Moore, E. N., Weinstein, S. L., Conry, J. A., Pearl, P. L., Sachs, B. C., Grandin, C. B., Frattali, C., Ritter, F. J., Sato, S., Theodore, W. H., Gaillard, W. D., 2005. Seizure focus affects regional language networks assessed by fMRI. Neurology 65 (10), 1604-1611.

Berl, M. M., Vaidya, C. J., Gaillard, W. D., 2006. Functional imaging of developmental and adaptive changes in neurocognition. Neuroimage 30 (3), 679-91.

Billingsley, R., McAndrews, M., Crawley, A., Mikulis, D., 2001. Functional mri of phonological and semantic processing in temporal lobe epilepsy. Brain 124 (6), 1218.

Binder, J. R., Rao, S. M., Hammeke, T. A., Frost, J. A., Bandettini, P. A., Jesmanowicz, A., Hyde, J. S., 1995. Lateralized human brain language systems demonstrated by task subtraction functional magnetic resonance imaging. Arch Neurol 52 (6), 593-601.

Binder, J. R., Swanson, S. J., Hammeke, T. A., Morris, G. L., Mueller, W. M., Fischer, M., Benbadis, S., Frost, J. A., Rao, S. M., Haughton, V. M., 1996. Determination of language dominance using functional mri: a comparison with the wada test. Neurology 46 (4), 978-84.

Blank, S. C., Scott, S. K., Murphy, K., Warburton, E., Wise, R. J., 2002. Speech production: Wernicke, broca and beyond. Brain 125 (Pt 8), 1829-38.

Bookheimer, S. Y., 2002. Functional mri of language: New approaches to understanding the cortical organization of semantic processing. Annual Review of Neuroscience 25 (1), 151-188. 
Boser, B., Guyon, I., Vapnik, V., 1992. A training algorithm for optimal margin classifiers. In: Proceedings of the fifth annual workshop on Computational learning theory. ACM New York, NY, USA, pp. 144-152.

Burges, C., 1998. A tutorial on support vector machines for pattern recognition. Data mining and knowledge discovery 2 (2), 121-167.

Cabeza, R., Nyberg, L., 2000. Imaging cognition ii: An empirical review of 275 pet and fMRI studies. Journal of Cognitive Neuroscience 12 (1), 1-47.

Caprihan, A., Pearlson, G. D., Calhoun, V. D., 2008. Application of principal component analysis to distinguish patients with schizophrenia from healthy controls based on fractional anisotropy measurements. Neuroimage 42 (2), 675-82.

Carroll, J., Davies, P., Richman, B., 1971. The American heritage word frequency book. Houghton Mifflin, Boston, MA.

Cristianini, N., Shawe-Taylor, J., 2000. An introduction to support vector machines and other kernel-based learning methods. University of Cambridge Press.

Duda, R. O., Hart, P. E., Stork, D. G., 2001. Pattern Classification. John Wiley \& Sons, New York.

Dupont, S., Van de Moortele, P. F., Samson, S., Hasboun, D., Poline, J. B., Adam, C., Lehericy, S., Le Bihan, D., Samson, Y., Baulac, M., 2000. Episodic memory in left temporal lobe epilepsy: a functional mri study. Brain 123 (Pt 8), 1722-32.

Fernandez, G., de Greiff, A., von Oertzen, J., Reuber, M., Lun, S., Klaver, P., Ruhlmann, J., Reul, J., Elger, C. E., 2001. Language mapping in less than 15 minutes: real-time functional MRI during routine clinical investigation. Neuroimage 14 (3), 585-94.

Fleiss, J., 1971. Measuring nominal scale agreement among many raters. Psychological Bulletin 76 (5), 378-382.

Ford, J., Farid, H., Makedon, F., Flashman, L., McAllister, T. W., Megalooikonomou, V., Saykin, A. J., 2003. Patient classification of fmri activation maps. In: 6th Annual International Conference on Medical Image Computing and Computer Assisted Intervention. Vol. 2879. pp. 58-65.

Forman, S., Cohen, J., Fitzgerald, M., Eddy, W., Mintun, M., Noll, D., 1995. Improved assessment of significant activation in functional magnetic resonance imaging (fMRI): use of a cluster-size threshold. Magnetic Resonance in Medicine 33 (5), 636-647.

Friston, K., Worsley, K., Frackowiak, R., Mazziotta, J., Evans, A., 1994. Assessing the significance of focal activations using their spatial extent. Human Brain Mapping 1 (3), 210-220. 
Friston, K. J., Frith, C. D., Liddle, P. F., Frackowiak, R. S., 1993. Functional connectivity: the principal-component analysis of large (pet) data sets. J Cereb Blood Flow Metab13 (1), 5-14.

Frost, J. A., Binder, J. R., Springer, J. A., Hammeke, T. A., Bellgowan, P. S., Rao, S. M.,Cox, R. W., 1999. Language processing is strongly left lateralized in both sexes, evidence from functional mri. Brain 122 (Pt 2), 199-208.

Gaillard, W. D., 2004. Functional mr imaging of language, memory, and sensorimotor cortex. Neuroimaging Clinics of North America 14 (3), 471-85.

Gaillard, W. D., Balsamo, L., Xu, B., Grandin, C. B., Braniecki, S. H., Papero, P. H., Weinstein, S., Conry, J., Pearl, P. L., Sachs, B., Sato, S., Jabbari, B., Vezina, L. G., Frattali, C., Theodore, W. H., 2002. Language dominance in partial epilepsy patients identified with an fMRI reading task. Neurology 59 (2), 256-65.

Gaillard, W. D., Balsamo, L., Xu, B., McKinney, C., Papero, P. H., Weinstein, S., Conry, J., Pearl, P. L., Sachs, B., Sato, S., Vezina, L. G., Frattali, C., Theodore, W. H., 2004. fMRI language task panel improves determination of language dominance. Neurology $63(8), 1403-8$.

Gaillard, W. D., Berl, M. M., Moore, E. N., Ritzl, E. K., Rosenberger, L. R., Weinstein, S. L., Conry, J. A., Pearl, P. L., Ritter, F. F., Sato, S., Vezina, L. G., Vaidya, C. J.,Wiggs, E., Fratalli, C., Risse, G., Ratner, N. B., Gioia, G., Theodore, W. H., 2007. Atypical language in lesional and nonlesional complex partial epilepsy. Neurology 69 (18), 1761-71.

Gaillard, W. D., Sachs, B. C., Whitnah, J. R., Ahmad, Z., Balsamo, L. M., Petrella, J. R., Braniecki, S. H., McKinney, C. M., Hunter, K., Xu, B., Grandin, C. B., 2003. Developmental aspects of language processing: fmri of verbal fluency in children and adults. Human Brain Mapping 18 (3), 176-85.

Guillen, M., 2008. Design and Implementation of a Multisite Data Repository for Pediatric Epilepsy. PhD dissertation, Florida International University.

Hamberger, M. J., McClelland, S., r., McKhann, G. M., n., Williams, A. C., Goodman, R. R., 2007. Distribution of auditory and visual naming sites in nonlesional temporal lobe epilepsy patients and patients with space-occupying temporal lobe lesions. Epilepsia 48 (3), 531-8.

Harris, A., 1974. Harris tests of lateral dominance: Manual of directions for administration and interpretation. New York: David McKay Co., Inc.

Holloway, V., Gadian, D., Vargha-Khadem, F., Porter, D., Boyd, S., Connelly, A., 2000. The reorganization of sensorimotor function in children after hemispherectomy: A functional MRI and somatosensory evoked potential study. Brain 123 (12), 2432. 
Huber, P., 1985. Projection pursuit. Annals of statistics 13, 435-475.

Jain, A. K., Duin, R. P., Mao, J., 2000. Statistical pattern recognition: A review. IEEE Transactions on Pattern Analysis and Machine Intelligence 22 (1), 4-37.

Jenkinson, M., Bannister, P., Brady, M., Smith, S., 2002. Improved optimization for the robust and accurate linear registration and motion correction of brain images. Neuroimage 17 (2), 825-41.

Jenkinson, M., Smith, S., 2001. A global optimisation method for robust affine registration of brain images. Med Image Anal 5 (2), 143-56.

Joliffe, I. T., Morgan, B. J., 1992. Principal component analysis and exploratory factor analysis. Stat Methods Med Res 1 (1), 69-95.

Jollife, I., 2002. Principal Component Analysis, 2nd Edition. Springer Series in Statistics. Springer, New York.

Just, M. A., Carpenter, P. A., Keller, T. A., Eddy, W. F., Thulborn, K. R., 1996. Brain activation modulated by sentence comprehension. Science 274 (5284), 114-6.

Khedr, E. M., Hamed, E., Said, A., Basahi, J., 2002. Handedness and language cerebral lateralization. Eur J Appl Physiol 87 (4-5), 469-73.

Knecht, S., Drager, B., Deppe, M., Bobe, L., Lohmann, H., Floel, A., Ringelstein, E. B., Henningsen, H., 2000. Handedness and hemispheric language dominance in healthy humans. Brain 123 Pt 12, 2512-8.

Kurthen, M., Helmstaedter, C., Linke, D. B., Hufnagel, A., Elger, C. E., Schramm, J., 1994. Quantitative and qualitative evaluation of patterns of cerebral language dominance. an amobarbital study. Brain Lang 46 (4), 536-64.

Kwong, K. K., Belliveau, J. W., Chesler, D. A., Goldberg, I. E., Weisskoff, R. M., Poncelet, B. P., Kennedy, D. N., Hoppel, B. E., Cohen, M. S., Turner, R., et al., 1992. Dynamic magnetic resonance imaging of human brain activity during primary sensory stimulation. Proc Natl Acad Sci USA 89 (12), 5675-9.

Lahou, M., Guillen, M., Adjouadi, M., Gaillard, W., 2006. An online web-based repository site of fmri medical images and clinical data for childhood epilepsy. Mednet, pp.120-127.

Liegeois, F., Connelly, A., Cross, J. H., Boyd, S. G., Gadian, D. G., Vargha-Khadem, F., Baldeweg, T., 2004. Language reorganization in children with early-onset lesions of the left hemisphere: an fmri study. Brain 127 (Pt 6), 1229-36. 
Liegeois, F., Cross, J. H., Gadian, D. G., Connelly, A., 2006. Role of fmri in the decisionmaking process: Epilepsy surgery for children. Journal of Magnetic Resonance Imaging 23 (6), 933-940.

Loening, A., Gambhir, S., 2001. AMIDE: A completely free system for medical imaging data analysis. J Nucl Med 42, 827.

Marrelec, G., Ciuciu, P., P'el'egrini-Issac, M., Benali, H., 2004. Estimation of the hemodynamic response in event-related functional MRI: Bayesian networks as a framework for efficient Bayesian modeling and inference. IEEE transactions on medical imaging 23 (8), 959-967.

Mbwana, J., Berl, M. M., Ritzl, E. K., Rosenberger, L., Mayo, J., Weinstein, S., Conry, J. A., Pearl, P. L., Shamim, S., Moore, E. N., Sato, S., Vezina, L. G., Theodore, W. H., Gaillard, W. D., 2009. Limitations to plasticity of language network reorganization in localization related epilepsy. Brain 132 (Pt 2), 347-56.

Medina, L. S., Bernal, B., Dunoyer, C., Cervantes, L., Rodriguez, M., Pacheco, E., Jayakar, P., Morrison, G., J., R., Altman, N. R., 2005. Seizure disorders: functional MR imaging for diagnostic evaluation and surgical treatment-prospective study. Radiology 236 (1), 247-53.

Moeller, J. R., Strother, S. C., Sidtis, J. J., Rottenberg, D. A., 1987. Scaled subprofile model: a statistical approach to the analysis of functional patterns in positron emission tomographic data. J Cereb Blood Flow Metab 7 (5), 649-58.

Muller, R., Rothermel, R., Behen, M., Muzik, O., Mangner, T., Chugani, H., 1998a. Developmental changes of cortical and cerebellar motor control: a clinical positron emission tomography study with children and adults. Journal of child neurology 13 (11), 550 .

Muller, R., Rothermel, R., Muzik, O., Becker, C., Fuerst, D., Behen, M., Mangner, T., Chugani, H., 1998b. Determination of language dominance by [15o]-water pet in children and adolescents: A comparison with the wada test. Journal of Epilepsy 11 (3), $152-161$.

Nielsen, F., Hansen, L., 2000. Experiences with Matlab and VRML in functional neuroimaging visualizations. In: VDE2000-Visualization Development Environments, Workshop Proceedings, Princeton, New Jersey, USA, April. Citeseer, pp. 27-28.

Nolf, E., Voet, T., Jacobs, F., Dierckx, R., Lemahieu, I., XMedCon-An open source medical image conversion toolkit. European Journal of Nuclear Medicine, ISSN, $0340-6997$. 
Ogawa, S., Lee, T. M., Kay, A. R., Tank, D.W., 1990. Brain magnetic resonance imaging with contrast dependent on blood oxygenation. Proc Natl Acad Sci USA 87 (24), 9868-72.

Ojemann, G., Ojemann, J., Lettich, E., Berger, M., 2008. Cortical language localization in left, dominant hemisphere. an electrical stimulation mapping investigation in 117 patients. 1989. J Neurosurg 108 (2), 411-21.

Oldfield, R., 1971. The assessment and analysis of handedness: the Edinburgh inventory. Neuropsychologia 9 (1), 97-113.

Penny, W., Kilner, J., Blankenburg, F., 2007. Robust bayesian general linear models. NeuroImage 36 (3), 661-671.

Petersen, S. E., Fox, P. T., Posner, M. I., Mintun, M., Raichle, M. E., 1988. Positron emission tomographic studies of the cortical anatomy of single-word processing. Nature 331 (6157), 585-9.

Petersson, K. M., Nichols, T. E., Poline, J. B., Holmes, A. P., 1999. Statistical limitations in functional neuroimaging I. non-inferential methods and statistical models.

Philosophical Transactions of the Royal Society B-Biological Sciences 354 (1387), 1239-1260.

Price, C. J., Crinion, J., Friston, K. J., 2006. Design and analysis of fmri studies with neurologically impaired patients. J Magn Reson Imaging 23 (6), 816-26.

Price, C. J., Devlin, J. T., Moore, C. J., Morton, C., Laird, A. R., 2005. Meta-analyses of object naming: effect of baseline. Hum Brain Mapp 25 (1), 70-82.

Pujol, J., Deus, J., Losilla, J. M., Capdevila, A., 1999. Cerebral lateralization of language in normal left-handed people studied by functional mri. Neurology 52 (5), 1038-43.

Rabin, M., Narayan, V., Kimberg, D., Casasanto, D., Glosser, G., Tracy, J., French, J., Sperling, M., Detre, J., 2004. Functional mri predicts post-surgical memory following temporal lobectomy. Brain 127 (10), 2286.

Ramsey, N. F., Sommer, I. E., Rutten, G. J., Kahn, R. S., 2001. Combined analysis of language tasks in fMRI improves assessment of hemispheric dominance for language functions in individual subjects. Neuroimage 13 (4), 719-33.

Rasmussen, T., Milner, B., 1977. The role of early left-brain injury in determining lateralization of cerebral speech functions. Ann N Y Acad Sci 299, 355-69.

Reale, R. A., Calvert, G. A., Thesen, T., Jenison, R. L., Kawasaki, H., Oya, H., Howard, M. A., Brugge, J. F., 2007. Auditory-visual processing represented in the human superior temporal gyrus. Neuroscience 145 (1), 162-84. 
Richardson, M., Strange, B., Thompson, P., Baxendale, S., Duncan, J., Dolan, R., 2004. Pre-operative verbal memory fmri predicts post-operative memory decline after left temporal lobe resection. Brain 127 (11), 2419.

Risse, G. L., Gates, J. R., Fangman, M. C., 1997. A reconsideration of bilateral language representation based on the intracarotid amobarbital procedure. Brain Cogn 33 (1), $118-32$.

Rorden, C., 2004. MRIcro. Computer software]. Retrieved May 14, 2003, from http://people. cas. sc. edu/rorden.

Rorden, C., Brett, M., 2000. Stereotaxic display of brain lesions. Behavioural Neurology 12 (191-200), 191-200.

Rosenberger, L. R., Zeck, J., Berl, M. M., Moore, E. N., Ritzl, E. K., Shamim, S., Weinstein, S. L., Conry, J. A., Pearl, P. L., Sato, S., Vezina, L. G., Theodore, W. H., Gaillard, W. D., 2009. Interhemispheric and intrahemispheric language reorganization in complex partial epilepsy. Neurology 72 (21), 1830-6.

Rowe, D. B., Hoffmann, R. G., 2006. Multivariate statistical analysis in fmri. IEEE Eng Med Biol Mag 25 (2), 60-4. Samanwoy, G., Hojjat, A., 2008. Principal component analysis-enhanced cosine radial basis function neural network for robust epilepsy and seizure detection. IEEE Transactions On Biomedical Engineering 55 (2), 512-518.

Seghier, M., Lazeyras, F., Pegna, A., Annoni, J., Khateb, A., 2008. Group analysis and the subject factor in functional magnetic resonance imaging: analysis of fifty righthanded healthy subjects in a semantic language task. Human Brain Mapping 29 (4).

Smith, J. F., Chen, K. W., Johnson, S., Morrone-Strupinsky, J., Reiman, E. M., Nelson, A., Moeller, J. R., Alexander, G. E., 2006. Network analysis of single-subject fMRI during a finger opposition task. Neuroimage 32 (1), 325-332.

Smith, S. M., Jenkinson, M., Woolrich, M. W., Beckmann, C. F., Behrens, T. E., Johansen-Berg, H., Bannister, P. R., De Luca, M., Drobnjak, I., Flitney, D. E., Niazy, R. K., Saunders, J., Vickers, J., Zhang, Y., De Stefano, N., Brady, J. M., Matthews, P. M., 2004. Advances in functional and structural $\mathrm{mr}$ image analysis and implementation as fsl. Neuroimage 23 Suppl 1, S208-19.

Spreer, J., Arnold, S., Quiske, A., Wohlfarth, R., Ziyeh, S., Altenmuller, D., Herpers, M., Kassubek, J., Klisch, J., Steinhoff, B. J., Honegger, J., Schulze-Bonhage, A., Schumacher, M., 2002. Determination of hemisphere dominance for language: comparison of frontal and temporal fmri activation with intracarotid amytal testing. Neuroradiology 44 (6), 467-74. 
Springer, J. A., Binder, J. R., Hammeke, T. A., Swanson, S. J., Frost, J. A., Bellgowan, P. S., Brewer, C. C., Perry, H. M., Morris, G. L., Mueller,W. M., 1999. Language dominance in neurologically normal and epilepsy subjects: a functional mri study. Brain 122 (Pt 11), 2033-46.

Stanberry, L., Nandy, R., Cordes, D., 2003. Cluster analysis of fMRI data using dendrogram sharpening. Human brain mapping 20 (4), 201-219.

Staudt, M., Grodd, W., Niemann, G., Wildgruber, D., Erb, M., Krageloh-Mann, I., 2001. Early left periventricular brain lesions induce right hemispheric organization of speech. Neurology 57 (1), 122-5.

Staudt, M., Lidzba, K., Grodd, W., Wildgruber, D., Erb, M., Krageloh-Mann, I., 2002. Right-hemispheric organization of language following early left-sided brain lesions: functional MRI topography. Neuroimage 16 (4), 954-67.

Stoodley, C. J., Schmahmann, J. D., 2009. Functional topography in the human cerebellum: a meta-analysis of neuroimaging studies. Neuroimage 44 (2), 489-501.

Sugiura, M., Watanabe, J., Maeda, Y., Matsue, Y., Fukuda, H., Kawashima, R., 2004. Different roles of the frontal and parietal regions in memory-guided saccade: a pca approach on time course of bold signal changes. Hum Brain Mapp 23 (3), 129-39.

Sui, J., Adali, T., Pearlson, G., Calhoun, V., 2009. An ica-based method for the identification of optimal fmri features and components using combined groupdiscriminative techniques. Neuroimage 46 (1), 73-86.

Suma, H., Murali, S., 2007. Principal component analysis and classification of fmri activation maps. International Journal of Computer and Network Security 7 (11), 235242.

Szaflarski, J. P., Binder, J. R., Possing, E. T., McKiernan, K. A., Ward, B. D., Hammeke, T. A., 2002. Language lateralization in left-handed and ambidextrous people: fMRI data. Neurology 59 (2), 238-44.

Szaflarski, J. P., Schmithorst, V. J., Altaye, M., Byars, A. W., Ret, J., Plante, E., Holland, S. K., 2006. A longitudinal functional magnetic resonance imaging study of language development in children 5 to 11 years old. Ann Neurol 59 (5), 796-807.

Thivard, L., Hombrouck, J., Tezenas du Montcel, S., Delmaire, C., Cohen, L., Samson, S., Dupont, S., Chiras, J., Baulac, M., Lehericy, S., 2005. Productive and perceptive language reorganization in temporal lobe epilepsy. Neuroimage 24 (3), 841-851.

Tito, M., Cabrerizo, M., Ayala, M., Barreto, A., Miller, I., Jayakar, P., Adjouadi, M., 2009. Classification of electroencephalographic seizure recordings into ictal and interictal files using correlation sum. Computers in Biology and Medicine 39 (7), 604-614. 
Tou, J. T., Gonzalez, R. C., 1974. Pattern Recognition Principles. Applied Mathematics and Computation, Reading, Mass.: Addison-Wesley.

Turk, M., Pentland, A., 1991. Eigenfaces for recognition. J. Cognitive Neuroscience 3 (1), $71-86$.

Turkeltaub, P., Flowers, D., Verbalis, A., Miranda, M., Gareau, L., Eden, G., 2004. The Neural Basis of Hyperlexic Reading An fMRI Case Study. Neuron 41 (1), 11-25.

Turkeltaub, P., Gareau, L., Flowers, D., Zeffiro, T., Eden, G., 2003. Development of neural mechanisms for reading. Nature Neuroscience 6 (7), 767-773.

Turner, R., Rees, G., Howseman, A., Josephs, O., Frith, C., Friston, K., Frackowiak, R., 1997. Characterising the relationship between bold contrast and regional cerebral blood flow measurements by varying the stimulus presentation rate. Neuroimage 6 , 270-278.

Vaidya, C., Kaplan, L., Kenworthy, L., Gilotty, L., Girton, L., Fos, J., Lacey, E., Gaillard, W., 2005. Altered neural basis of controlling interference from social and non-social information in childhood autism: A functional magnetic resonance study. Journal of Cognitive Neuroscience, 222-222.

Viera, A. J., Garrett, J. M., 2005. Understanding inter-observer agreement: the kappa statistic. Fam Med 37 (5), 360-3.

Viviani, R., Gron, G., Spitzer, M., 2005. Functional principal component analysis of fmri data. Hum Brain Mapp 24 (2), 109-29.

Voets, N. L., Adcock, J. E., Flitney, D. E., Behrens, T. E., Hart, Y., Stacey, R., Carpenter, K., Matthews, P. M., 2006. Distinct right frontal lobe activation in language processing following left hemisphere injury. Brain 129 (Pt 3), 754-66.

Weber, B., Wellmer, J., Reuber, M., Mormann, F., Weis, S., Urbach, H., Ruhlmann, J., Elger, C., Fernandez, G., 2006. Left hippocampal pathology is associated with atypical language lateralization in patients with focal epilepsy. Brain 129 (2), 346.

Weber, D., Arnaud, D., Hough, M., Oo-stenveld, R., Dalal, S., 2004. EEG and MRI Matlab toolbox. Internet http://eeg. sourceforge. net 1.

Weder, B., Schindler, K., Loher, T., Wiest, R., Wissmeyer, M., Ritter, P., Lovblad, K., Donati, F., Missimer, J., 2006. Brain areas involved in medical temporal lobe seizures. Human Brain Mapping 27, 520-534.

Wilke, M., Lidzba, K., 2007. Li-tool: a new toolbox to assess lateralization in functional mr-data. J Neurosci Methods 163 (1), 128-36. 
Wilke, M., Schmithorst, V. J., 2006. A combined bootstrap/histogram analysis approach for computing a lateralization index from neuroimaging data. Neuroimage 33 (2), $522-30$.

Wise, R., Chollet, F., Hadar, U., Friston, K., Hoffner, E., Frackowiak, R., 1991. Distribution of cortical neural networks involved in word comprehension and word retrieval. Brain 114 (Pt 4), 1803-17.

Woermann, F. G., Jokeit, H., Luerding, R., Freitag, H., Schulz, R., Guertler, S., Okujava, M., Wolf, P., Tuxhorn, I., Ebner, A., 2003. Language lateralization by wada test and fmri in 100 patients with epilepsy. Neurology 61 (5), 699-701.

Woods, R. P., Dodrill, C. B., Ojemann, G. A., 1988. Brain injury, handedness, and speech lateralization in a series of amobarbital studies. Ann Neurol 23 (5), 510-8.

Woolrich, M. W., Ripley, B. D., Brady, M., Smith, S. M., 2001. Temporal autocorrelation in univariate linear modeling of fmri data. Neuroimage 14 (6), 137086.

Worsley, K., Evans, A., Marrett, S., Neelin, P., 1992. A three-dimensional statistical analysis for CBF activation studies in human brain. Journal of Cerebral Blood Flow and Metabolism 12, 900-900.

Wyllie, E., Naugle, R., Chelune, G., Luders, H., Morris, H., Skibinski, C., 1991. Intracarotid amobarbital procedure: Ii. lateralizing value in evaluation for temporal lobectomy. Epilepsia 32 (6), 865-9.

Yetkin, F. Z., Swanson, S., Fischer, M., Akansel, G., Morris, G., Mueller, W., Haughton, V., 1998. Functional $\mathrm{mr}$ of frontal lobe activation: comparison with wada language results. AJNR Am J Neuroradiol 19 (6), 1095-8.

Yoo, S., Fairneny, T., Chen, N., Choo, S., Panych, L., Park, H., Lee, S., Jolesz, F., 2004. Brain-computer interface using fMRI: spatial navigation by thoughts. Neuroreport 15 (10), 1591.

You, X., Guillen, M., Bernal, B., Gaillard, W. D., Adjouadi, M., 2009. fmri activation pattern recognition: A novel application of pca in language network of pediatric localization related epilepsy, 5397-5400.

Yuan, W., Szaflarski, J. P., Schmithorst, V. J., Schapiro, M., Byars, A. W., Strawsburg, R. H., Holland, S. K., 2006. fmri shows atypical language lateralization in pediatric epilepsy patients. Epilepsia 47 (3), 593-600. 
VITA

\section{XIAOZHEN YOU}

February 26, 1982 Born, Fujian, China

2001-2005 B.S., Biomedical Engineering Shanghai

Jiao Tong University Shanghai, China

2005-2010 Doctorate Candidate in Biomedical Engineering

Florida International University

Miami, Florida

\section{PUBLICATIONS AND PRESENTATIONS}

1. You X, Adjouadi M, Guillen M, Ayala M, Barreto A, Rishe N, Sullivan J, Dlugos D, VanMeter J, Morris D, Donner E, Bjornson B, Smith M, Bernal B, Berl M, Gaillard WD. (2010): Sub-Patterns of language network reorganization in Pediatric Localization Related Epilepsy- a Multisite Study, in press by Human Brain Mapping 2010.

2. You X, Yao Z. (2005): Application of XML in DICOM, Proceedings of SPIE International Symposium Medical Imaging, Vol. 5748 No.57, (2005)

3. You X, Teng N, Ayala M, Wang L, Barreto A, Rishe N, Adjouadi M. (2007): A Practical Study on Autism Detection from EEG using Artificial Neural Networks, Proc. of the IASTED International Conference on Graphics and Visualization in Engineering (GVE 2007), January 3-5, 2007, Clearwater, Florida, USA, ISBN: 9780-88986-625-6, pp. 41-46.

4. You X, Guillen MR, Gaillard WD, Adjouadi M. (2009): Application of Nonlinear Classifiers with the Principal Component Analysis in fMRI Language Activation Pattern Recognition in a Multisite Study for Pediatric Epilepsy. International Conference on Image Processing, Computer Vision, and Pattern Recognition IPCV, July 13-16, 2009, Las Vegas, USA, ISBN:1-60132-119-8, pp. 754-758.

5. You X, Guillen MR, Bernal B, Gaillard WD, Adjouadi M. (2009): fMRI activation pattern recognition: A novel application of PCA in Language Network of Pediatric Localization Related Epilepsy. 31ST Annual International Conference of the IEEE Engineering in Medicine and Biology Society: September 2-6, 2009, Minneapolis, MN. Conf Proc IEEE Eng Med Biol Soc. 1:5397-5400.

6. You X, Adjouadi M, Guillen MR, Bernal B, Ayala M, Barreto A, Rishe N, Gaillard WD. (2008):Automatic Decision Making Process For Classification of fMRI Patterns Integrating the Principal Component Analysis and the Laterality Index in Pediatric Epilepsy. Proceedings 30th Annual Meeting of the Society for Medical Decision Making SMDM, Philadelphia, Pennsylvania, pp. 154, Oct.19-22, 2008. 
7. You X, Guillen M, Adjouadi M, "The Merit Of Principal Component Analysis In fMRI Language Pattern Recognition For Pediatric Epilepsy", International Journal of Medical Implants and Devices, Medical and Engineering Pub, Inc., Vol. 4 (1), pp. 25, 2009.

8. Adjouadi M, You XZ, Guillen M, Ayala M, Cabrerizo M, Jayakar P, Barreto A, Rishe N, Sullivan J, Dlugos D, Berl M, VanMeter J., Morris D, Donner E, Bjornson B., Smith M., Bernal B., Gaillard WD, "Integrating Nonlinear Decision Functions with Principal Component Analysis in fMRI Language Activation Patterns Classification". EPILEPSIA, Vol. 50, pp. 212-213, Suppl. 11, Nov 2009.

9. Gaillard W.D., You X, Bernal B, Guillen MR, Ayala M, Jayakar P, Barreto A, Rishe N, Sullivan J, Dlugos D, Berl MM, VanMeter J, Morris D, Donner E, Bjornson B, Smith M. Adjouadi M, "Pediatric Functional Imaging Consortium: Sub-Patterns of Language Dominance in Pediatric Localization Related Epilepsy Identified by Data Driven Separation Analysis", EPILEPSIA, Vol. 50, pp. 437-437, Suppl. 11, Nov 2009.

10. Guillen M, Adjouadi M, Bernal B, You X, Barreto A, Rishe N, Jayakar P, Gaillard WD , "Functional Magnetic Resonance Imaging Group Decision Making Based on Brain Asymmetry", EPILEPSIA, Vol. 50, pp. 210-211, Suppl. 11, Nov 2009.

11. Goryawala M, Guillen M, You X, Adjouadi M, "A New Algorithm as an Extension to the Gradient Descent Method for Functional Brain Activation Classification", International Journal of Medical Implants and Devices, Medical and Engineering Pub, Inc., Vol. 4 (1), pp. 25, 2009.

12. Guillen M, Adjouadi M, You X, Goryawala M, "Application of Nonlinear Decision Functions in the Identification of Language Dominance Behavior", International Journal of Medical Implants and Devices, Medical and Engineering Pub, Inc., Vol. 4 (1), pp. 26, 2009.

13. Guillen MR, Adjouadi M, Ayala M, You X, Barreto A, Rishe N, Bernal B, Gaillard WD.(2009): Toward fMRI Group Identification based on Brain Lateralization. The IEEE 23rd International Conference on Advanced Information Networking and Applications (AINA-09) University of Bradford, Bradford, UK, May 26-29, 2009, pp. 1025-1030.

14. Guillen MR, Adjouadi M, Bernal B, Ayala M, Barreto A, Rishe N, Lizarraga G, You X, Gaillard WD.(2009): A Knowledge-Based Database System for Visual Rating of fMRI Activation Pattern for Brain Language Network. Tapia Conference 2009: Celebration of Diversity in Computing, Portland, Oregon (ACM press), pp. 1-6.

15. Guillen MR, Adjouadi M, Ayala M, You X, Gaillard WD. (2008): A Unified Approach to fMRI study on Group Identification based on Brain Lateralization. The 6th International Conference on Computing, Communications and Control Technologies. Orlando, Florida, USA. pp. 87-92. 